\title{
Square Beam Antenna Using A Microstrip Patch Array For Stadium Applications
}

\author{
by \\ Erik R. Willis, B.Eng

\begin{abstract}
A thesis submitted to the
Faculty of Graduate and Postdoctoral Affairs

in partial fulfillment of the requirements for the degree of
\end{abstract}

Master of Applied Science in Electrical Engineering

Ottawa-Carleton Institute for Electrical and Computer Engineering

Department of Department of Electronics

Carleton University

Ottawa, Ontario

December, 2015 


\section{(C) Copyright}

Erik R. Willis, 2015 
The undersigned hereby recommends to the

Faculty of Graduate and Postdoctoral Affairs acceptance of the thesis

\title{
Square Beam Antenna Using A Microstrip Patch Array For Stadium Applications
}

\author{
submitted by Erik R. Willis, B.Eng \\ in partial fulfillment of the requirements for the degree of
}

Master of Applied Science in Electrical Engineering

Professor James S. Wight, Thesis Supervisor

Professor Nial Tait, Chair, Department of Electrical Engineering

Ottawa-Carleton Institute for Electrical and Computer Engineering Department of Department of Electronics

Carleton University

December, 2015 


\section{Abstract}

In a Stadium installation of Wi-Fi, ideally a single access point could be used to illuminate a section with little interference from adjacent sections. This can be done with the use of a square beam as opposed to the typical elliptical beam. This thesis explores the creation of a square beam through the use of the Fourier transform. A sinc function in the near-field creates a square beam in the far-field. A 9x9 array with the weighting of the elements being a sinc function produced a square beam in the far-field. The size of the array was reduced by removing low power elements eventually creating a sparse array. The sparse array allowed space for a sub-array to be placed in order to reactively cancel out sidelobes. This reduced sidelobe levels by $12 \mathrm{~dB}$.

A feed network was designed to provide the correct power and phases to the elements without the use of amplifiers or phase shifters. While this low-cost technique is often time consuming, the methodology used in the design of this feed network

provides guidance for future feed network designs. A number of equal and unequal split Wilkinson power dividers were also a focus of this design. A method to quickly address poor return loss was explored because reflections are additive in an array. The method facilitates determining additional lengths of microstrip line without the need to simulate the full array. While the results of this prototype did not meet all specification, it does however, provide a framework to further explore. 


\section{Acknowledgments}

I would like to thank my supervisor Jim Wight. With your support and guidance, I have thoroughly enjoyed this experience. Though there were ups and downs, you were always there to steer me in the right direction. I would also like to thank the staff of the Department of Electronics at Carleton University. You were always able to help when I needed it. I am also grateful for the guidance and support of Roland Smith (Ericsson) and Peter Frank (Petra Microwave). Without you, I'm sure I would have spent countless hours trying to figure out small problems on my own. I also want to thank all my friends and family for the tremendous support throughout my 8 years at Carleton.

The financial support of Carleton University and Ericsson Canada has been very beneficial in helping me realize the prototype presented in this thesis.

Finally, I want to thank my fiance Ali Piwowar for all her support - especially in the final weeks of thesis work. Without you this wouldn't be possible. 


\section{Table of Contents}

Abstract iv

Acknowledgments $\quad$ v

Table of Contents $\quad$ vi

List of Tables $\quad$ ix

List of Figures $\quad \mathrm{x}$

1 Introduction 1

1.1 Motivation . . . . . . . . . . . . . . . . . . . . 1

1.2 Thesis Objectives . . . . . . . . . . . . . . . . . . 2

1.3 Thesis Contributions . . . . . . . . . . . . . . . . 2

1.4 Thesis Organization . . . . . . . . . . . . . . . . . . . 2

2 Background Information $\quad 4$

2.1 Introduction . . . . . . . . . . . . . . . . . 4

2.2 Microstrip Patches . . . . . . . . . . . . . . . . 4

2.3 Antenna Characteristics . . . . . . . . . . . . . 5

2.4 Array Theory . . . . . . . . . . . . . . . . . . . . . . 9

2.5 Fourier Transform . . . . . . . . . . . . . . . . . . 10 
2.6 Duality with Digital Signal Processing Theory . . . . . . . . . . . . . 10

2.7 Squareness . . . . . . . . . . . . . . . . . . . . 12

2.8 Feed Network . . . . . . . . . . . . . . . . . . . . . 13

2.8.1 Power Dividers . . . . . . . . . . . . . . . . . . 15

2.9 Recent Research . . . . . . . . . . . . . . . . . . 26

2.9.1 Flat-Top Radiation Patterns . . . . . . . . . . . . . . 26

2.9.2 Sidelobe Levels . . . . . . . . . . . . . . . . . . . . . . . 27

3 Array Factor Design $\quad 28$

3.1 Introduction . . . . . . . . . . . . . . . . . . 28

3.2 Simulation Tools . . . . . . . . . . . . . . . . . . . . . . . 29

3.3 Square Patch Array . . . . . . . . . . . . . . . . . . . . 29

3.4 Array Reduction . . . . . . . . . . . . . . . . . . 35

3.5 Gaussian Weighted Sampled Sinc Function . . . . . . . . . . . . 43

3.6 Sidelobe Suppression . . . . . . . . . . . . . . . . . . . . . . 49

3.7 Ultra Low Sidelobe . . . . . . . . . . . . . . . . . . 56

3.8 Summary of Results . . . . . . . . . . . . . . . . . 60

3.9 Conclusions . . . . . . . . . . . . . . . . . . . 60

4 Feed Network and System Design $\quad 61$

4.1 Introduction . . . . . . . . . . . . . . . . . 61

4.2 Patch Design ........................ 61

4.3 Feed Network Design . . . . . . . . . . . . . . . . 65

4.4 Power Dividers . . . . . . . . . . . . . . . . . . . . 71

4.4.1 Switch to modified Wilkinson . . . . . . . . . . . . . 77

4.5 Tuning Stubs . . . . . . . . . . . . . . . . 78

4.6 Return Loss Methodology . . . . . . . . . . . . . . . . . . . . 80 
4.7 Radiation Pattern . . . . . . . . . . . . . . . . . 82

4.8 Conclusions . . . . . . . . . . . . . . . . . . 86

$\begin{array}{llr}5 & \text { Testing Results } & 88\end{array}$

5.1 Introduction . . . . . . . . . . . . . . . . 88

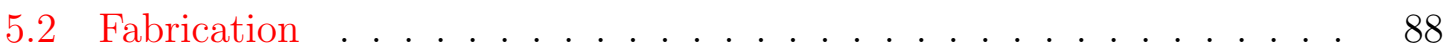

5.3 Test Methodology . . . . . . . . . . . . . . . . . . . . 92

5.4 Component Results . . . . . . . . . . . . . . . . . . . 93

6 Conclusions 102

6.1 Future Work . . . . . . . . . . . . . . . . 106

$\begin{array}{ll}\text { List of References } & 107\end{array}$

$\begin{array}{ll}\text { Appendix A Matlab Code } & 110\end{array}$

$\begin{array}{lll}\text { Appendix B Empire Setup } & 112\end{array}$ 


\section{List of Tables}

2.1 Duality Between Signal Processing and Array Design [1]. . . . . . . . 11

3.1 Summary of Results. . . . . . . . . . . . . . . . . . . . . . 60

4.1 Weighting of patches. . . . . . . . . . . . . . 67

4.2 Power splitter impedances. . . . . . . . . . . . . . . . . . . 72

4.3 Divider A Dimensions. . . . . . . . . . . . . . . 73

4.4 Divider B Dimensions. . . . . . . . . . . . . . . . . . . 74

4.5 Divider C Dimensions. . . . . . . . . . . . . . . . . . . 74

4.6 Divider D Dimensions. . . . . . . . . . . . . . . . . . . 74

4.7 Simulated scattering parameters of dividers. . . . . . . . . . . 76

4.8 Comparison between ideal, feed network alone, and full system. . . . 84

5.1 Measured phases of cables. . . . . . . . . . . . . . . . . . . 93

5.2 Measured scattering parameters of dividers. . . . . . . . . . . . . . 94

5.3 Measured scattering parameters of three-way dividers. . . . . . . . . 95

5.4 Measured scattering parameters of feed network sections. . . . . . . . 97

5.5 Results of full antenna configurations. . . . . . . . . . . . . . . 100

6.1 Target Specifications vs Measured Values. . . . . . . . . . . . . 105 


\section{List of Figures}

2.1 Antenna Radiation Pattern Characteristics [2]. . . . . . . . . 6

2.2 Square radiation pattern from bore sight. . . . . . . . . . . . . 12

2.3 Different Layouts for Lumped Matching Techniques [3] . . . . . . . . 14

2.4 Two-way lossless power divider $[3] . \ldots \ldots \ldots \ldots$

2.5 Two-way resistive power divider [3]. . . . . . . . . . . . 17

2.6 Simplified two port Wilkinson power divider [3] . . . . . . . . . 18

2.7 Two-way Wilkinson power divider [3] . . . . . . . . . . . . . . . 19

2.8 Even mode analysis of Wilkinson power divider [3] . . . . . . . . . 19

2.9 Odd mode analysis of Wilkinson power divider [3]. . . . . . . . . . 21

2.10 Unequal split wilkinson power divider. . . . . . . . . . . . . . 22

2.11 Wilkinson power divider port 2 and $3[4] \ldots \ldots \ldots \ldots$

3.1 Level of squareness for 4 different sized arrays. . . . . . . . . . . 30

3.2 3D Plot of $9 \times 9$ Array. . . . . . . . . . . . . . . . . . . 31

3.3 Sidelobe level for 4 different sized arrays. . . . . . . . . . . . . . . 32

3.4 Layout of $9 \mathrm{x} 9$ array with weighting. . . . . . . . . . . 33

3.5 Radiation pattern of $9 \mathrm{x} 9$ array with sinc weighting. . . . . . . 34

3.6 Layout after removing low power elements. . . . . . . . . . 35

3.7 Radiation pattern after removing low power elements. . . . . . . . 36

3.8 Layout with uniform weighting in center. . . . . . . . . . 37 
3.9 Layout with uniform weighting in cross elements. . . . . . . . . . . 38

3.10 Radiation Pattern with uniform weighting in cross elements. . . . . . 39

3.11 Sinc function overlaid over layout of cross. . . . . . . . . . . . . . . . 40

3.12 Layout with uniform weighting in a sinc pattern. . . . . . . . . . . . 41

3.13 Radiation Pattern with uniform weighting in a sinc layout. . . . . . . 42

3.14 Weighting used for Gaussian distribution. . . . . . . . . . . . . . 44

3.15 Comparison of different weightings of squaring elements. . . . . . . . 45

3.16 Radiation pattern of a Gaussian distribution over sinc layout. . . . . 46

3.17 Location of the port feeding the patches. . . . . . . . . . . . . 47

3.18 Radiation pattern with the rotated feed. . . . . . . . . . . . 47

3.19 Radiation pattern with varying element spacing. . . . . . . . . . 48

3.20 Radiation pattern with phase of the beam forming array. . . . . . . . 50

3.21 Radiation pattern with phase of the sidelobe supressing sub array. . . 51

3.22 Radiation pattern of the sidelobe supressing sub array at different positions. . . . . . . . . . . . . . . . . . 52

3.23 Radiation pattern of the full array with suppressors at different positions. 53

3.24 Radiation pattern of the full array with various weightings. . . . . . . 54

3.25 Dimensions of full array. . . . . . . . . . . . . . . . . 55

3.26 Radiation pattern of full array. . . . . . . . . . . . . . 56

3.27 Layout of gaussian distributed array. . . . . . . . . . . . . 57

3.28 Comparison between Gaussian distributed with and without suppressing sub-array. . . . . . . . . . . . . . . . . 58

3.29 Comparison between optimized position and non-optimized. . . . . . 59

4.1 Layout of the microstrip patch. . . . . . . . . . . . . . . 62

4.2 Stack up of array. . . . . . . . . . . . . . . . . 63

4.3 Difference between single feed and push/pull feed. . . . . . . . . . . . 64 
4.4 Comparison of slot fed patch and probed patch from Section 3. . . . 65

4.5 Symmetry in patch layout. . . . . . . . . . . . . 66

4.6 Section of the feed network designed in ADS. . . . . . . . . . . . 69

4.7 Full layout of feed network. . . . . . . . . . . . . . . . . . . 71

4.8 Layout of power dividers. . . . . . . . . . . . . . . . 73

4.9 ADS Layout for Modified Wilkinson. . . . . . . . . . . . . . 75

4.10 Reflection Coefficient of Modified Wilkinson. . . . . . . . . . . . 76

4.11 Radiation pattern with a T-Junction Divider. . . . . . . . . . . 77

4.12 Radiation pattern with a Modified Wilkinson Divider. . . . . . . . . 78

4.13 Return loss of the patches with tuning stubs. . . . . . . . . . . 79

4.14 Schematic used to determine optimal length of divider arms. . . . . . 81

4.15 Radiation pattern of the full system in Azimuth and Elevation. . . . . 82

4.16 Layout of port field probes. . . . . . . . . . . . . . . . . 83

4.17 Modified microstrip line lengths effects on radiation pattern. . . . . . 85

4.18 Comparison between port fed array and feed network array . . . . . . 87

5.1 Effects on squareness with different phase offsets. . . . . . . . . . . . 90

5.2 Photo of array inside the anechoic chamber. . . . . . . . . . . . . . 91

5.3 Photo of the feed network. . . . . . . . . . . . . . . . 91

5.4 Setup of the radiation pattern testing. . . . . . . . . . . . . . . 92

5.5 Layout of two-way dividers. . . . . . . . . . . . . . . . . . . 94

5.6 Layout of three-way dividers. . . . . . . . . . . . . . . . 95

5.7 Layout of different sections of feed network. . . . . . . . . . . 96

5.8 Layout of different sections of feed network. . . . . . . . . . . . 97

5.9 Radiation pattern of array with 344 thou air gap. . . . . . . . . . . . 98

5.10 Radiation pattern of array with 250 thou air gap. . . . . . . . . . . . 99

5.11 Radiation pattern of Gaussian array with 344 thou air gap. . . . . . . 100 
6.1 Radiation pattern with relevant specifications labeled. . . . . . . . . . 104

6.2 Radiation pattern of measured and simulated array. . . . . . . . . . 105

B.1 Structure setup in Empire XPU. . . . . . . . . . . . . . . . . . . . . 112

B.2 Main work screen in Empire XPU. . . . . . . . . . . . . . . . . . . . 113

B.3 Simulation Set up in Empire XPU. . . . . . . . . . . . . . . . 114

B.4 Port set up in Empire XPU. . . . . . . . . . . . . . . . . 115 


\section{Chapter 1}

\section{Introduction}

\subsection{Motivation}

As more and more devices become connected, the need to improve Wi-Fi connections increases. Challenges arise where many devices are concentrated in a relatively small area, for example a stadium. There are a number of challenges in regards to setting up a stadium with Wi-Fi. A large number of access points are required to provide coverage to all sections. This is due to using a narrow beam width to reduce interference with adjacent access points. Another problem occures in the need to fully illuminate rectangular shaped sections with elliptical beam, as dead spots towards the top corners are unavoidable. While it is possible to install another access point directed in that area, this overlap will cause interference between adjacent channels.

This thesis confronts these two issues in the design of a microstrip patch array with a square beam and low sidelobes. This solution reduces the number of access points required and provides superior coverage to current antenna solutions with reduced interference. In addition to improved performance, the square beam significantly reduces costs due to fewer access points being required. 


\subsection{Thesis Objectives}

Microstrip arrays have been used to reduce sidelobes with a number of different weightings and configuration. None however look at forming a square beam while maintaining low sidelobes. This thesis aims to address the design process of a square beam microstrip patch array with low sidelobe performance.

The antenna was designed to operate within the $2.4 \mathrm{GHz}$ band and has the ability to be scaled to the $5 \mathrm{GHz}$ band if required. The sidelobe goal was to be at or below $-30 \mathrm{~dB}$ as well as having a square beam.

\subsection{Thesis Contributions}

This thesis contributes to knowledge in antenna designs through the following:

- A Design of square beam antenna patterns in microstrip patch arrays.

- A Methodology of creating an unequal weighted sparse array.

- A reactive method of sidelobe reduction in patch arrays.

- A method for the reduction of return loss with reduced simulation time.

\subsection{Thesis Organization}

This thesis is divided into 6 chapters as follows:

Chapter 1 contains the motivation, objectives and contributions of the thesis.

Chapter 2 provides background information on microstrip patches, the near-field to far-field transformation, feed networks, and power dividers. 
Chapter 3 investigates a method used to create a square beam from a sparse array. It also explores a method of sidelobe suppression. Simulation results of a port fed array are in this chapter.

Chapter 4 describes the design process of a feed network with unequal Wilkinson power dividers. The methodology of reducing return loss is also presented. Simulation results for the full feed network fed array are included in this chapter.

Chapter 5 includes the fabrication and testing process of the array. Results from the testing is tabulated here.

Chapter 6 presents a summary of the work done and a comparison between simulated and measured data, as well as opportunity for future work. 


\section{Chapter 2}

\section{Background Information}

\section{$2.1 \quad$ Introduction}

This chapter will provide information on array theory, Fourier transforms, feed networks, and power dividers. The first two sections provide the framework of design, and the second two sections are required to bring the design from a simulated product to a manufactured one.

\subsection{Microstrip Patches}

Microstrip patch antennas consist of a layer of conductor over a dielectric substrate, with a ground plane on the opposite side. There are a number of methods of feeding a microstrip patch, so this section focuses on the slot-fed, aperture coupled method. A slot is cut into the ground plane and a second layer of substrate is required on the bottom of the ground plane, with a feed plane, on the bottom. The feed strip is often made of the same conductor as the patch layer, and runs past the slot on the layer above it [5].

The slots in the ground plane allow energy to travel through and couple onto the 
patch above it. In this thesis, this patch is actually a sub patch that radiates through a small air gap to illuminate a larger patch on a different substrate. This is done to increase bandwidth [6].

\subsection{Antenna Characteristics}

Most antenna characteristics are defined from the far-field region [7]. This is a region defined by:

$$
r \geq \frac{\left(2 L^{2}\right)}{\lambda}
$$

Where $r$ is the distance away from the antenna, $L$ is the maximum linear dimension

of the antenna, and $\lambda$ is the wavelength of the frequency of operation. Anything beyond this region is known as the far-field. In the far-field, the waves being radiated from the antenna resemble plane waves and are represented by:

$$
\begin{gathered}
\mathbf{E}_{s}=-\eta_{0} \mathbf{a}_{r} \times \mathbf{H}_{s} \\
\mathbf{H}_{s}=\frac{1}{\eta_{0}} \mathbf{a}_{r} \times \mathbf{E}_{s}
\end{gathered}
$$

$\eta_{o}$ is the free space impedance of $120 \pi$. Using Poynting's theorem the time average power density vector is calculated.

$$
\mathbf{P}(r, \theta, \phi)=\frac{1}{2} \operatorname{Re}\left[\mathbf{E}_{s} \times \mathbf{H}_{s}^{*}\right]
$$

The total power radiated as a function of $\mathrm{r}, \theta$, and $\phi$ are found by integrating the 
Poynting vector over a closed spherical surface. The radiation intensity is the expression of power radiated per unit solid angle.

$$
P_{\text {rad }}=\iint P(r, \theta, \phi) r^{2} \sin \theta d \theta d \phi
$$

There are two principal pattern cuts for most antenna radiation patterns. These are the patterns in the planes containing the principle $\mathrm{E}$ and $\mathrm{H}$ plane. The E-plane contains the electric field vector and the H-plane contains the magnetic field vector; they are always orthogonal to each other [2]. These planes are typically defined as $\phi=0^{\circ}$ or $\phi=90^{\circ}$.

The definition of half power beamwidth by IEEE is:

"In a plane containing the direction of the maximum of a beam, the angle between the two directions in which the radiation intensity is one-half value of the beam." [8]

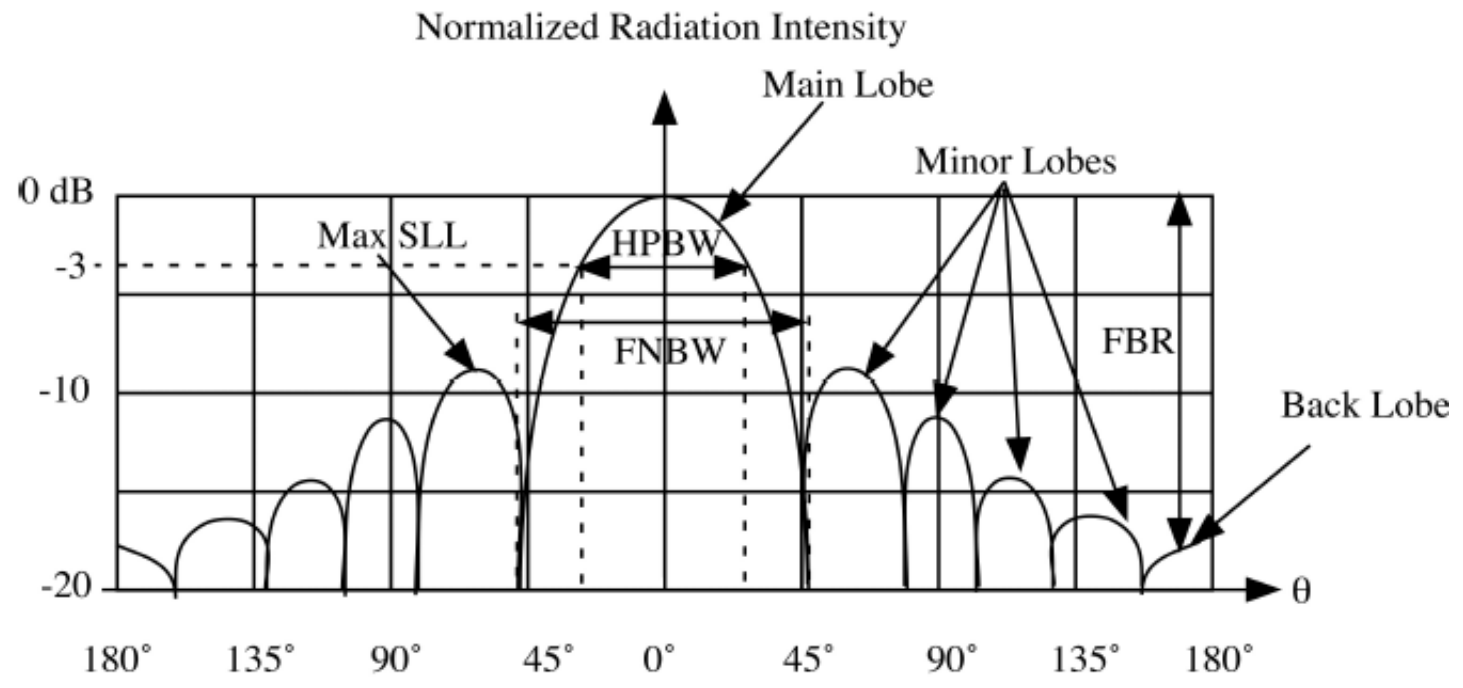

Figure 2.1: Antenna Radiation Pattern Characteristics [2].

Figure 2.1 highlights a number of important characteristics including the Half Power Beamwidth (HPBW), the Max Side Lobe Level (Max SLL), and the Front to 
Back Ratio (FBR). The normalized radiation intensity is written as:

$$
P_{n}(\theta, \phi)=\frac{P(r, \theta, \phi)}{P_{\max }}
$$

$P_{\text {max }}$ is the maximum of the radiated power and is used as a normalization factor. When $P_{n}(\theta, \phi)=0.5$ the half power beamwidth in both the $\phi=0^{\circ}$ and $\phi=90^{\circ}$ directions can be determined.

Directivity is the measure of the amount of energy in the main beam of a radiation pattern [9]. It is defined as the ratio of power in a given direction over that of averaged normalized power. The average radiation intensity is given by:

$$
P_{n, a v e}=\frac{\Omega_{p}}{4 \pi}
$$

Where $\Omega_{p}$ is the solid angle of the antennas power given by:

$$
\Omega_{p}=\iint P_{n}(\theta, \phi) d \Omega
$$

Using the normalized radiation intensity of any antenna and this value, directivity is expressed as:

$$
D(\theta, \phi)=\frac{P_{n}(\theta, \phi)}{P_{n, a v e}}
$$

With the maximum directivity expressed as:

$$
D_{\max }=\frac{4 \pi}{\Omega_{p}}
$$

For planar arrays, the directivity can be approximated using the half power beam widths in degrees of the two principal planes in the following equation: 


$$
D=\frac{32,400}{\theta_{E l} \theta_{A z}}
$$

Gain is very closely related to the directivity of the antenna, with the exception that gain takes into consideration loses. It is defined by:

$$
G(\theta, \phi)=\frac{P_{n}(\theta, \phi)}{P_{o}}
$$

$P_{o}$ is the power received into the antenna when in reception mode. There is also a relationship between aperture and the directivity of an antenna. This is defined by:

$$
D_{\max }=\frac{4 \pi A_{e}}{\lambda^{2}}
$$

$A_{e}$ is the effective aperture, therefore an electrically larger antenna will have a higher directivity and potentially a larger gain. However, there is an efficiency factor when dealing with an antenna that can be affected by non-uniform amplitude or phase and blockage among other reasons.

Grating lobes can appear in addition to the main beam, and can appear to be sidelobes. These appear when the elements in the array are spaced too far from each other, and allows for multiple directions with maximum, as given by:

$$
\Psi=k d \cos (\theta)+\beta
$$

If $d$, the distance separating elements is too large then there can be at least one grating lobe. The wavenumber is $k$, the number of waves in a certain distance. The phase progression between elements is $\beta$. Therefore if the desired effect is a low beamwidth, there is a certain point beyond which the spacing can not be widened to increase the aperture before grating lobes will appear because the spacing is too large. 


\subsection{Array Theory}

The array factor is a determining factor of the antennas radiation pattern [7]. For a planar array it is defined as:

$$
A F=S_{x m} S_{y n}
$$

Where

$$
\begin{gathered}
S_{x m}=\sum_{m=1}^{M} I_{m 1} e^{j(m-1) k d_{x} \sin \theta \cos \phi+\beta_{x}} \\
S_{x m}=\sum_{n=1}^{N} I_{1 n} e^{j(n-1) k d_{y} \sin \theta \sin \phi+\beta_{y}}
\end{gathered}
$$

Here $I_{m 1}$ and $I_{1 n}$ are the weighting of each element. The spacing is defined by the $\mathrm{dx}$ and dy variables, and the progressive phasing by beta.

$$
\begin{aligned}
& \Psi_{x}=k d_{x} \sin (\theta) \cos (\phi)+\beta_{x} \\
& \Psi_{y}=k d_{y} \sin (\theta) \sin (\phi)+\beta_{y}
\end{aligned}
$$

If the amplitude of the weightings are proportional to each direction, it can be written as $I_{m n}$, where:

$$
I_{m n}=I_{m 1} I_{1 n}
$$

This simplifies the array factor to:

$$
A F=\sum I_{m n} e_{x}^{j(m-1) \Psi_{x}} e_{y}^{j(n-1) \Psi_{y}}
$$




\subsection{Fourier Transform}

The Fourier transform can be used to approximate the near-to-far field transformation of an antenna, in particular for an array. This allows for the design of the radiation pattern in the near field. The Fourier transform can be seen below in Equation $2.22[10]$.

$$
f(x, y)=\sum_{j, k \in Z} C_{j, k} e^{i j x} e^{i k y}
$$

As seen in the array factor derivation, it is in fact a sampled Fourier transform. This connection allowed for the weighting of an array to be able to produce a square beam in a planar array.

\subsection{Duality with Digital Signal Processing Theory}

There is a duality between array design and finite impulse response filter design in DSP [1]. The duality is shown in Table 2.1: 
Table 2.1: Duality Between Signal Processing and Array Design [1].

\begin{tabular}{|c|c|}
\hline Discrete-Time Signal Processing & Discrete-Space Array Processing \\
\hline time-domain sampling $t_{n}=\mathrm{nT}$ & space-domain sampling $x_{n}=$ nd \\
\hline sampling time interval $\mathrm{T}$ & sampling space interval d \\
\hline sampling rate $1 / \mathrm{T}$ [samples/sec] & sampling rate $1 / \mathrm{d}$ [samples/meter] \\
\hline frequency $\Omega$ & wavenumber $k_{x}$ \\
\hline digital frequency $\omega=\Omega \mathrm{T}$ & digital wavenumber $\Psi=k_{x} \mathrm{~d}$ \\
\hline Nyquist interval $-\pi \leq \omega \leq \pi$ & Nyquist interval $-\pi \leq \Psi \leq \pi$ \\
\hline sampling theorem $\Omega \leq \pi / T$ & sampling theorem $k_{x} \leq \pi / d$ \\
\hline spectral images & grating lobes or fringes \\
\hline frequency response $\mathrm{A}(\omega)$ & array factor $\mathrm{A}(\Psi)$ \\
\hline $\mathrm{z}$-domain $\mathrm{z}=e^{j \omega}$ & $\mathrm{z}$-domain $\mathrm{z}=e^{j \Psi}$ \\
\hline transfer function $\mathrm{A}(\mathrm{z})$ & transfer function $\mathrm{A}(\mathrm{z})$ \\
\hline DTFT and inverse DTFT & DSFT and inverse DSFT \\
\hline pure sinusoid $e^{j \omega_{o} n}$ & narrow beam $e^{j \Psi_{o} n}$ \\
\hline windowed sinusoid $\mathrm{w}(\mathrm{n}) e^{j \omega_{o} n}$ & windowed narrow beam $\mathrm{w}(\mathrm{n}) e^{j \Psi_{o} n}$ \\
\hline frequency shifting by AM modulation & phased array scanning \\
\hline filter design by window method & array design by window method \\
\hline bandpass FIR filter design & angular sector array design \\
\hline
\end{tabular}

As identified in Section 2.5, the array factor and near field to far field transformation is a sampled Fourier transform. This relationship means that an array can be looked at in a sampled windowed point a view. It is sampled given it is discrete, and windowed because its finite. These similarities allow for an array to be designed using a similar process to that of Finite Impulse Response (FIR) filter design. 


\subsection{Squareness}

There is a number of different definitions to what a square beam is. For the purpose of this thesis, a square beam has a radiation pattern main beam that is flat in both the azimuth and elevation field cuts. It is also square when looking at it at bore sight as shown in Figure 2.2. It is important to distinquish that it is not square at every field cut, as that would produce a circular beam. With this considered the beam width is different at various field cuts.

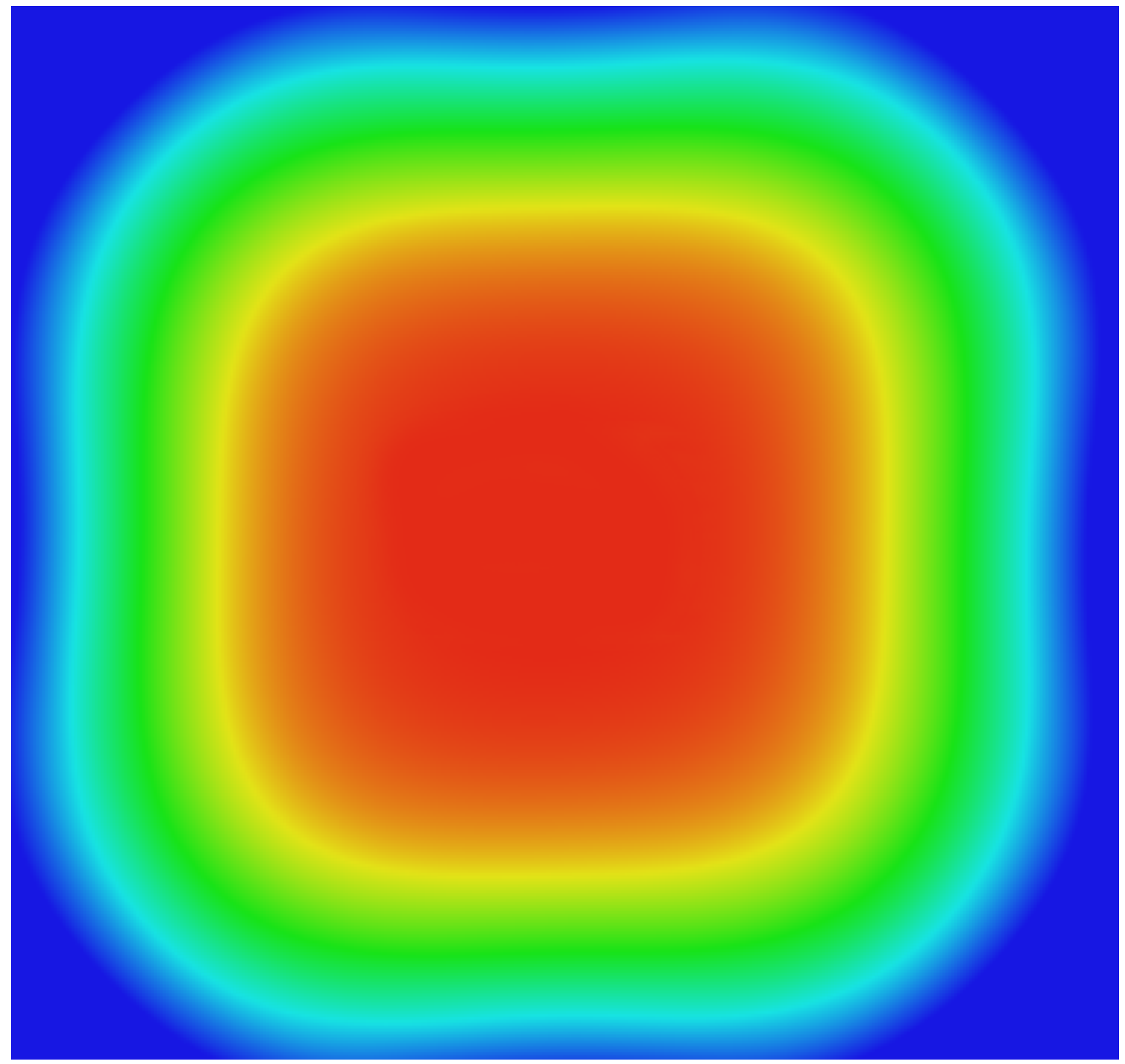

Figure 2.2: Square radiation pattern from bore sight. 


\subsection{Feed Network}

A feed network is required to take the signal from a single point and distribute it to all the patches in the array. In this thesis, a feed network is designed for an unequal, sparse array. There are three major requirements for the feed network: to provide the right power to each of the elements; to provide the right phase to all the elements; and to ensure the feed network is matched to the input in ensuring a good return loss $(\mathrm{S} 11)$.

Power dividers are used to ensure the power at each port is correct. The dividers will be discussed in 2.8.1. The lengths of microstrip lines to each antenna must be determined carefully to ensure the phases are correct. The insertion phase varies with the length of the line and at a full wavelength it cycles back to zero degrees.

Impedance matching is required to deliver maximal power to each element in the array. This can be done in a number of ways such as matching with lumped components or stubs. As long as the load impedance is real, a matching network can always be found though the performance for each different network will not be the same. Some of the factors in the selection and design of a matching network are complexity and bandwidth.

One method of matching is to use lumped components in an L- section configuration. Two configurations are possible using this method as shown below in Figure 2.3. Depending on if the normalized $\left(Z_{l}=Z_{L} / Z_{o}\right)$ load impedance appears on the inside or outside of the $1+\mathrm{jx}$ circle on the Smith chart; it is possible to determine what network to use. If it appears on the inside, the first configuration should be used, and the second if it appears on the outside.

These networks can be used with lumped components up to $1 \mathrm{GHz}$ due to the size of capacitors and inductors available [3]; however they can also be used at higher 


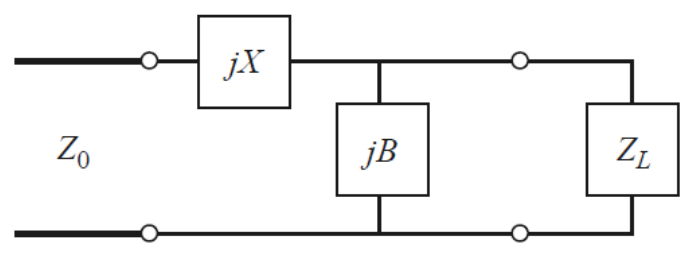

(a)

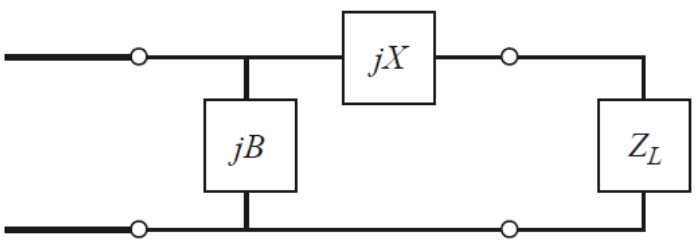

(b)

Figure 2.3: Different Layouts for Lumped Matching Techniques [3].

frequencies with the use of MMICs. These networks also have narrow bandwidth given the impedances are only correct at one frequency.

Another method of impedance matching is using stubs. These can be either opencircuited or short-circuited and can be connected in parallel or in series with the transmission line. It is also possible to have either a single stub or multiple stubs.

In single stub turning there are two parameters that can be adjusted: the distance from the load, $d$, and the length of the stub. The distance is selected so that when looking into the stub at that distance the impedance or admittance is in the form of $Y_{o}+j B$ or $Z_{o}+j X$. Then, the stubs reactance or susceptance is choosen as $j X$ or $j B$ respectively.

When dealing with microstrip or striplines, open circuited stubs are typically used because a via is not required to connect to the ground plane. A single stub network is also a relatively narrow bandwidth solution but has the advantage of not requiring any additional components.

In a corporate feed, the reflections are additive throughout the power dividers rather than having the same reflection all the way through the network. This means that multiple small reflections can become a much larger problem. Weighting of the patches also play a factor in the return loss. A lower power patch would have less of an effect on the overall return loss because of its little power compared to the overall system. A method used to combat this build up of reflections is explored in Chapter 
4.

\subsubsection{Power Dividers}

In the 1950s and 60s many different waveguide couplers and dividers were redesigned and used in microstrip technology. Following this advancement, many new types of dividers were invented and developed such as the Wilkinson power divider. [3]

The scattering matrix for a three-port network is written as:

$$
S=\left[\begin{array}{lll}
S_{11} & S_{12} & S_{13} \\
S_{21} & S_{22} & S_{23} \\
S_{31} & S_{32} & S_{33}
\end{array}\right]
$$

Some rules governing multiport junctions include that if the device is passive and is not made with any anisotropic material, then it must also be reciprocal and therefore the matrix will be symmetric. Two other characteristics are if the network is lossless and if its matched at all ports. Only two of these three characteristics can hold true at any time [3].

The simplest three port network is the T-Junction, and that can be broken down into the lossless divider and the resistive divider. In a lossless divider,the following equation needs to be true in order for it to be matched:

$$
j B+\frac{1}{Z_{1}}+\frac{1}{Z_{2}}=\frac{1}{Z_{o}}
$$

If $\mathrm{B}$ is assumed 0 , the equation reduces to

$$
\frac{1}{Z_{1}}+\frac{1}{Z_{2}}=\frac{1}{Z_{o}}
$$


In the lossless case, the characteristic impedances are all real. This type of network is shown in Figure 2.4.

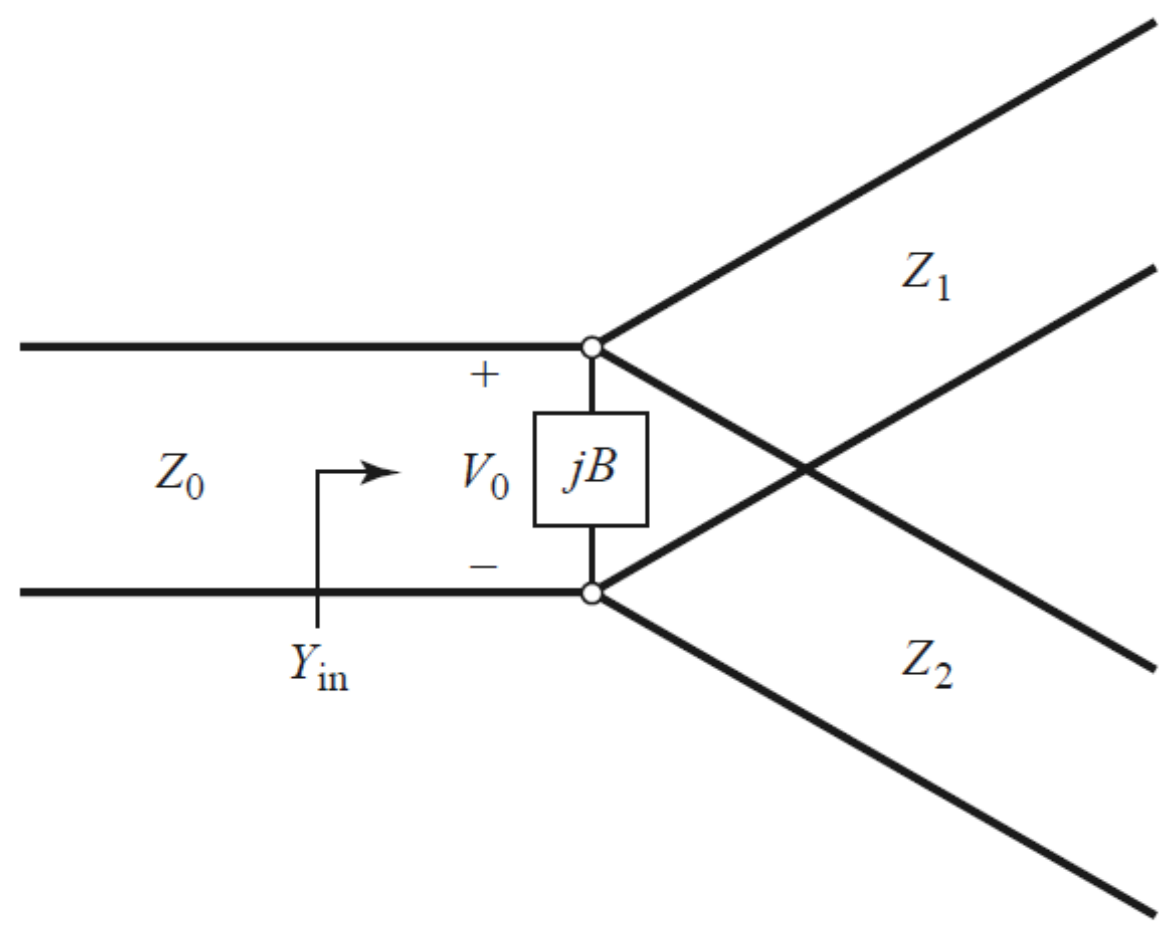

Figure 2.4: Two-way lossless power divider [3].

If a T-Junction is made with lossy components it can be matched at all ports, though there is a possibility that the output ports will not be isolated. This configuration is shown in Figure 2.5 with an equal split divider.

The scattering matrix for the resistive T-Junction divider is shown in 2.26.

$$
[S]=\frac{1}{2}\left[\begin{array}{lll}
0 & 1 & 1 \\
1 & 0 & 1 \\
1 & 1 & 0
\end{array}\right]
$$




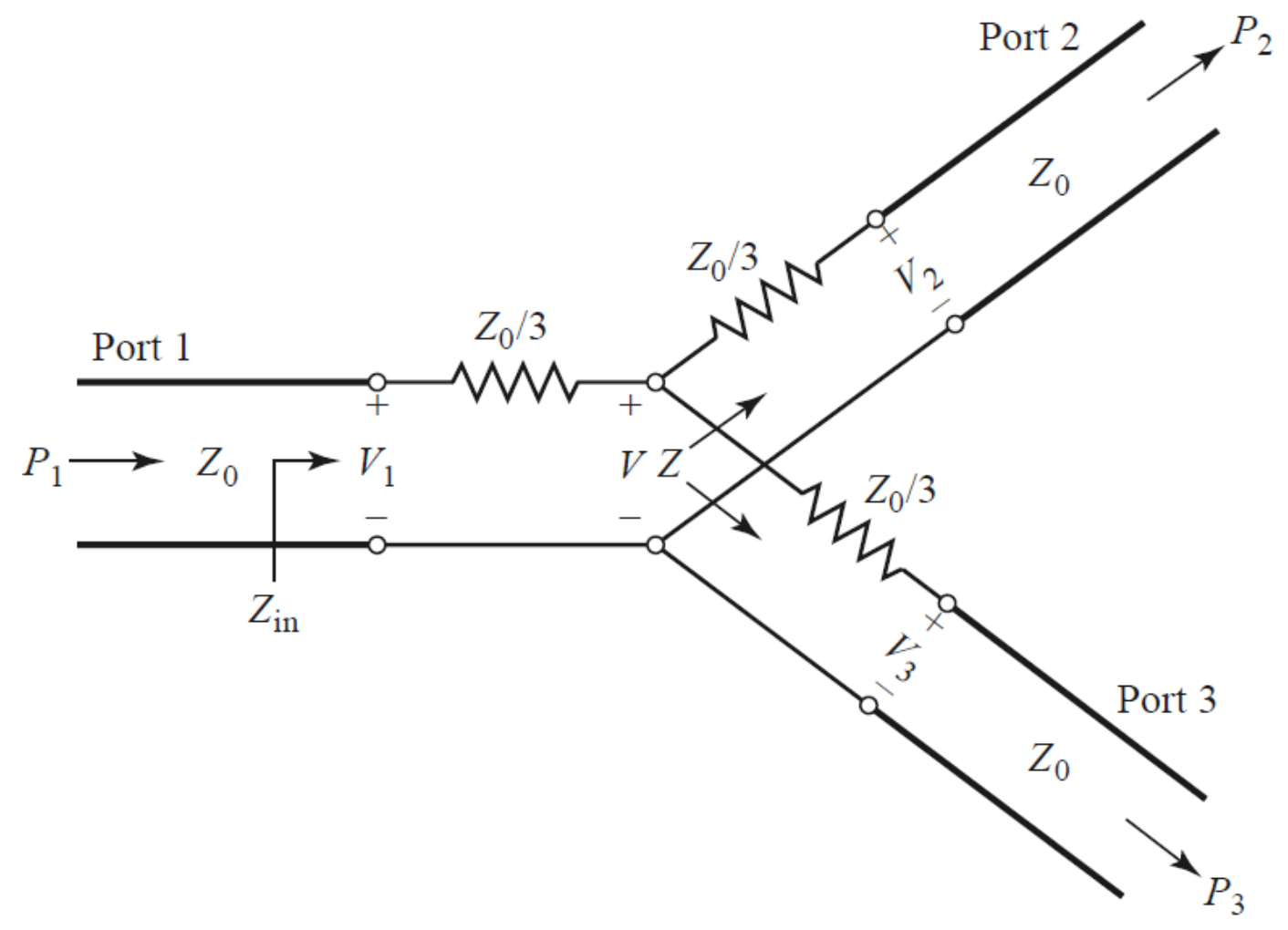

Figure 2.5: Two-way resistive power divider [3].

It is a symmetric matrix, however it is not unitary which means the system is lossy. The power at the input is written as:

$$
P_{\text {in }}=\frac{1}{2} \frac{V_{1}^{2}}{2 Z_{0}}
$$

With the output powers being written as:

$$
P_{2}=P_{3}=\frac{1}{2} \frac{\left(\frac{1}{2} V_{1}\right)^{2}}{Z_{0}}=\frac{1}{8} \frac{V_{1}^{2}}{Z_{0}}=\frac{1}{4} P_{i n}
$$

This shows that half of the power is being lost to the resistors.

A Wilkinson Power Divider is a power divider that has isolation between the output ports as well as being matched on all ports. In its simplest form, it is comprised 
of two quarter wave sections connected at one end to form the input port, and the two output ports are connected by a resistor.

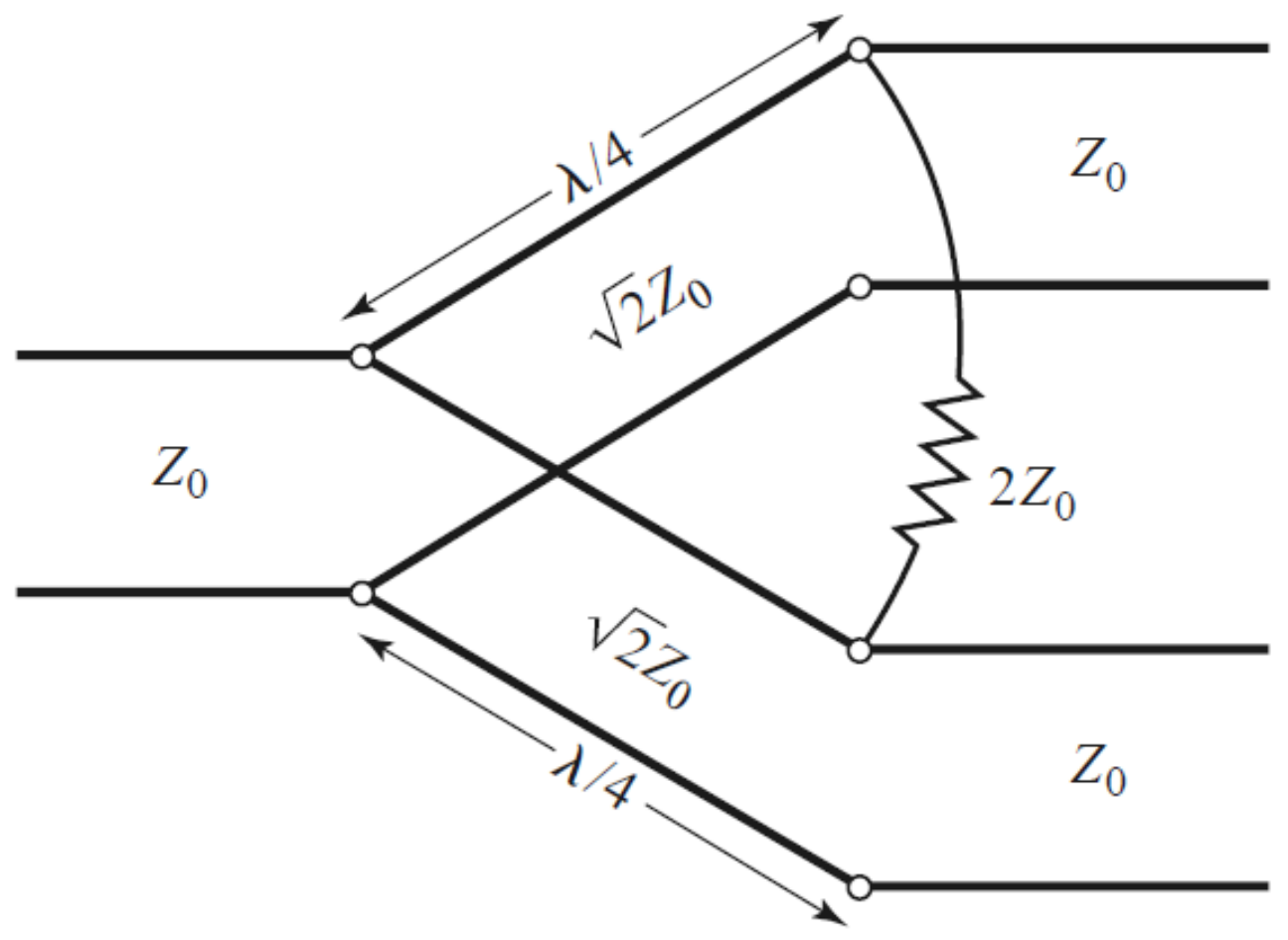

Figure 2.6: Simplified two port Wilkinson power divider [3].

In an equal divider both arms are $\sqrt{2} Z_{0}$ with the resistor being $2 Z_{0}$.

The most common way of analysis of the Wilkinson Power Divider is by even-odd mode analysis. If the impedances in Figure 2.6 are normalized to the characteristic impedance and the outputs are replaced with voltage sources, it can be redrawn as Figure 2.7. The parallel combination of the two source resistors are combined for a normalized value of 1 , this represents the impedance of a matched source. The quarter wave lines are normalized to a characteristic impedance $Z$, and the shunt resistor to a value of $r$.

The circuit is now symmetrical and can now be analyzed in even and odd modes. 


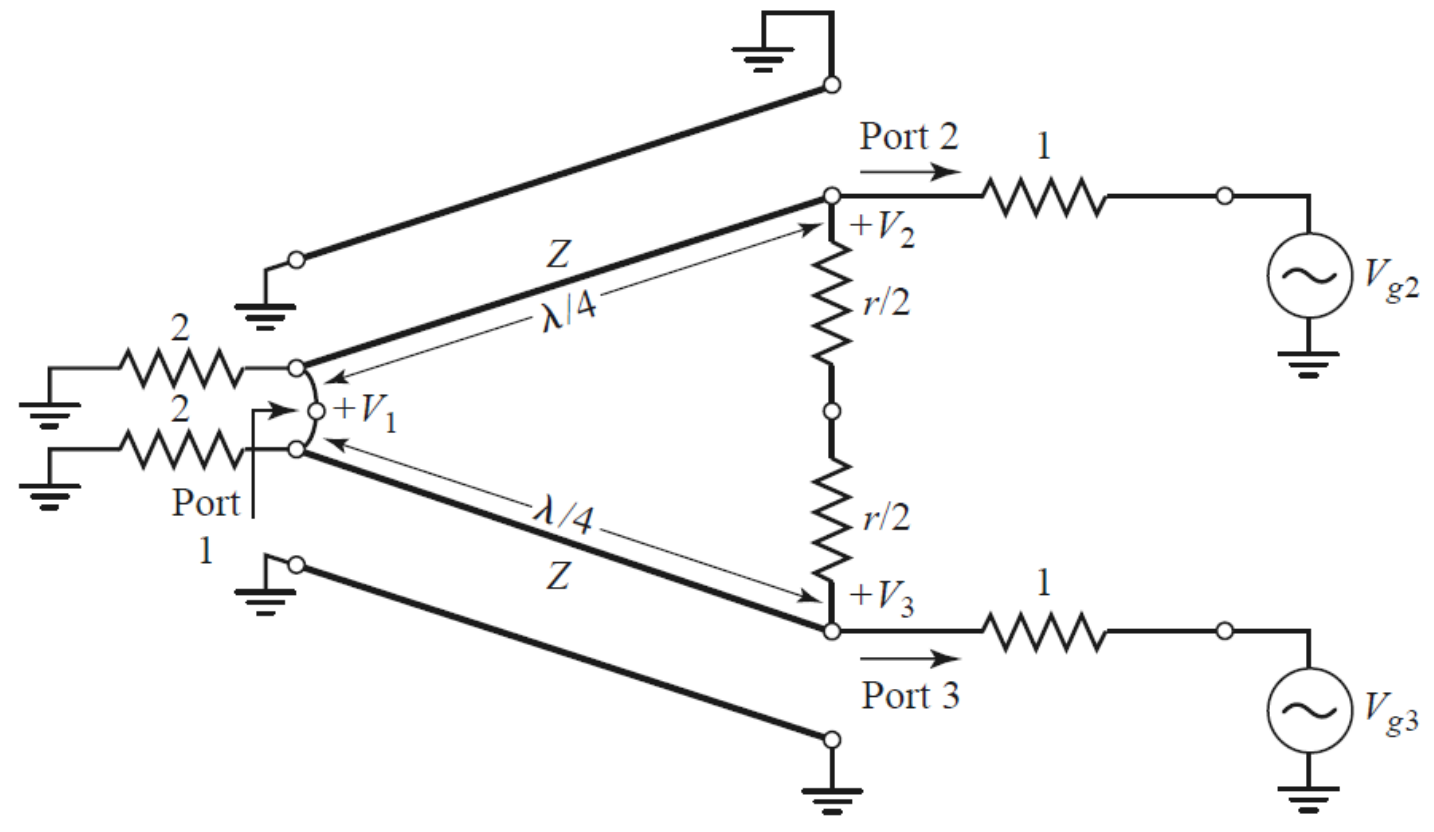

Figure 2.7: Two-way Wilkinson power divider [3].

Even mode has $V_{g 3}=V_{g 2}=2 V_{0}$, (hence the even symmetry), and odd mode has $V_{g 3}=-V_{g 2}=2 V_{0}$.

For even mode excitation, the voltage at both sources are the same. Therefore the voltage at $V_{2}$ and $V_{3}$ are equal. This means that no current flows through the $\mathrm{r} / 2$ resistors in the center of the circuit or between the short circuit at port 1 . A simplified circuit is illustrated in Figure 2.8.

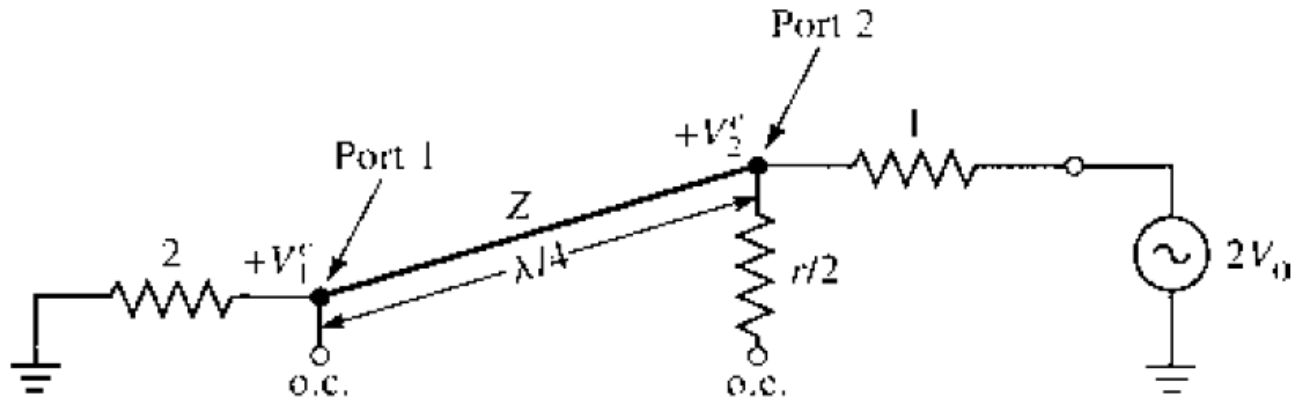

(a)

Figure 2.8: Even mode analysis of Wilkinson power divider [3]. 
Looking into port 2, an impedance of $\operatorname{Zin}=\frac{Z^{2}}{2}$ is observed because the transmission line looks like a quarter-wave transformer. If $Z=\sqrt{2}$ then $Z_{\text {in }}=Z$ and the port 2 will be matched for even mode excitation. With this $V_{2}=V_{0}$ because $Z_{\text {in }}=1$. V1 can be found using the transmission lines equations. Letting $\mathrm{x}=0$ at port 1 and a quarter wave away at port $2\left(x=\frac{-\lambda}{4}\right)$, the voltage can be written as:

$$
V(x)=V^{+}\left(e^{-j x}+\Gamma e^{j x}\right)
$$

Then

$$
\begin{gathered}
V_{2}^{e}=V\left(\frac{-\lambda}{4}\right)=j V^{+}(1-\Gamma)=V_{0}, \\
V_{1}^{e}=V(0)=V^{+}(1+\Gamma)=\frac{j V_{0}(\Gamma+1)}{\Gamma-1}
\end{gathered}
$$

The reflection coefficient is given as

$$
\Gamma=\frac{Z_{L}-Z_{s}}{Z_{L}+Z_{s}}
$$

The reflection coefficient seen at port 1 is looking at the resistor with a normalized value 2 , so it is equal $\Gamma=\frac{2-\sqrt{2}}{2+\sqrt{2}}$ and therefore $v_{1}=-j V_{0} \sqrt{2}$. For odd-mode excitation, the ports are opposite of each other with $V_{g 3}=V_{g 2}=2 V_{0}$ and $\mathrm{V} 2=-\mathrm{V} 3$. There is a null along the middle of the circuit and therefore it can be bisected by grounding it at two points along the middle plane to create the simplified circuit in Figure 2.9.

Following a similar analysis to the even mode, looking into port 2 there is an impedance of $\mathrm{r} / 2$ due to the quarter wave transmission line connected in parallel and shorted to port 1 . This looks like an open circuit at port 2, therefore will be matched for odd-mode excitation when $r=2$. All power is delivered to the $r / 2$ resistors because $v_{2}=v_{0}$ and $v_{1}=0$, none of the power then goes to port 1 . To finalize the 


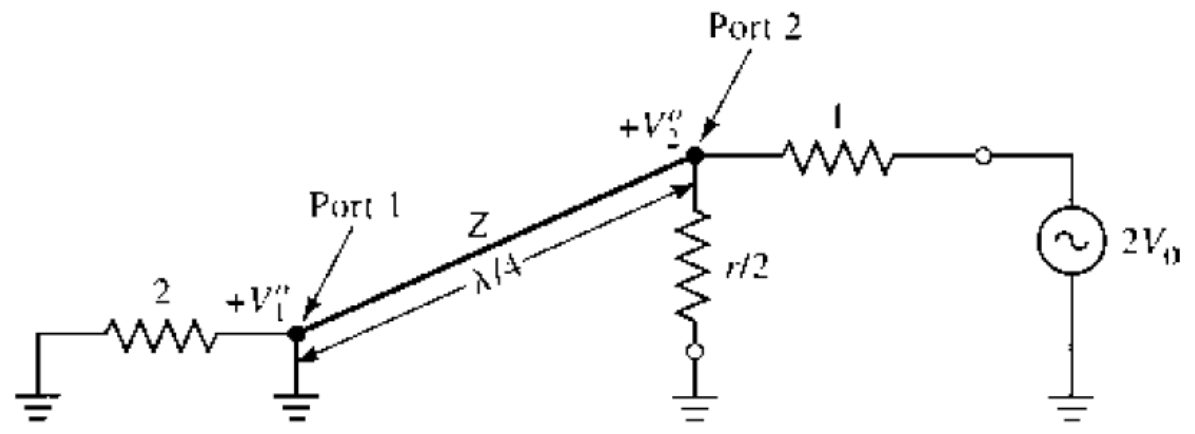

(b)

Figure 2.9: Odd mode analysis of Wilkinson power divider [3].

analysis, the input impedance at port one must be found when ports 2 and port 3 are terminated with a matched load. This is similar to the even mode excitation case because $V_{2}=V_{3}$ meaning no current flows through the resistor of normalized value 2 . The two loads are matched and therefore at a normalized value of 1 , these two loads are connected to quarter wave transformers in parallel. The input impedance is then:

$$
Z_{\text {in }}=\frac{1}{2} \sqrt{2}^{2}=1 .
$$

The scattering parameters for the Wilkinson divider are in Equation 2.34:

$$
[S]=\left[\begin{array}{ccc}
0 & -j / s q r t 2 & -j / s q r t 2 \\
-j / s q r t 2 & 0 & 0 \\
-j / s q r t 2 & 0 & 0
\end{array}\right]
$$

When the ports are perfectly matched, no power is dissipated by the resistor and therefore the divider is lossless only when the outputs are matched. Any power reflected is dissipated by the resister. Since $S_{23}=S_{32}=0$, ports 2 and 3 are isolated from each other. 
The more complex case of the Wilkinson is required when the power divided is not an equal split. Instead of having two quarter wave transformers, four are required [4]. The first two are for splitting the power unequally; hence one arm needs to have higher impedance then the other. The second set of arms transforms both back to $50 \Omega$ for the matching. The resister is in between the two sets of arms. The equations for determining the different impedances and resistances are derived below.

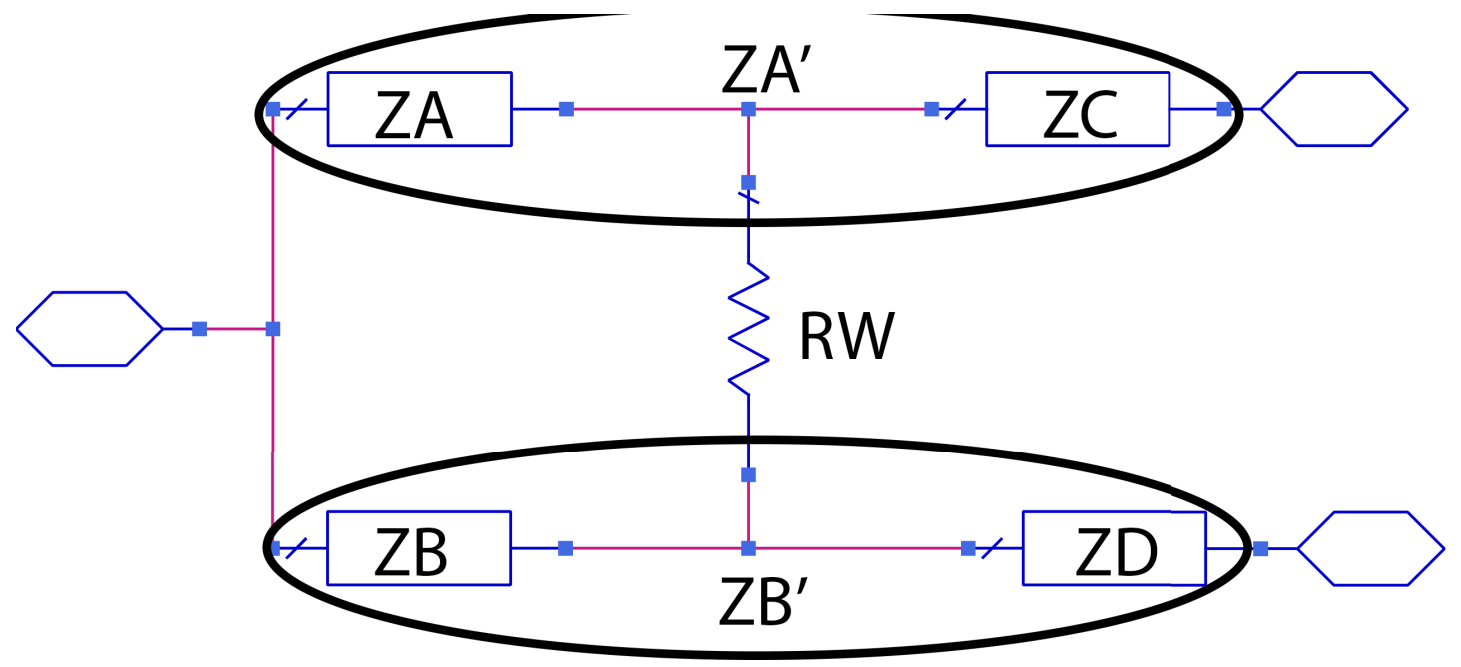

Figure 2.10: Unequal split wilkinson power divider.

The system impedance of the divider is equal to the parallel combination of each arm which can be written as ZA(ZA and ZC) and ZB(ZB and ZC) as shown in Figure 2.10. This parallel combination is written as:

$$
Z_{0}=\frac{Z A^{\prime} Z B^{\prime}}{Z A^{\prime}+Z B^{\prime}}
$$

Since the voltage at the input is the same looking into each arm, the power split is proportional to the current split as shown in Equation 2.36:

$$
\frac{P_{3}}{P_{2}}=\frac{I_{3}}{I_{2}}
$$


Where

$$
I_{3}=\frac{V}{Z B^{\prime}} \quad \text { and } \quad I_{2}=\frac{V}{Z A^{\prime}}
$$

Which simplifies to:

$$
\frac{P_{3}}{P_{2}}=\frac{Z A^{\prime}}{Z B^{\prime}}
$$

This equation can be rearranged and substituted into the equation 2.37 and solved for both ZA and ZB.

$$
\begin{aligned}
& Z A^{\prime}=Z_{0}\left(1+\frac{P_{3}}{P_{2}}\right) \\
& Z B^{\prime}=Z_{0} \frac{1+\frac{P_{3}}{P_{2}}}{\left(P_{3} / P_{2}\right)}
\end{aligned}
$$

Since both ZA and ZB are not actual component values they need to be replaced with ZA, ZB, ZC, and ZD. ZA is a two stage transformer of ZA cascaded with ZC, and $\mathrm{ZB}$ is the same with $\mathrm{ZB}$ and $\mathrm{ZD}$. This means:

$$
Z A^{\prime}=\frac{Z_{0} Z A^{2}}{Z C^{2}}
$$

And

$$
Z B^{\prime}=\frac{Z_{0} Z B^{2}}{Z D^{2}}
$$

Which when substituted into equation 2.39 and equation 2.40 gives

$$
\begin{aligned}
& Z A^{\prime}=\frac{Z_{0} Z A^{2}}{Z C^{2}}=Z_{0}\left(1+\frac{P_{3}}{P_{2}}\right) \\
& Z B^{\prime}=\frac{Z_{0} Z B^{2}}{Z D^{2}}=\frac{Z_{0}\left(1+\frac{P_{3}}{P_{2}}\right)}{P_{3} / P_{2}}
\end{aligned}
$$


In order to simplify calculations:

$$
\text { Ratio }=\frac{P_{3}}{P_{2}}
$$

Rearranging for the impedances:

$$
\begin{aligned}
& \frac{Z A}{Z C}=\sqrt{1+\text { Ratio }} \\
& \frac{Z B}{Z D}=\sqrt{\frac{1+\text { Ratio }}{\text { Ratio }}}
\end{aligned}
$$

Since the voltage across the resistor needs to be zero when power is applied from the common port, the impedance ratio must be maintained through the first two arms of the splitter: ZA and ZB.

$$
\text { Ratio }=\frac{Z A}{Z B}
$$

With equations 2.46 and 2.47 the impedance values can be solved. First ZA is set as an independent variable and the remaining impedances are solved as follows:

$$
\begin{gathered}
Z B=\frac{Z A}{\text { Ratio }} \\
Z C=\frac{Z A}{\sqrt{1+\text { Ratio }}} \\
Z D=\frac{Z C}{\sqrt{\text { Ratio }}}
\end{gathered}
$$

In order to solve for the resistor the incident power in both ports 2 and 3 must see an impedance that is matched to $Z_{o}$, as noted above in the odd mode analysis. This is shown in Figure 2.11. 


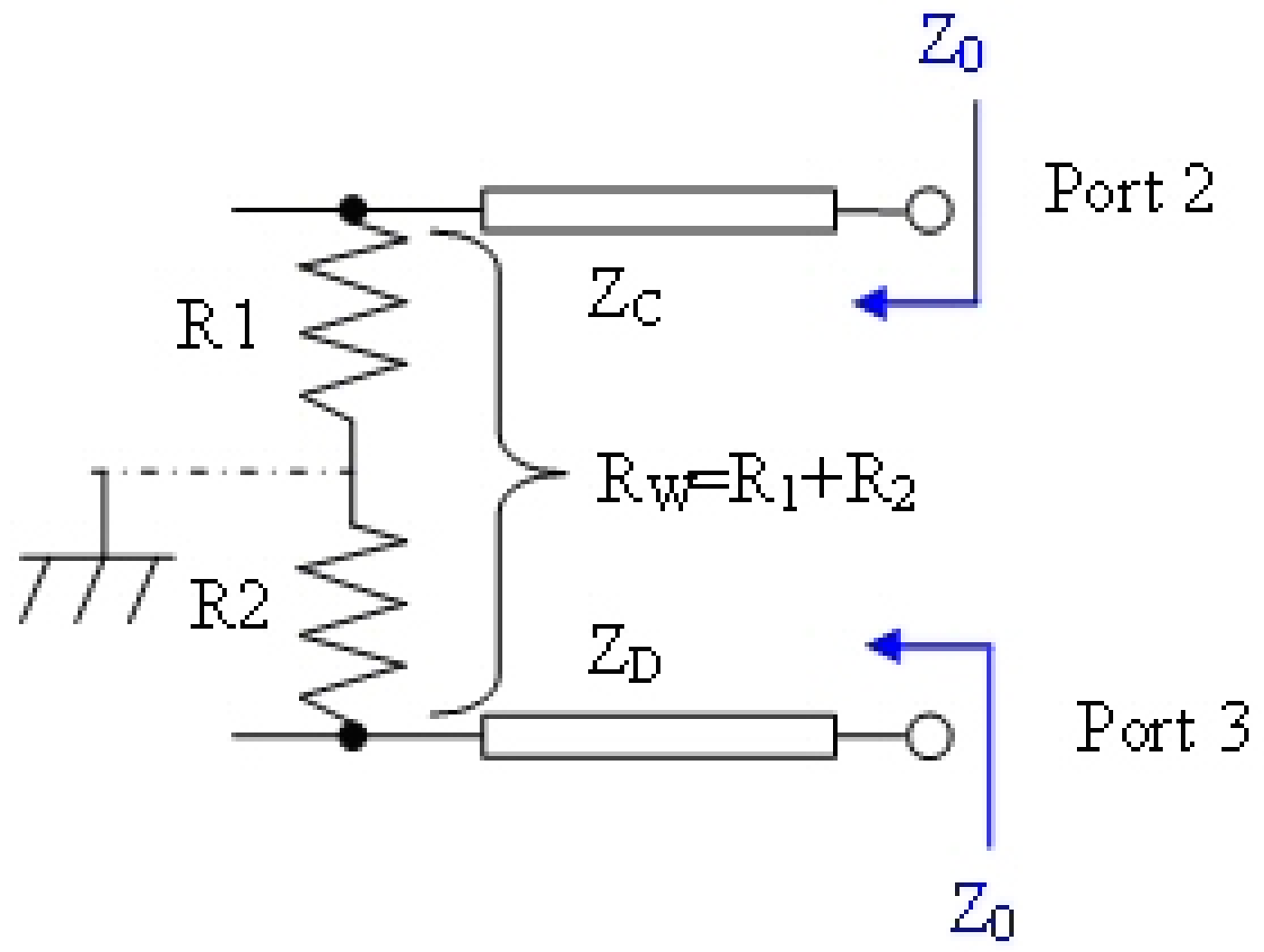

Figure 2.11: Wilkinson power divider port 2 and 3 [4].

Based on this observation:

$$
\begin{gathered}
R_{1}=\frac{Z C^{2}}{Z_{0}}=\frac{Z A^{2}}{Z_{0}(1+\text { Ratio })} \\
R_{2}=\frac{Z D^{2}}{Z_{0}}=\frac{Z A^{2}}{Z_{0}(1+\text { Ratio }) \text { Ratio }} \\
R_{W}=R_{1}+R_{2}=\frac{Z A^{2}}{Z_{0} \text { Ratio }}
\end{gathered}
$$

This method of solving for an unequal split Wilkinson power divider is different than that used in Split Tee Power Divider by Parad and Moynihan [11] in that there is a degree of freedom in the selection of one of the impedances. The major advantage 
to this method is that a designer can design the impedances to be realizable in a microstrip circuit.

\subsection{Recent Research}

This section outlines some recent work done with respect to the main components of this thesis: flat-top radiation patterns and sidelobe cancelation.

\subsubsection{Flat-Top Radiation Patterns}

Work done in the array of square or flat-top antenna radiation patterns include "Synthesis of Flat Top Power Pattern in Linear Antenna Arrays using Differential Evolution Algorithm" [12]. This research focused around a linear array for a flat-top pattern in either azimuth or elevation. The differential evolution algorithm was used to generate the weightings of the elements. In this case, a linear array is used, and likely could be expanded into a planar array. A circularly polarized array with similar properties was explored in "Wideband circularly polarised array antenna with flat-top beam pattern" [13]. This method resulted in a beamwidth of over 30 degrees. Genetic algorithm is another algorithm that can be used to generate the weightings of an antenna array as demonstrated in "Design and Realization of a Flat-Top Shaped-Beam Antenna Array" [14]. This method produced a beamwidth of over 40 degrees. Again, it was a linear array with a flat-top in only one principle plane. Further another algorithm, particle swarm optimization, is used in "Synthesis of Flat-top Beam Pattern with a Multiple Concentric Circular Ring Array Antenna" [15]. This solution created a beam that is flat in every angle and has approximately the same beamwidth (about 45 degrees), creating a circle in boresight.

This thesis covers a method for array factor synthesis using the Fourier Transform, 
for use in a linearly polarized microstrip patch sparse array as an alternative to conventional methods. The resulting beamwidth is also more narrow then the above research.

\subsubsection{Sidelobe Levels}

The recent research in flat top beams have sidelobe levels ranging from -13.2dB (manufactured [13]) to -35.1 dB (simulated [12]). Aside from optimizing weightings of antenna elements, other methods for sidelobe reduction are possible. In radar, sidelobe cancellation is often done in order to create a null in a location with a lot of interference [16]. This method has an auxiliary antenna that has its radiation pattern line up with that of the sidelobe and through DSP actively cancels out the interference on that sidelobe.

This thesis explores a reactive yet passive method for sidelobe reduction in a sparse array, without changing the weightings of the initial array elements. 


\section{Chapter 3}

\section{Array Factor Design}

\subsection{Introduction}

The design of a square beam antenna with low sidelobes allowed for many different variables in the design. From the beginning, Ericsson laid out the initial targets for specifications. These are:

1. $2400-2483 \mathrm{MHz}$ bandwidth

2. Ideal Beam width of 20x20 degrees

3. $600 \mathrm{~mm} \times 600 \mathrm{~mm}(24 " \mathrm{x} 24 ")$

4. Gain $>18 \mathrm{dBi}$

5. First Sidelobe Suppression $>30 \mathrm{~dB}$

6. Return Loss $>12 \mathrm{~dB}$

7. Patch Design

8. FR4 Patch Design

9. Rogers RO-4003 Feed Network 
10. Square Beam Radiation Pattern (in Bore sight)

Variables were minimized given the material and antenna type were selected. The patches were previously designed by Petra Microwave to allow for cross polarized Multiple Input Multiple Output (MIMO). The patches were fed directly with the desired amplitude and phase. This section focuses on the array factor.

\subsection{Simulation Tools}

The primary tool used in simulation is Empire XPU. As a time domain simulator, it is effective in simulating a range of frequencies instead of a single one as in frequency domain simulators. It is used to do full wave 3D simulations. Setup instruction are found in Appendix A.

Mathworks MATLAB was used to do the initial simulation to determine the size of the array. In particular, the phased array toolkit was used to quickly simulate the array factor. The code used to do this is in Appendix B. MATLAB is used in Section 3.3 .

Agilent Advanced Design Systems (ADS) was used in the initial feed network simulations. It was also used for the design and verification of the power dividers. As described in Section 4.3, ADS was selected given its ability to tune and view simulation results on the fly rather than performing a full wave 3D simulation which is time consuming.

\subsection{Square Patch Array}

In order to test the model that a sinc function in the near field would produce a square beam in the far field, a number of equally spaced square arrays were simulated using 
MATLABs phased array tool kit. After running a number of simulations with different sized arrays, it was determined that a 9x9 array with a sinc function weighting could produce a square beam in both the azimuth and the elevation; however, the sidelobe levels were too high. These trials can be seen in figure 3.1 with arrays up to a $13 \times 13$ array. With this tool, the spacing of elements can easily be manipulated: a spacing of $0.7 \lambda$ produced the ideal shape and minimized sidelobes.

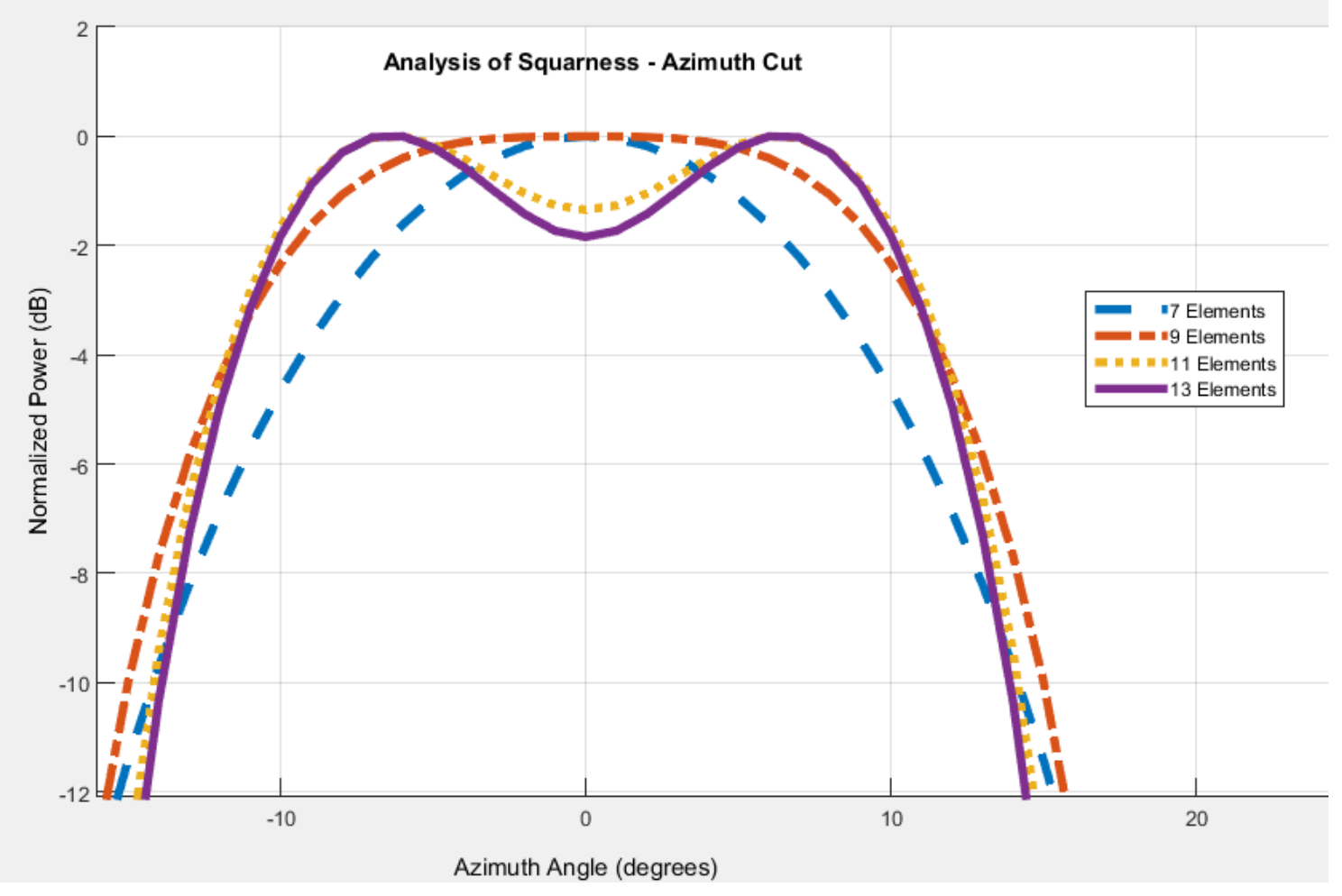

Figure 3.1: Level of squareness for 4 different sized arrays. 


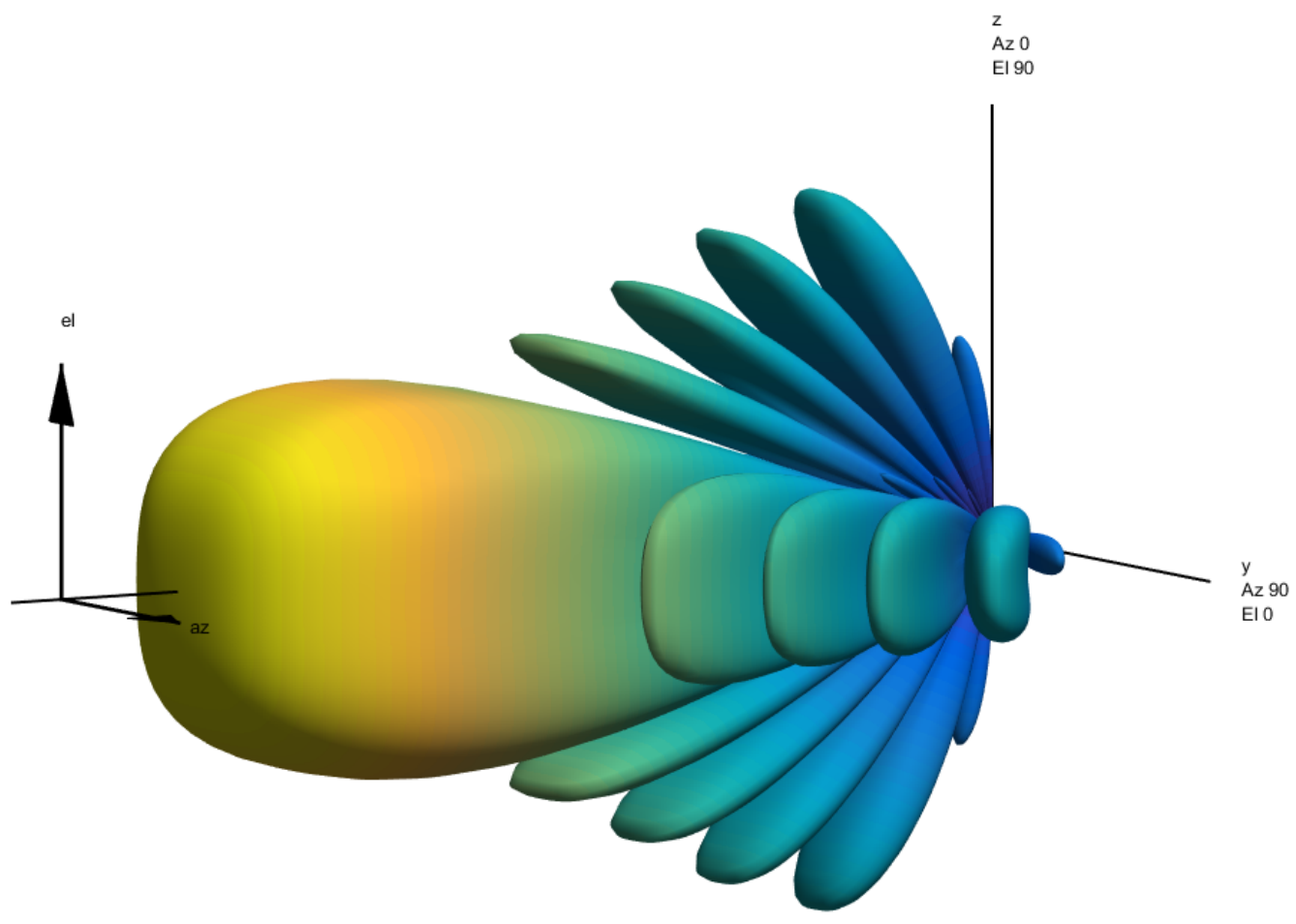

Figure 3.2: 3D Plot of 9x9 Array.

Figure 3.1 shows that a 9x9 array produces a very flat mainlobe and a square in boresight as shown in Figure 3.2. A 7x7 array was not sampled enough to incorporate the first sidelobe of the sinc function, this led to a square beam not being created. Both 11x11 and 13x13 produced a beam with a ripple in the middle. The results for the sidelobe performance is illustrated in Figure 3.3. 


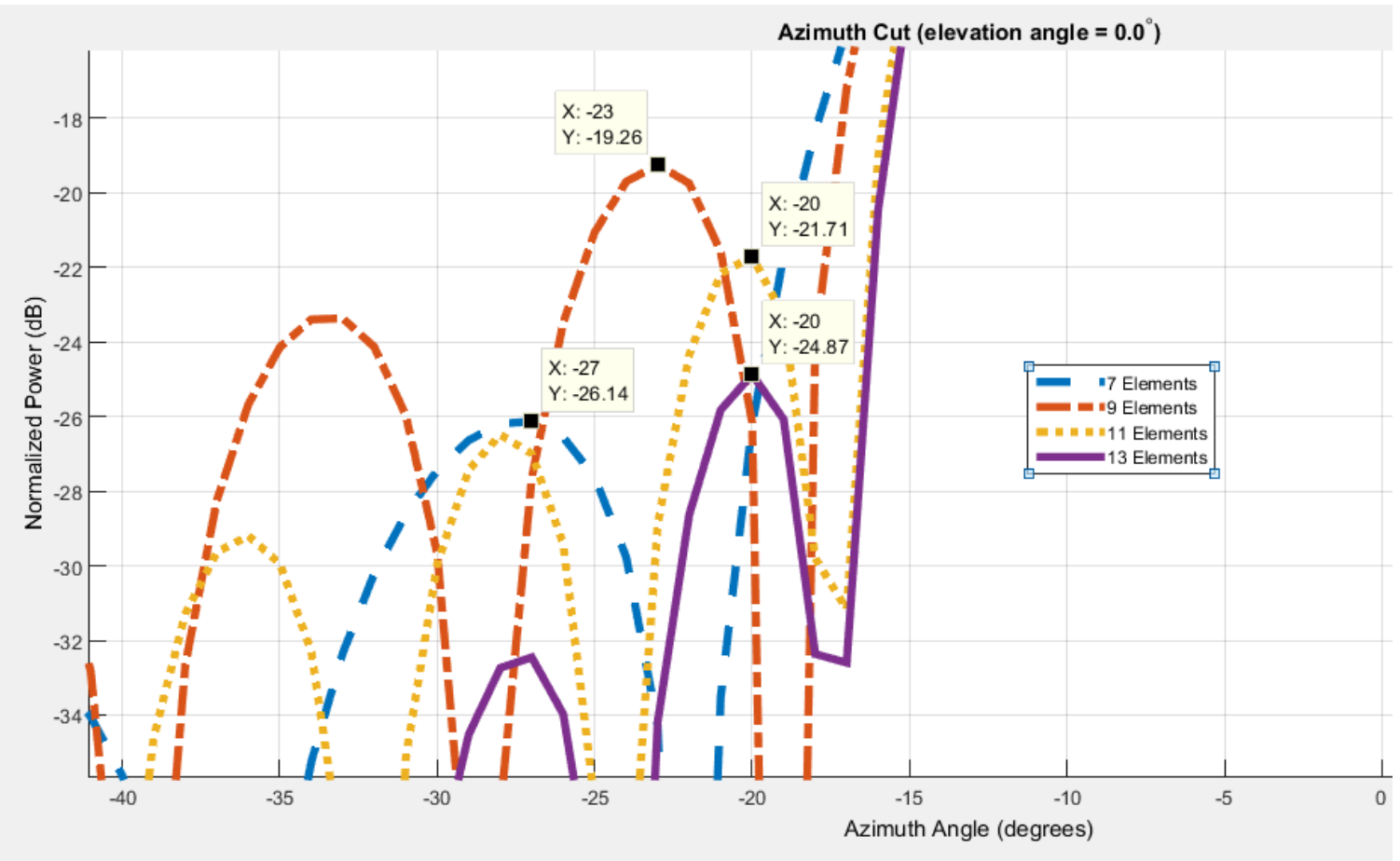

Figure 3.3: Sidelobe level for 4 different sized arrays.

The results show that the $7 \times 7$ array had the lowest sidelobes but not the square beam desired. The ideal square beam of the 9x9 array also had the highest Max SLL. It was observed that with more elements in the array, the sidelobes became lower.

The weighting of all the arrays were identical with the exception that adding more elements increased the window of the sinc function being sampled. The 9x9 array was selected because it was the smallest size that produced a square beam. The weightings used in this configuration are shown in Figure 3.4. These weightings are rounded and are in current when imputed into Empire. 


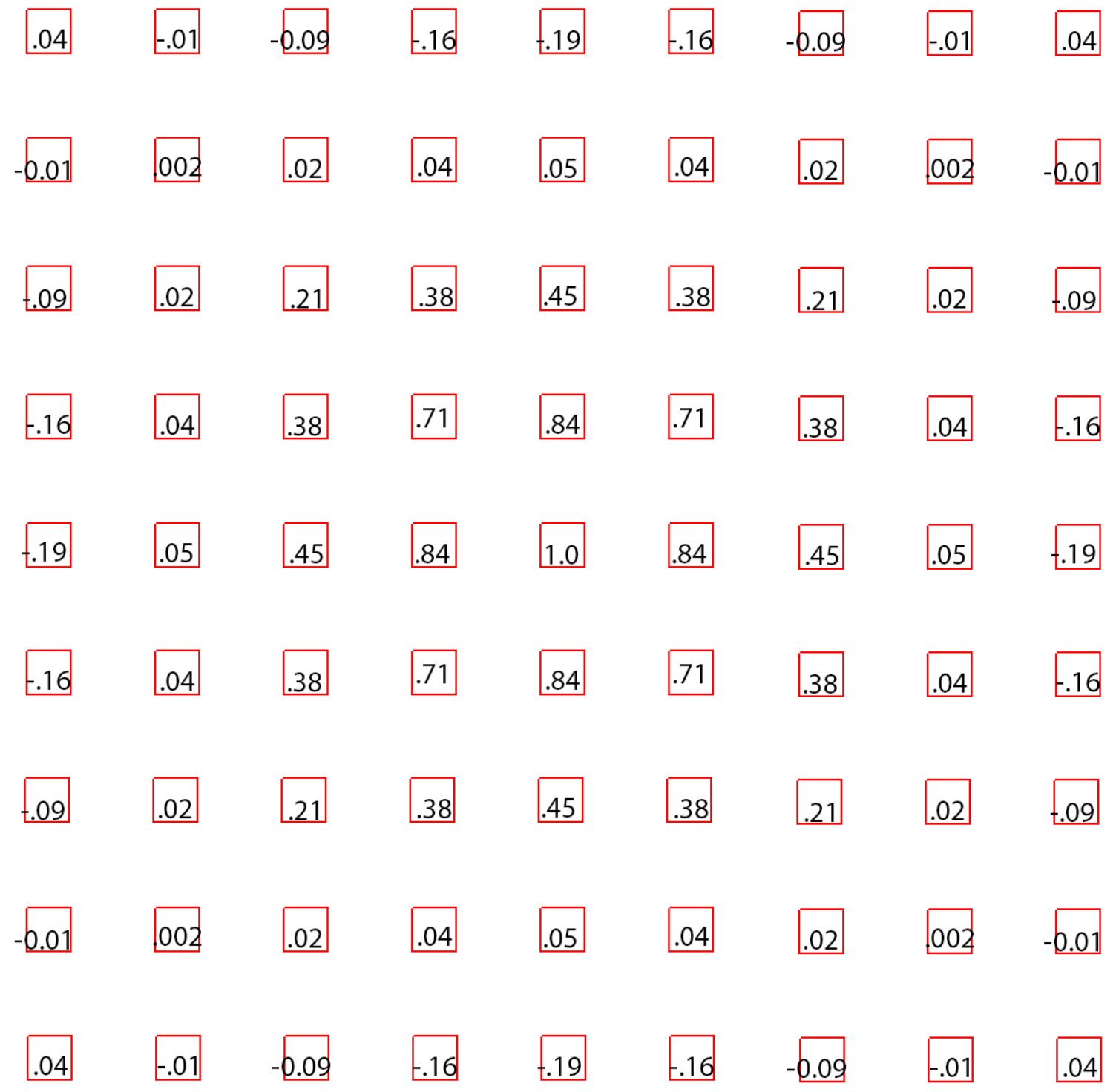

Figure 3.4: Layout of $9 x 9$ array with weighting.

An electromagnetic simulation was completed using Empire to verify the results in Figure 3.5. The patches were directly excited by ports with specific weighting. The radiation patterns for the remainder of the thesis are a combination of the co-polarized and cross-polarized patterns or EABS. 


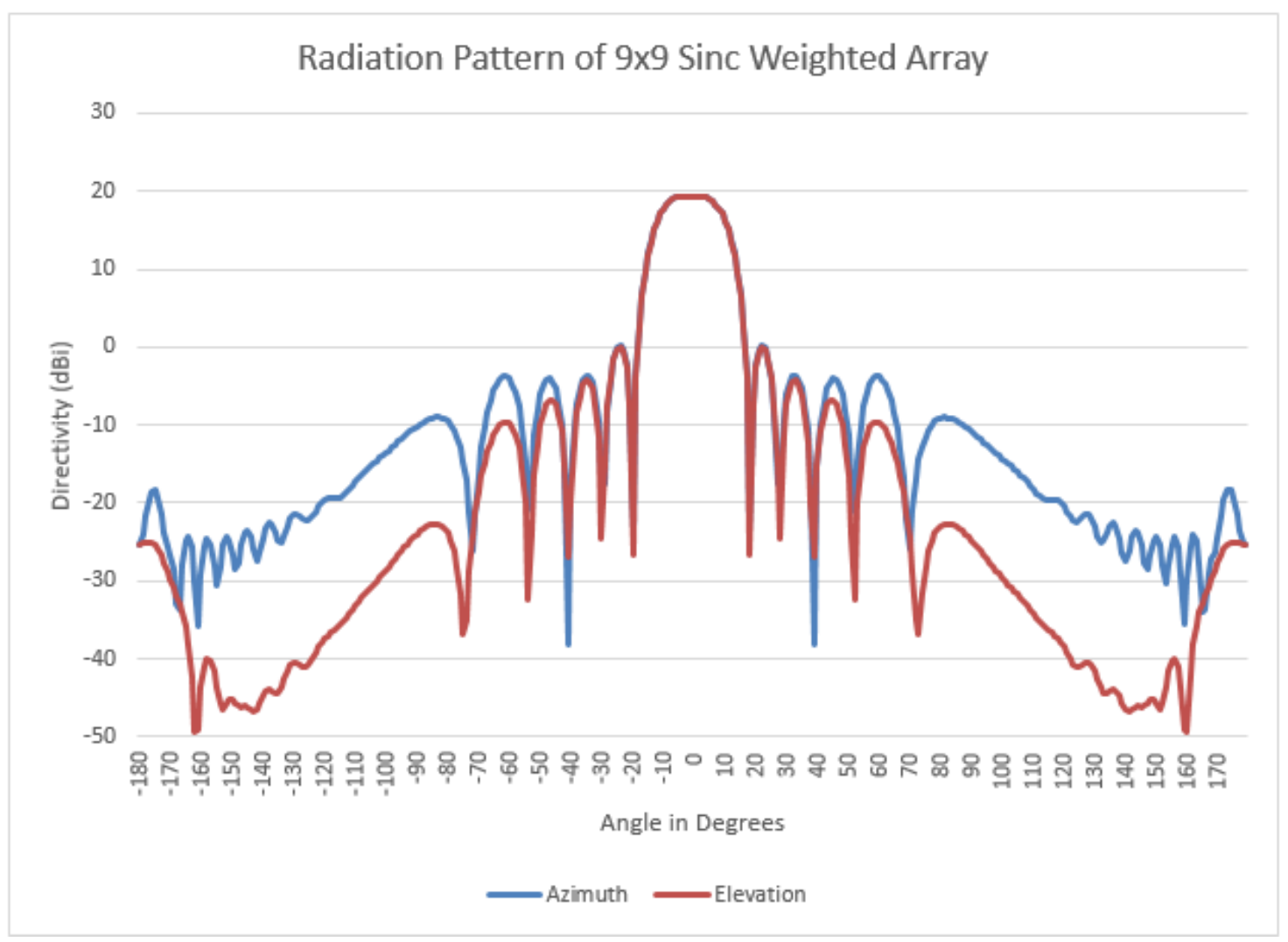

Figure 3.5: Radiation pattern of $9 \times 9$ array with sinc weighting.

In this case, the sidelobes were still too high but the main lobe remained a square as in the MATLAB simulations. The azimuth and elevation radiation pattern cuts are different because the patches are being fed on the side. This simulation confirmed the MATLAB array factor results. The beamwidth was too large due to the restrictions based on the aperature size. The feed network would also be complex due to the unequal power and number of elements required. Reduction in sidelobe levels and complexity were the primary concerns and drove the rest of the array design stage. 


\subsection{Array Reduction}

In order to reduce the overall number of elements, any element that received less than $5 \%$ of the power delivered to the center element was removed with the exception of the negative weighted patches. This resulted in the layout in Figure 3.6.

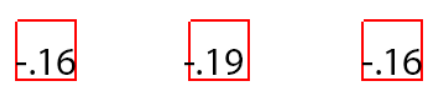

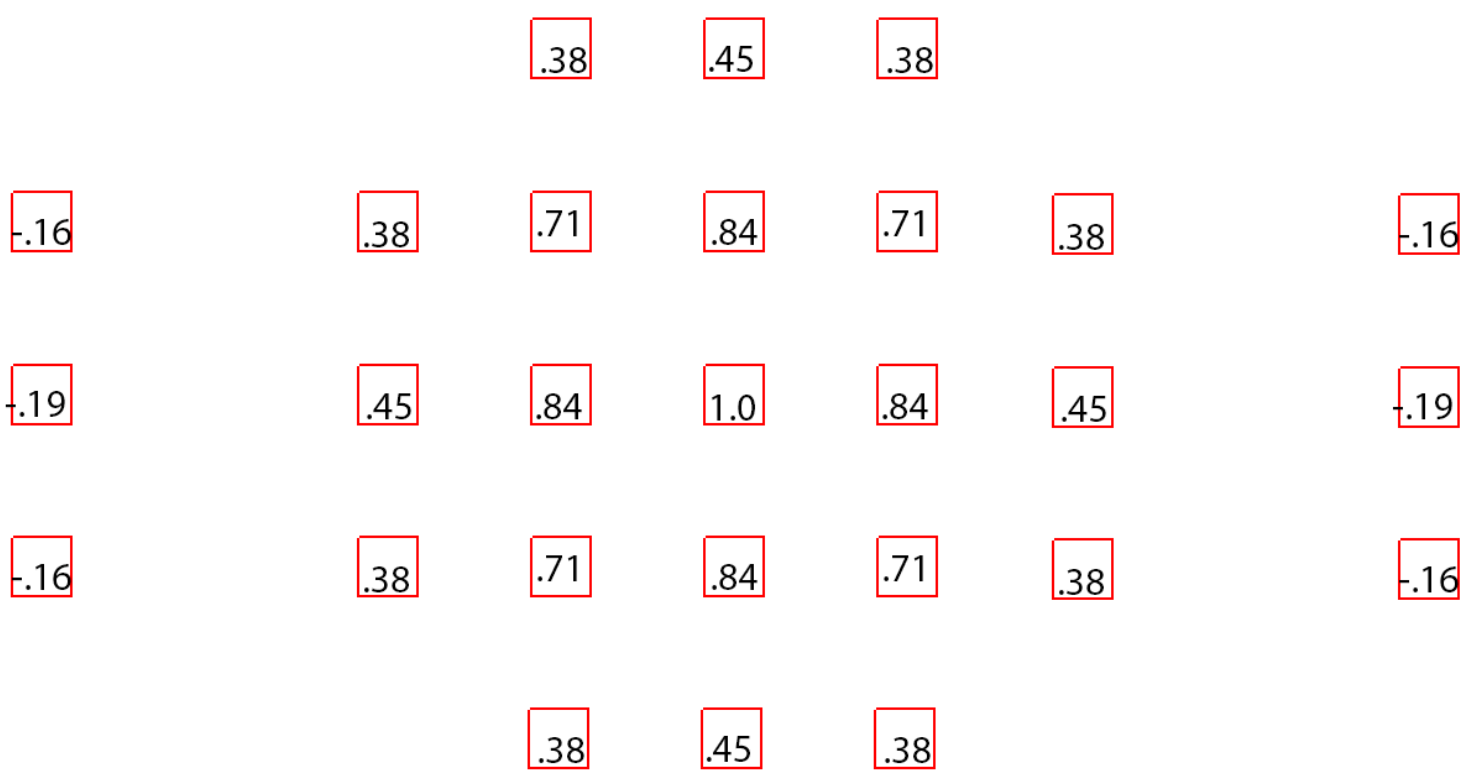

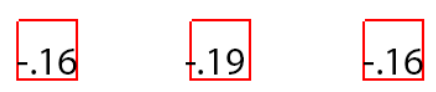

Figure 3.6: Layout after removing low power elements.

This reduced the array from 81 element to 33 elements-a reduction of $59.2 \%$ and produced results that were nearly identical to those found in the full $9 \mathrm{x} 9$ array as 
shown in Figure 3.7.

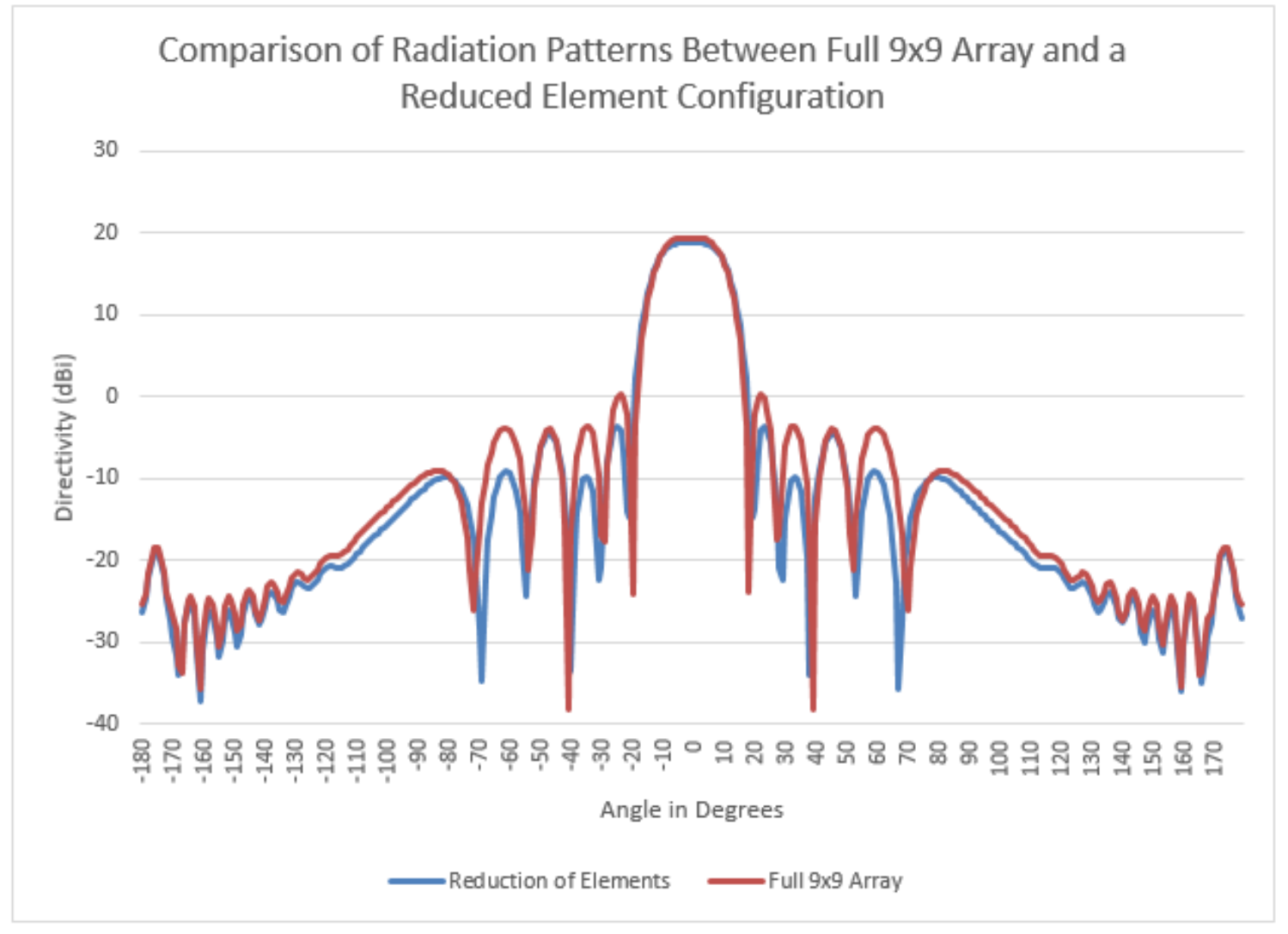

Figure 3.7: Radiation pattern after removing low power elements.

While this approach lead to a reduction of total elements, it was still too large and caused an overly complex feed network due to the unequal power splitting. A simpler feed network could be created by using only equal power splits; therefore, the next step was to move to equal weighting of elements. If the combined power of two elements was $1 \mathrm{~A}$, then the combination of the two would be fairly similar when replaced with a single patch with a weighting of $1 \mathrm{~A}$. In general, this method does not work because it fails to account for spatial distribution. It was still explored as a method to reduce the number of elements. Working from the centre outward, this process was used in a few iterations with results in Figure 3.8. 
\begin{tabular}{ll}
-.16 & $.19 \quad .16$ \\
\hline
\end{tabular}

\begin{tabular}{lll}
.38 & .45 & .38 \\
\hline
\end{tabular}
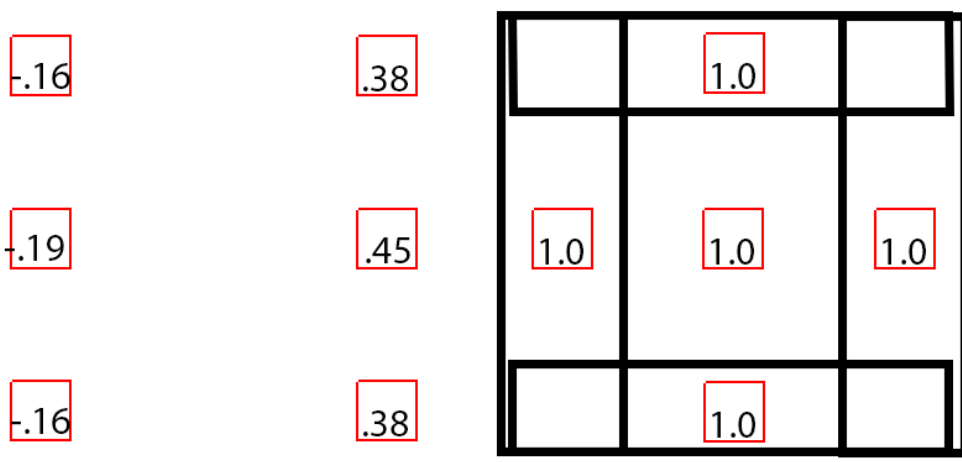

.38

$-19$

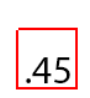

$-.16$

.38

\begin{tabular}{l}
.38 \\
.45 \\
.38 \\
\hline
\end{tabular}

.38

.45

.38

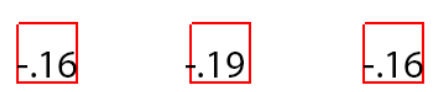

Figure 3.8: Layout with uniform weighting in center.

Here, the four merged patches are set to a weighting of $1 \mathrm{~A}$ and a phase of 0 degrees, with the remaining patches still set to the values of the sinc function. The next reduction merged the remaining groups of patches with a weighting of $1 \mathrm{~A}$, and the "squaring" outlier patches merged to a weighting of $-0.6 \mathrm{~A}$, resulting in the layout in Figure 3.9. A negative weighting can be realized by applying a phase shift of 180 degrees at the port. 


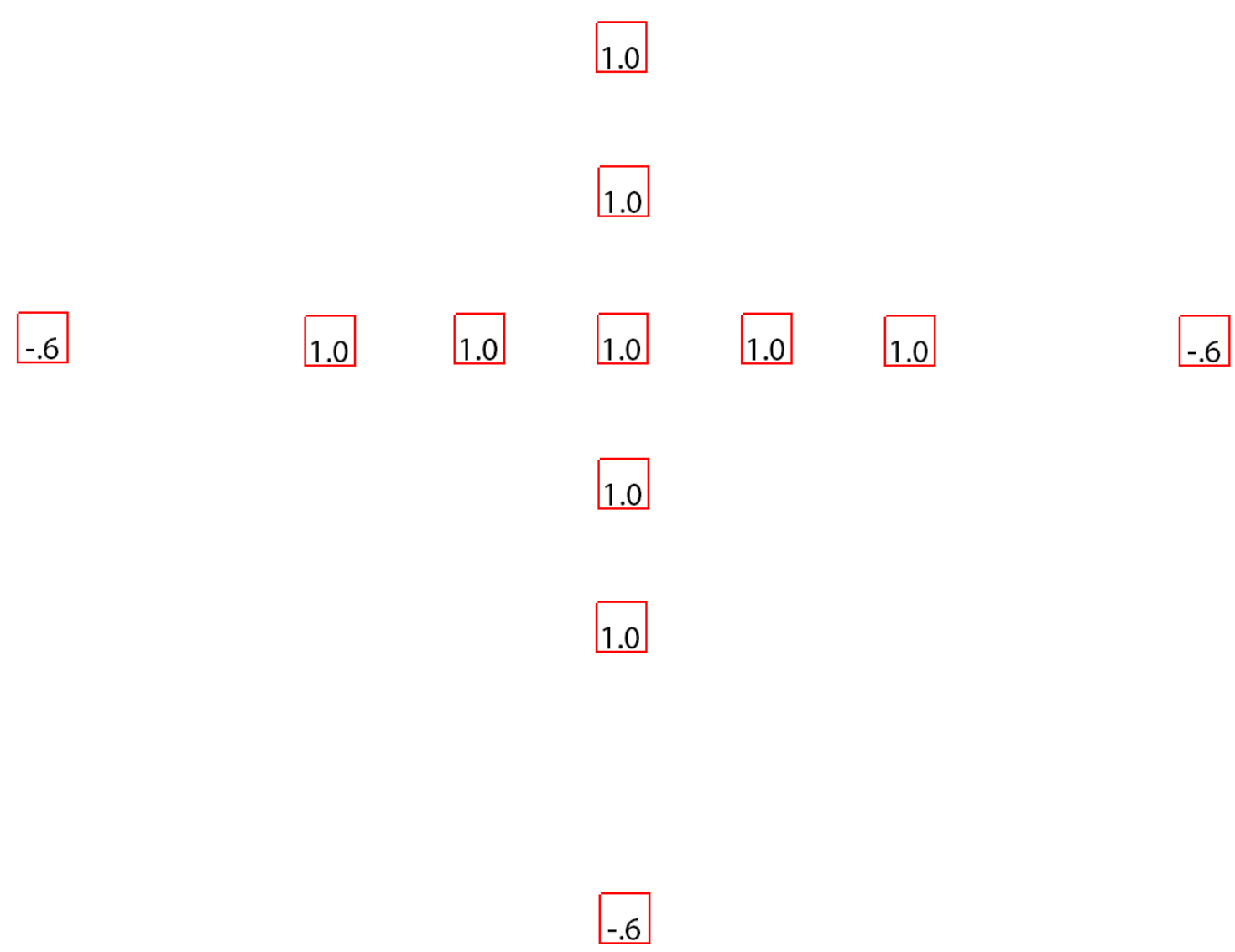

Figure 3.9: Layout with uniform weighting in cross elements.

The cross shape had the lowest number of patches of all the different tests with only 13 elements, a reduction of $84 \%$ from the full $9 \times 9$ array. It also had the advantage of being uniformly fed with the exception of the outside four patches. The downside was the sidelobe levels were too high as indicated in Figure 3.10. 


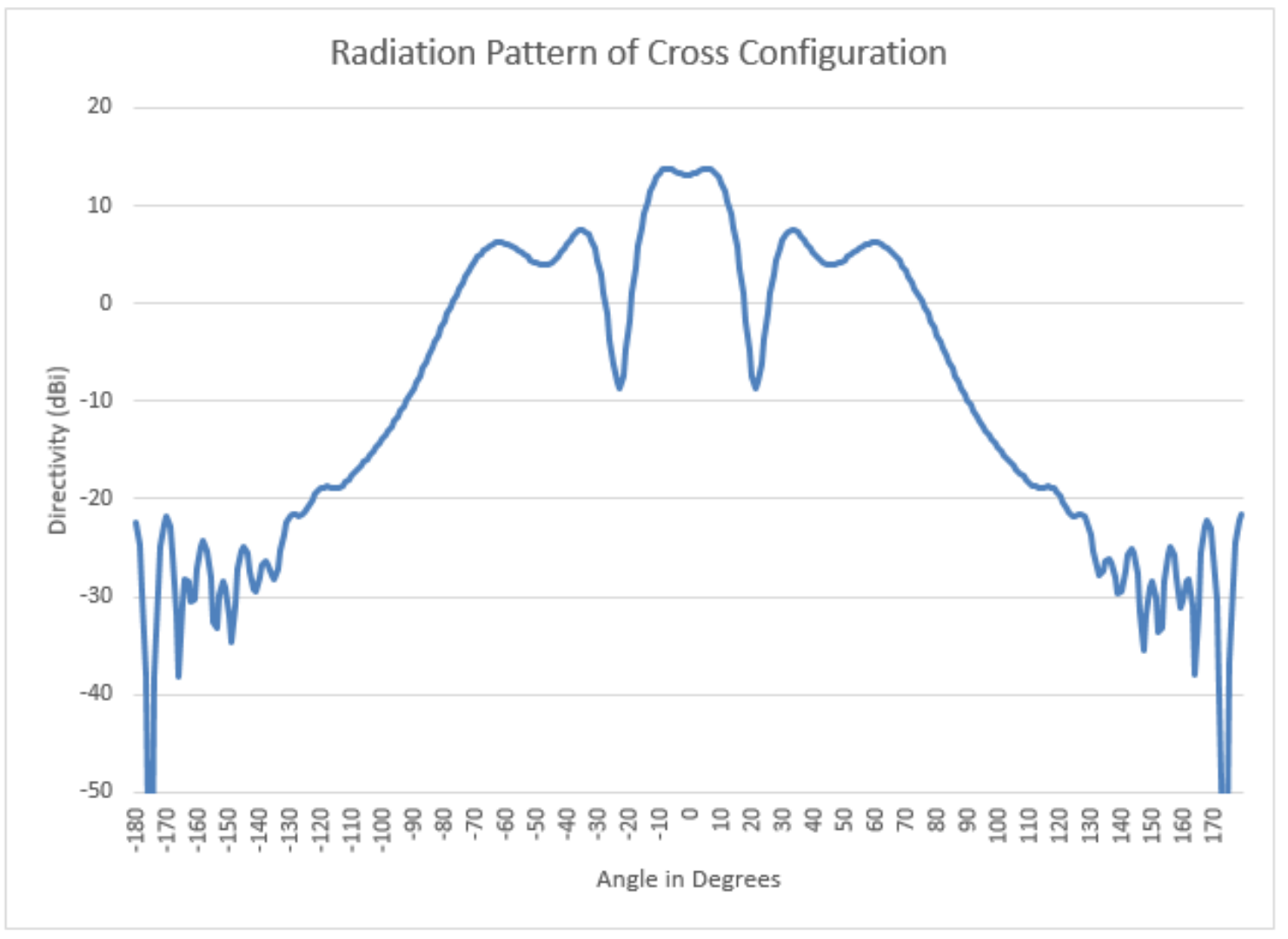

Figure 3.10: Radiation Pattern with uniform weighting in cross elements.

The far-field to near-field transformation is the same function as a sampled Fourier transform. This means that when the field in the near-field resembles a sinc function, it will be a square in the far field. When the sinc function is overlaid on top of the layout it is clear that there are not enough sample points to produce ideal square beam as shown in Figure 3.11. 

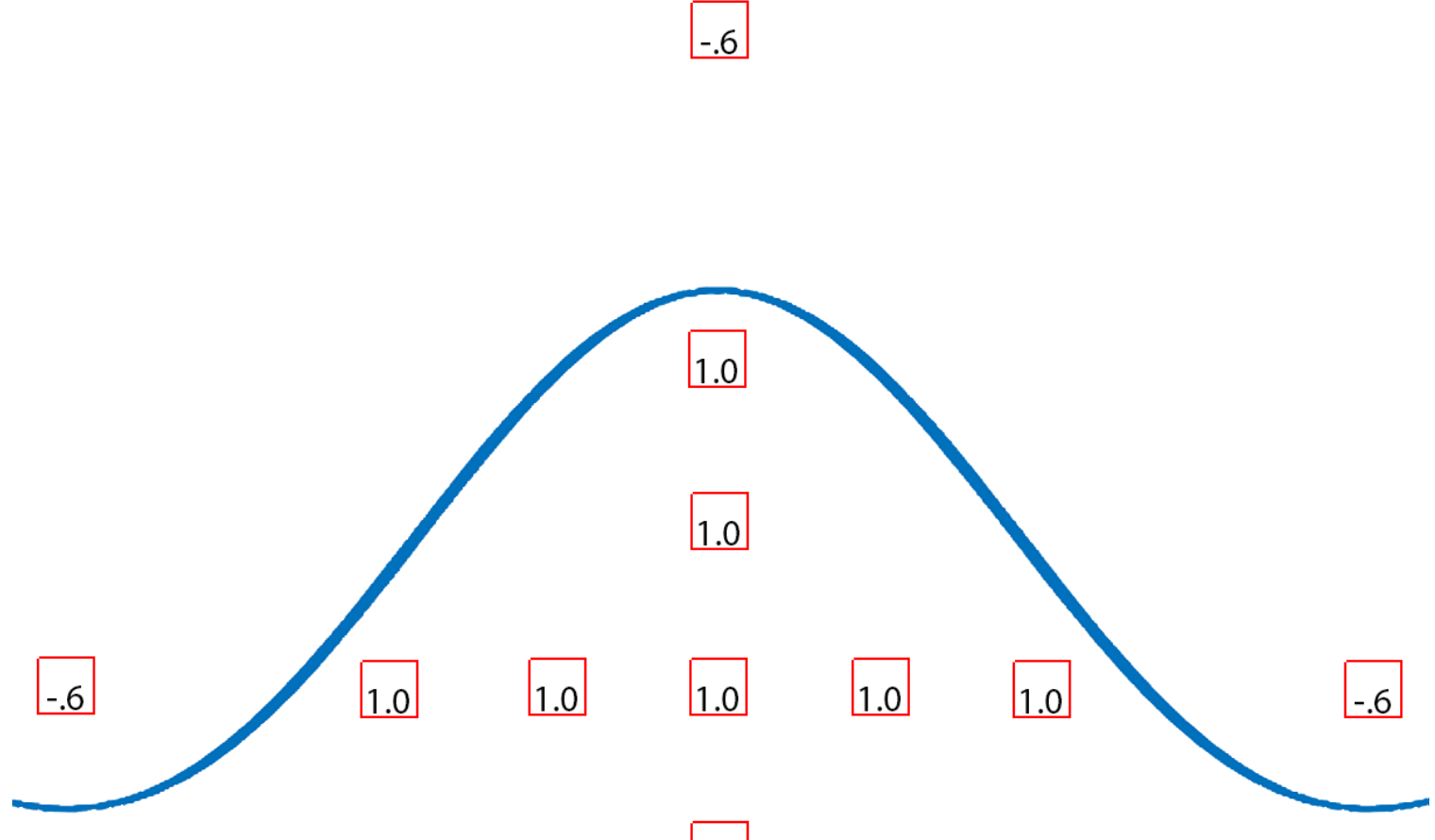

1.0

1.0

$-.6$

Figure 3.11: Sinc function overlaid over layout of cross.

If patches are added on a diagonal from the center, then the inherent resolution of the sinc function would be finer and therefore the transform more ideal. This layout can be seen in Figure 3.12. 


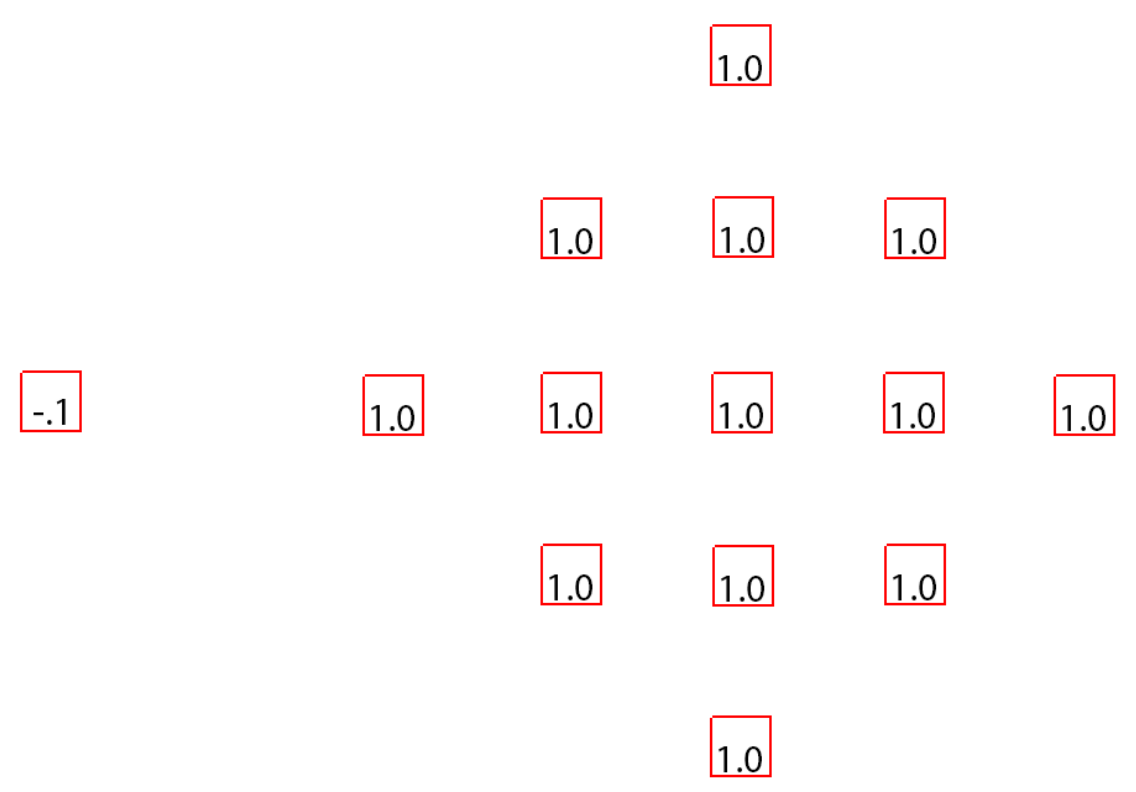

Figure 3.12: Layout with uniform weighting in a sinc pattern.

This resembles a sampled sinc function more closely and produced similar results to the $9 \times 9$ element array with a fraction of the elements as shown in Figure 3.13. 


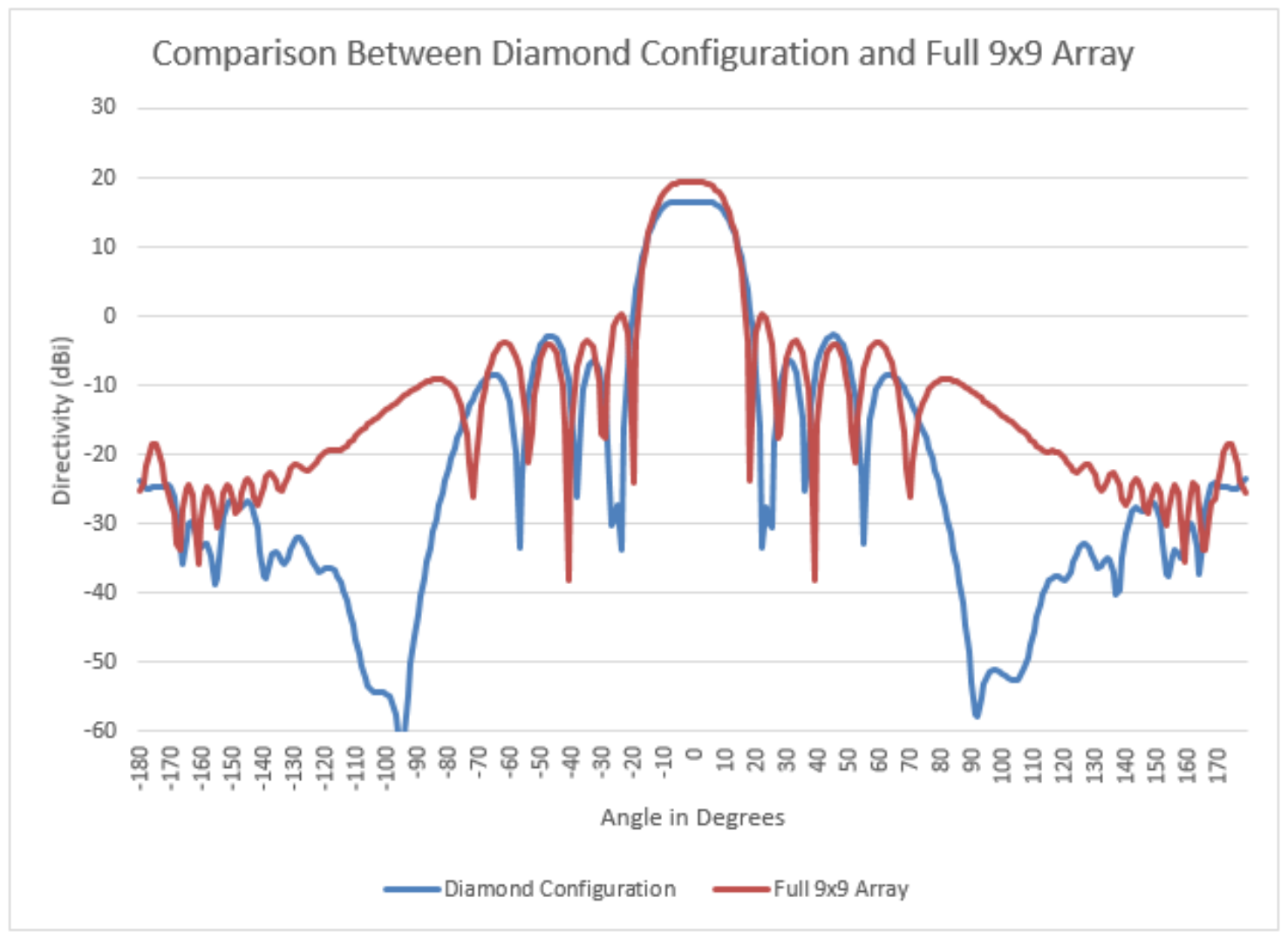

Figure 3.13: Radiation Pattern with uniform weighting in a sinc layout.

The major difference between the plots above is the directivity of the full array is greater than that of the reduced element; however, the sidelobe levels were approximately the same just at different angles. The placement of the elements resemble the shape of a sinc function. The near field radiation would be similar to that of the full array with a fraction of the elements as well as equal power weighting. The inverted power of the left most patch can be seen as the first sidelobe of a sinc function. The power concentration increases from left to right in the array in the same manner as the power in a sinc function as shown in Figure 3.11. Since the array is symmetrical, it remains true in both the Azimuth and Elevation planes. The results are very similar to that of the 9x9 array but with far fewer elements. It also allowed for the 
reduction in overall size due to its diamond shape. This patch layout was used for the remainder of the research, since it provided the correct size and minimized antenna elements.

\subsection{Gaussian Weighted Sampled Sinc Function}

To reduce the sidelobes even further, a Gaussian weighting was applied as shown in Figure 3.14. 


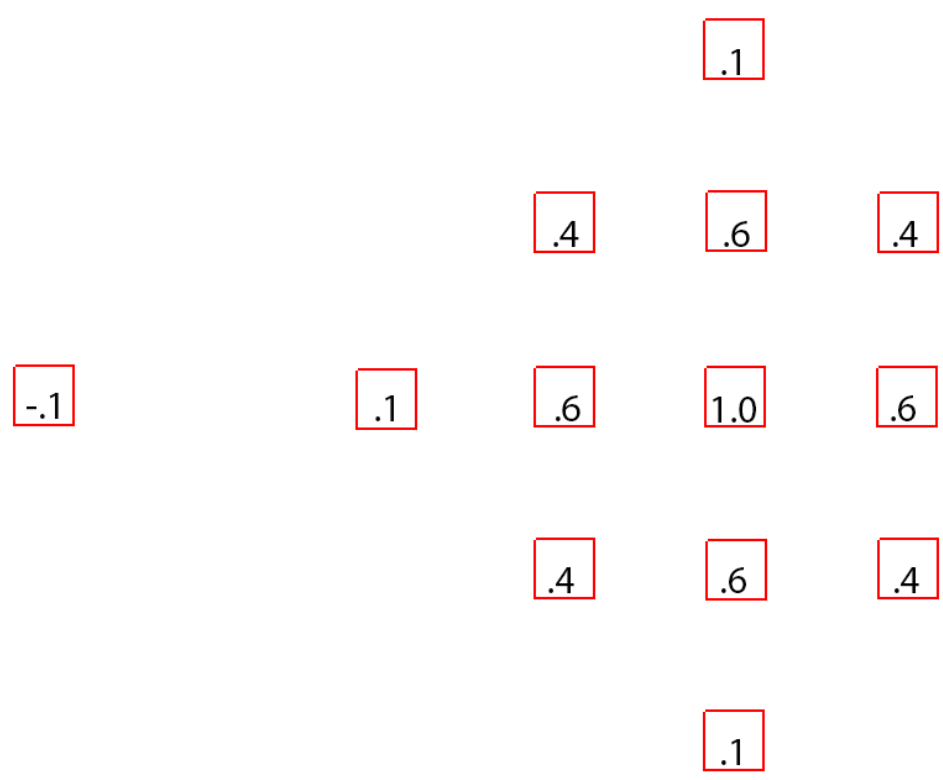

Figure 3.14: Weighting used for Gaussian distribution.

Theoretically, a Gaussian distribution in the near field would produce a Gaussian shape in the far field and with no sidelobes [17]. The results illustrated a slight reduction in sidelobe levels as well as a wider main beam when Gaussian weighting was applied to the sampled sinc patch configuration. The weighting of the squaring patches was swept from $0 \mathrm{~A}$ to $-1.0 \mathrm{~A}$ to determine which performed the best. The weighting of the elements from $0 \mathrm{~A}$ to $-0.4 \mathrm{~A}$ is shown below in Figure 3.15 . 


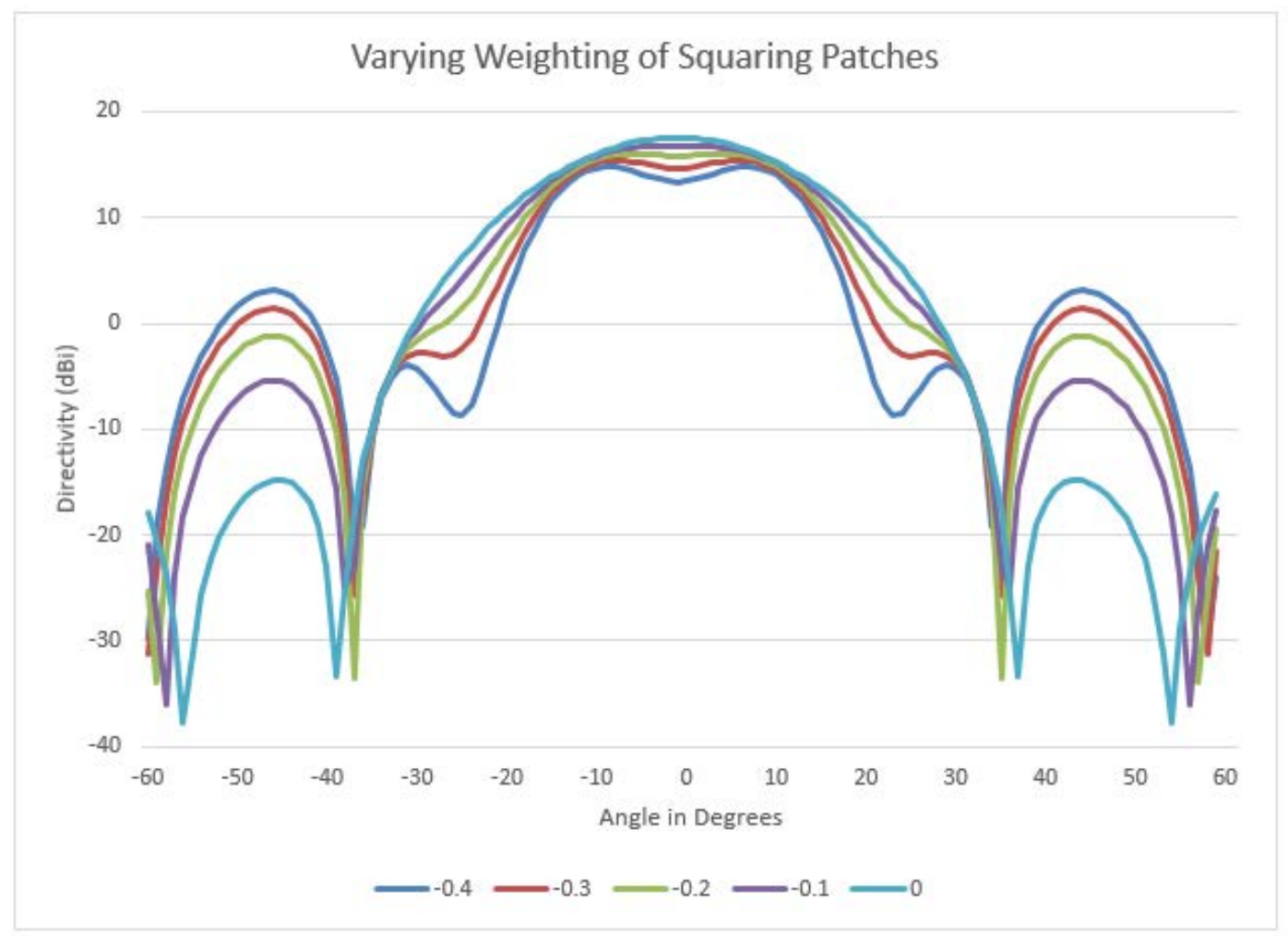

Figure 3.15: Comparison of different weightings of squaring elements.

The results show that without any power going to the squaring patches, it produces the lowest sidelobe levels, however it does not produce a square beam. Moving up in amplitude created an increase in squareness, but also an increase in sidelobe levels. In order to reduce sidelobe levels while still maintaining a square beam, a weighting of $-0.1 \mathrm{~A}$ was applied. This solutions works because the main beam of a sinc wave is extremely close to that of a Gaussian distribution. With the addition of the negative amplitude patches, the Gaussian distribution resembles a sinc function. The results are shown in Figure 3.16. 


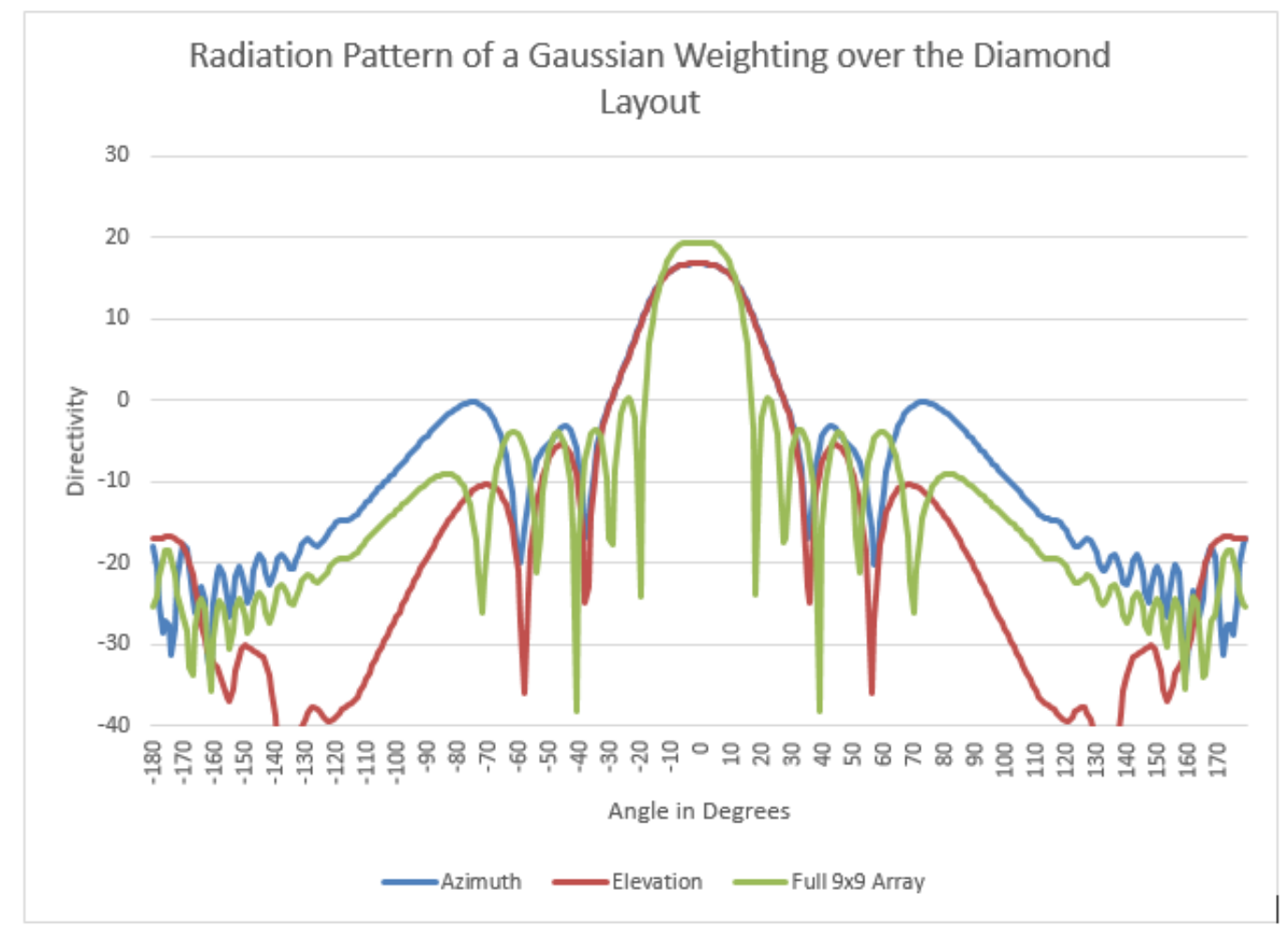

Figure 3.16: Radiation pattern of a Gaussian distribution over sinc layout.

However, the sidelobe levels are still too high in the results. The shape of the main lobe is also much wider: the applied Gaussian distribution is shown in the comparison with the full array pattern.

The azimuth and elevation patterns in this configuration were very different because of the asymmetrical feed in the $\mathrm{X}$ and $\mathrm{Y}$ plane on the patch. If the excitation was uniform, the field intensity on each patch would be symmetric and would have an effect on the sidelobe levels. In an attempt to reduce sidelobes and also allow for dual polarization of the patch, the feed of the patch was rotated 45 degrees. The transformation is shown in Figure 3.17. A second feed can then be placed in an adjacent corner that enables dual polarization that is required for MIMO. 

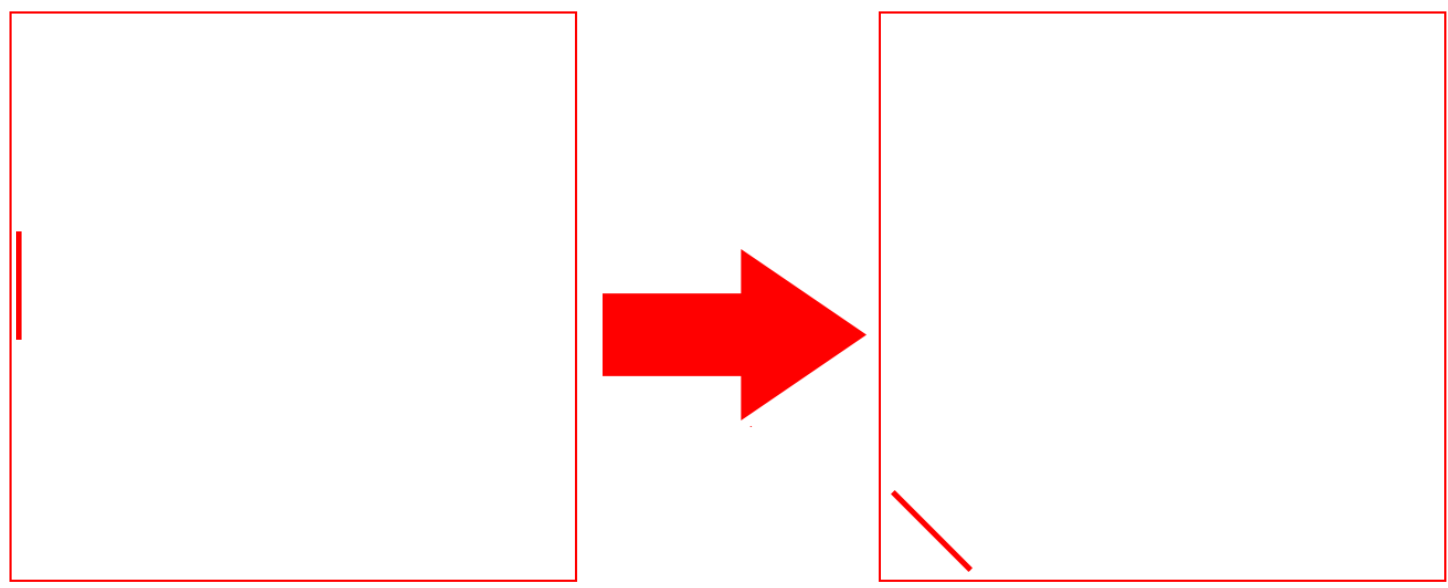

Figure 3.17: Location of the port feeding the patches.

The rotation of the ports slightly lowered sidelobe levels; however, more importantly, it resulted in the azimuth and elevation being equal and allowing for dual polarization and MIMO operation.

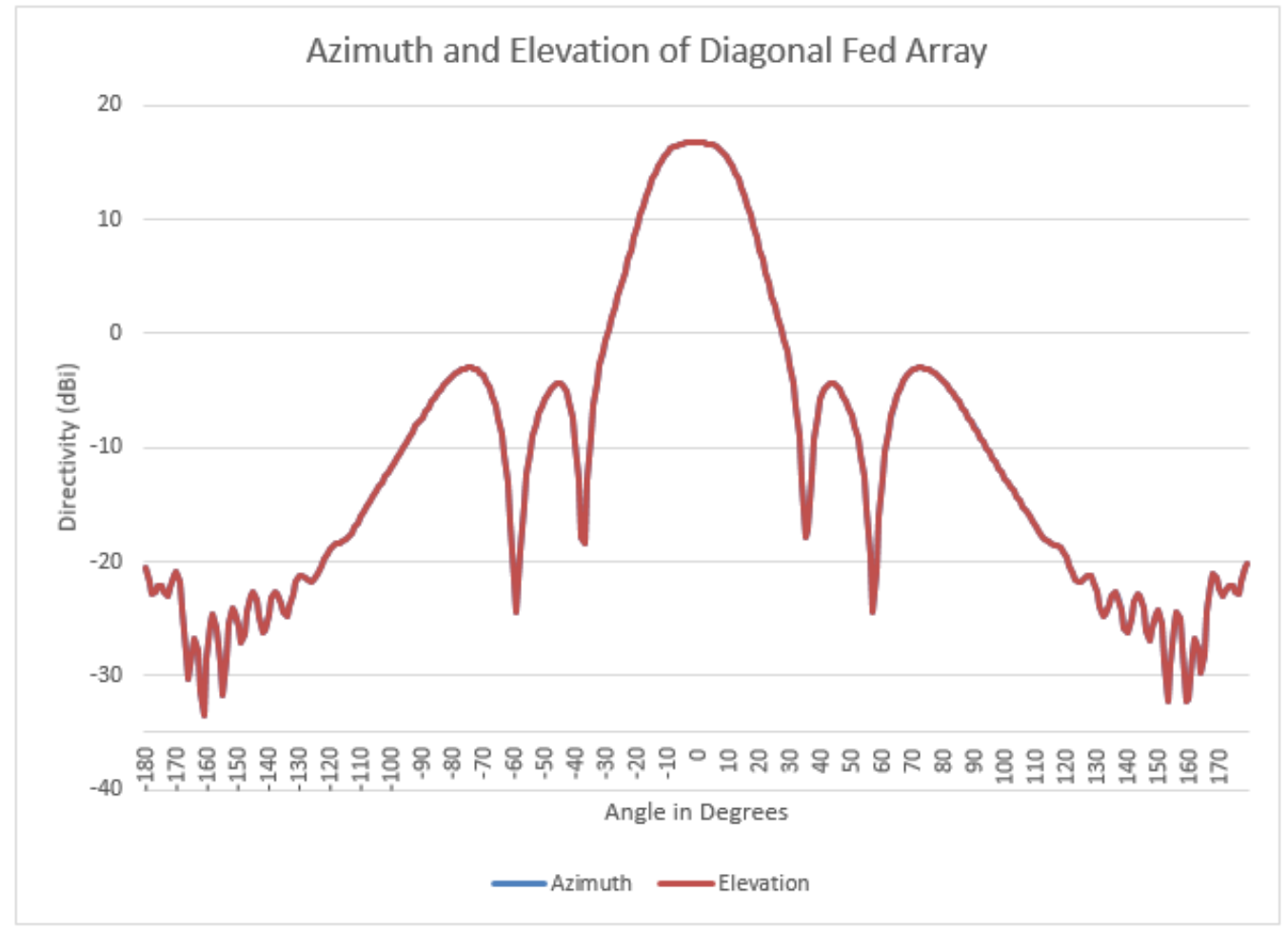

Figure 3.18: Radiation pattern with the rotated feed. 
The spacing between elements was reduced so that the grating lobes could be removed; however, it was at the expense of increasing the beamwidth. Spacing was then reduced by $0.1 \lambda$ to $0.6 \lambda$ producing the following results.

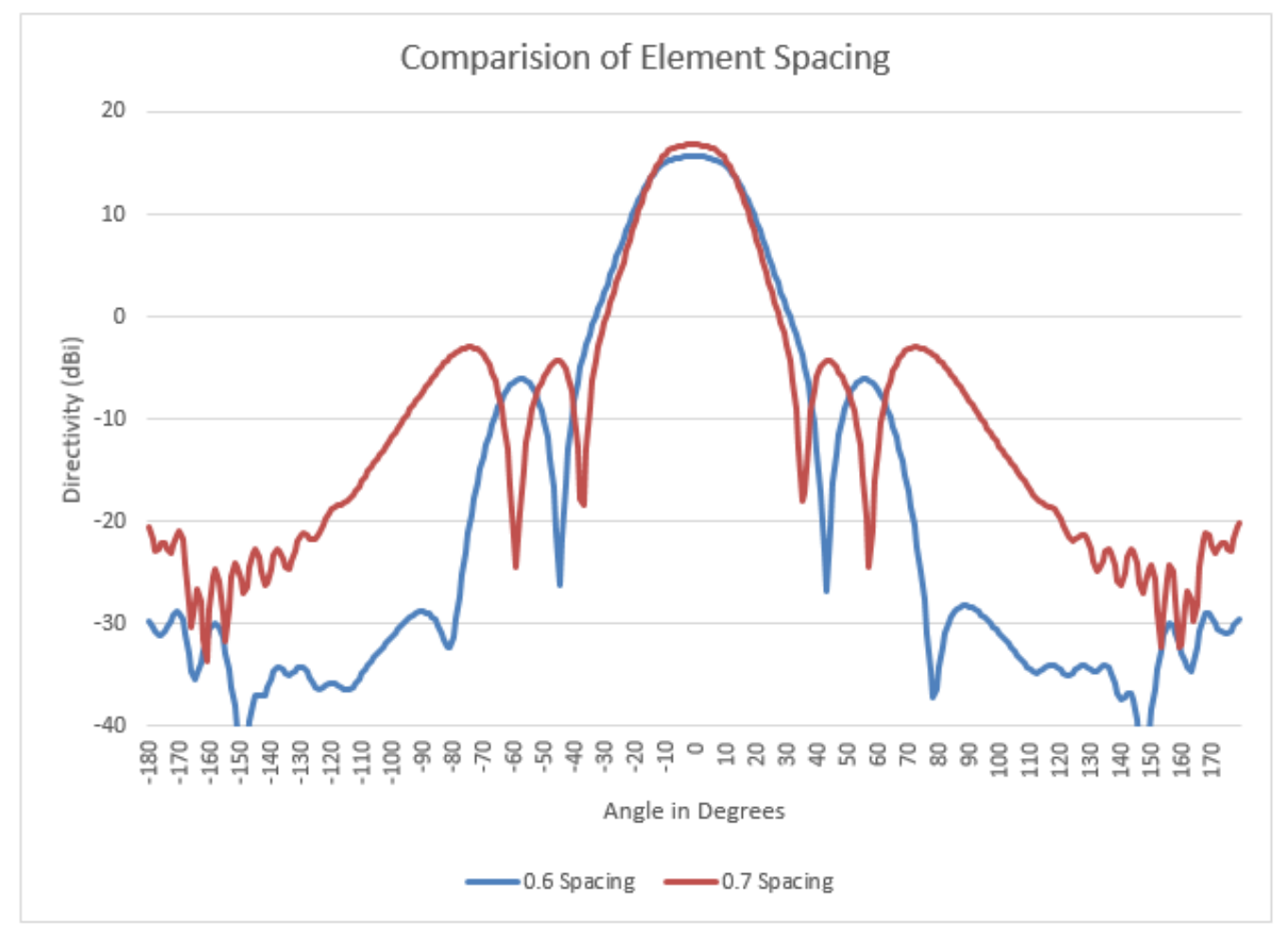

Figure 3.19: Radiation pattern with varying element spacing.

The results show there was a difference in the location and number of sidelobes. The change to $0.6 \lambda$ reduced the number of main sidelobes to one, and there were no minor sidelobes in its immediate vicinity. The disadvantage is that the aperture is reduced and the beamwidth increased from 27.4 degrees to 30.2 degrees. This reduction to one sidelobe allowed for the introduction of a sub array to cancel the lone sidelobes based on their location and phase. 


\subsection{Sidelobe Suppression}

The first sidelobes are typically 180 degrees out of phase from the main beam. An explanation of the sidelobes can be described using Digital Signal Processing theory [1]. A square window will produce a sinc function in the frequency domain. The sampled aperture of an array can be thought of as a square window that transforms into a sinc function. All arrays are of a finite size, they will always be mathematically windowed and sampled. Because the aperture of the array physically ends abruptly a square window can be assumed. There is also a secondary window based on the weighting of the elements as well as the shape of the array. When the rectangular window is considered by itself it is convolved with the sinc function in the near field.

This square window in the near field transforms into a sync function in the far field. The first sidelobe of the sinc function appears in the far field radiation pattern as the first sidelobe of the desired radiation pattern. This further explains why the first sidelobe has a 180 degree phase considering the first sidelobe of a sinc function is 180 degrees out of phase from the main lobe. The secondary windows from the Gaussian distribution helped shape and reduce the secondary sidelobes while the first sidelobes remained.

The windowing effect can also be used to explain why the beam of an equally weighted uniformly spaced array is elliptical. Without regards for the radiation pattern of the patch, the array factor still produces an elliptical beam because the discrete time Fourier transform is the same function as the array factor. Since everything is finite, a rectangular window can be assumed. This rectangle window transforms into

a sinc function in the far field. A radiation pattern shows the magnitude: the sinc function would be squared producing the sidelobes as seen in a regular radiation pattern. The phases of each lobe alternates as in a sinc function. 
This is illustrated in the angle of the radiation pattern of the array in Section 3.5, as shown in Figure 3.20. The figure shows the sidelobes and how the phase changes at each sidelobe.

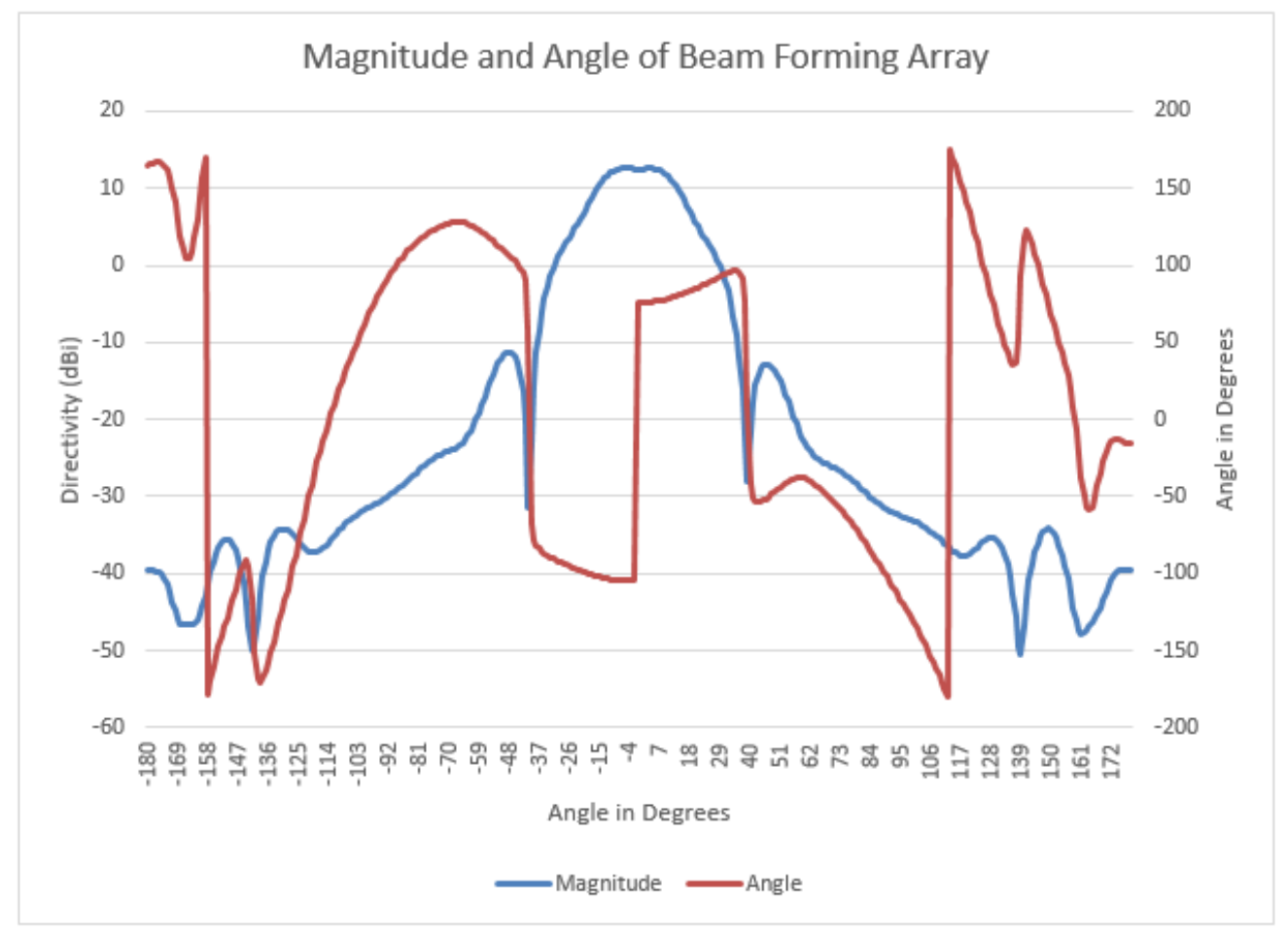

Figure 3.20: Radiation pattern with phase of the beam forming array.

Location, weighting, and phase of the patches are all explored next. The patches were initially placed 45 degrees away from the center of the array. This allowed symmetry in the $\mathrm{X}$ and $\mathrm{Y}$ planes in the array, and that would mean symmetry in the far field in elevation and azimuth. The spacing from the array was in line with the grid formed by the remaining patches, creating a foundation to start optimization. The side lobe suppressing sub array patches were fed with a phase opposite that of the 180 degree sidelobe: a phase of 0 degrees. The amplitude fed to the patches was a current of $0.1 \mathrm{~A}$, chosen because the current at adjacent patches was also $0.1 \mathrm{~A}$ and it provided a starting point for optimization. The radiation pattern of the suppressing 
sub-array is important because the resultant sidelobes are what provide suppression. The location of the suppressing sidelobes can be found by plotting the array factor of a $2 \times 2$ array with large spacing. By simulating the sidelobe suppressing sub array by itself the radiation pattern is found as shown in Figure 3.21.

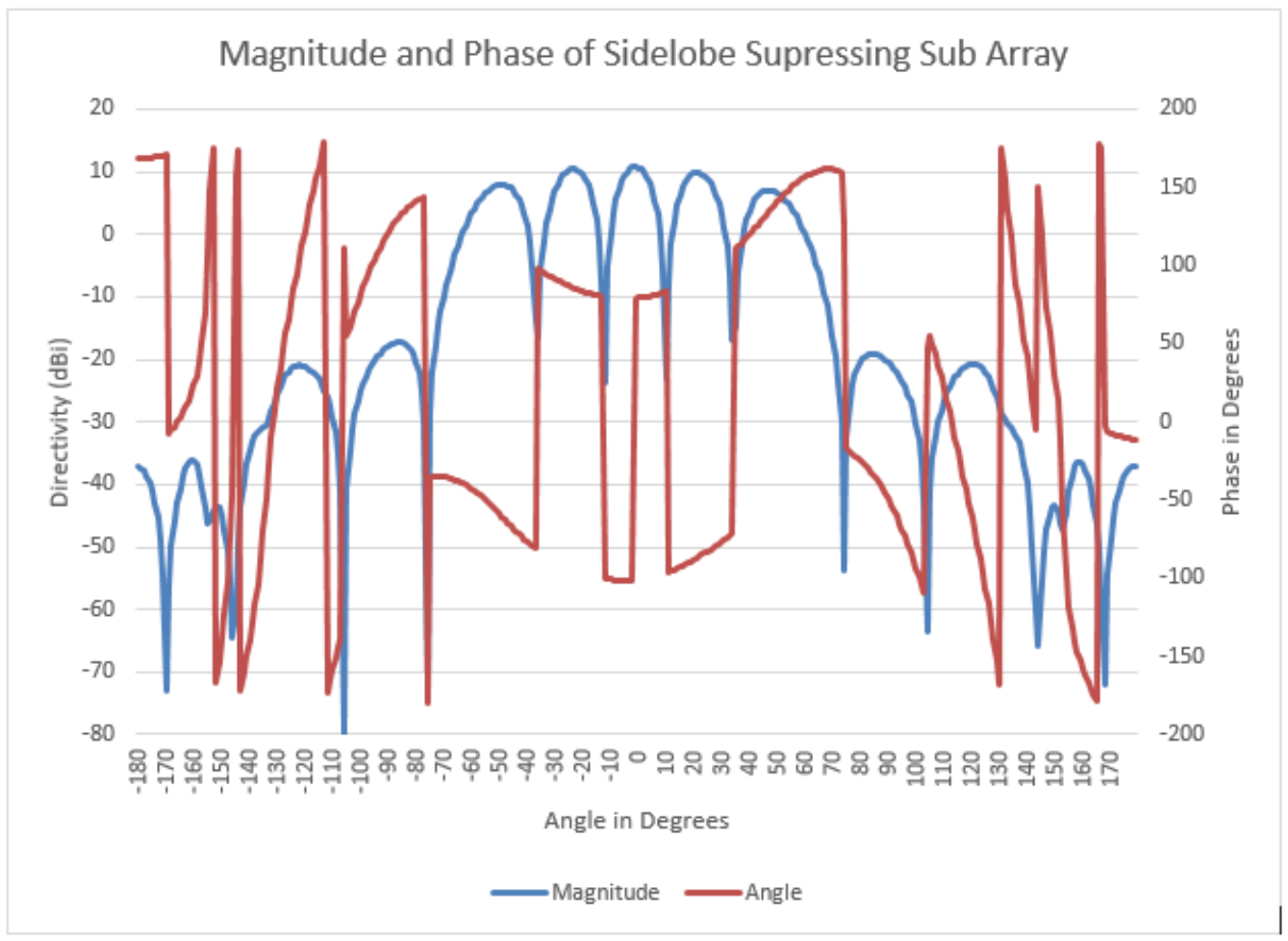

Figure 3.21: Radiation pattern with phase of the sidelobe supressing sub array.

In order for cancellation to occur, a sidelobe of opposite phase needs to be in line with the sidelobe from the beam forming sub array radiation pattern as indicated in Figure 3.21. The phase of the third lobe is opposite that of the second lobe in the beam forming array. The position of the sidelobe cancelling patches were in and out along the 45 degree axis to investigate sidelobes movement. These results are seen in Figure 3.22, where moving the patches out caused the sidelobes to move in. This control allows for the sidelobe to be placed directly in line with the sidelobe in the 
beam forming array requiring suppression: this corresponds to $0.15 \lambda$ away from the center along the 45 degree axis.

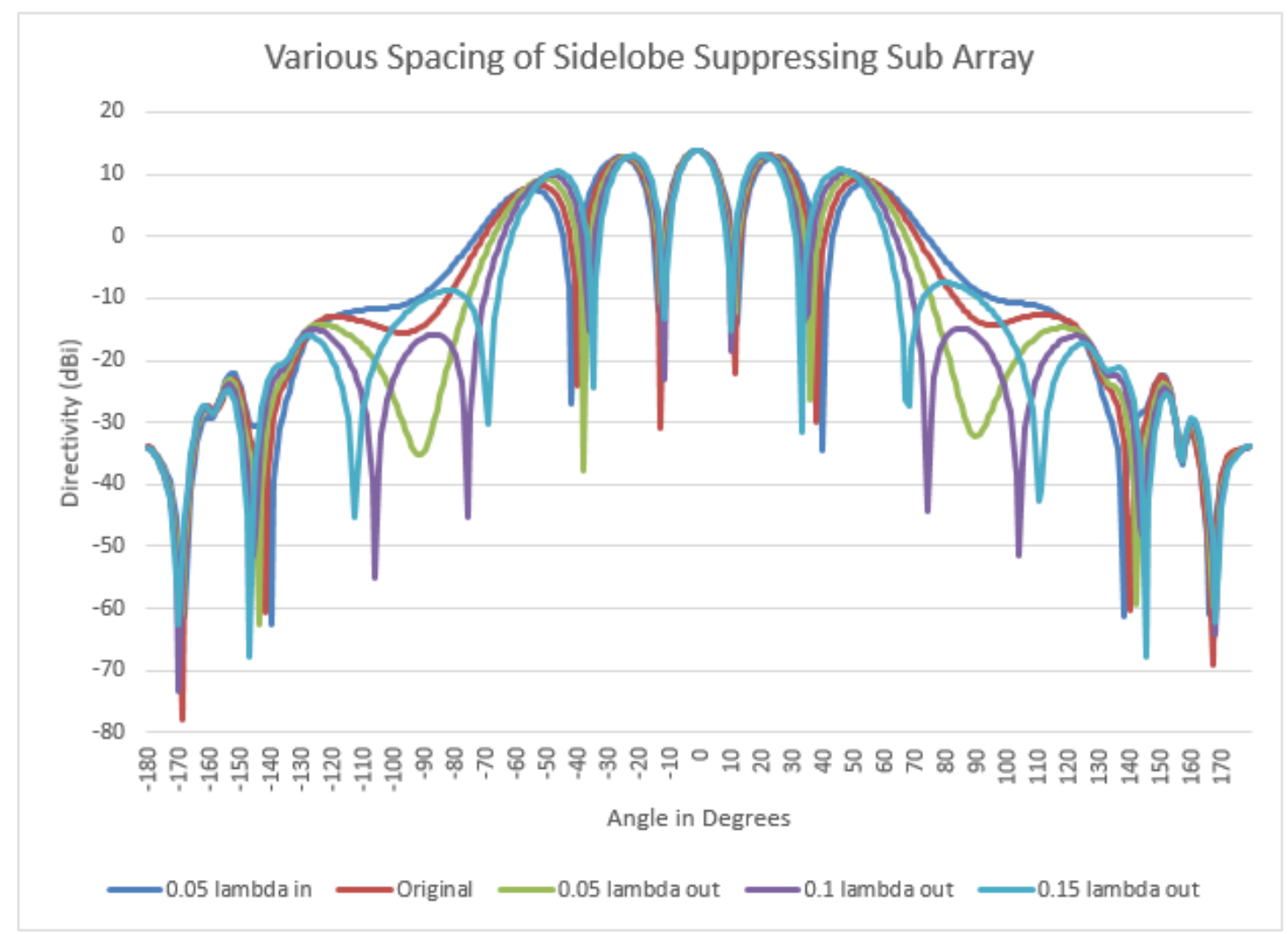

Figure 3.22: Radiation pattern of the sidelobe supressing sub array at different positions.

After determining the ideal location (sidelobes being at 46 degrees as illustrated in Figure 3.19 in Section 3.5), the sidelobe suppressing elements were placed in the full array and simulated. In order to confirm that they were in the correct location, the same parameter sweep was done to determine the location. The results in Figure 3.23 demonstrate that while placing the suppressing patches $0.15 \lambda$ out produced the lowest first and second side lobe levels the third sidelobe sidelobe is higher than all sidelobes at $0.1 \lambda$ away. 


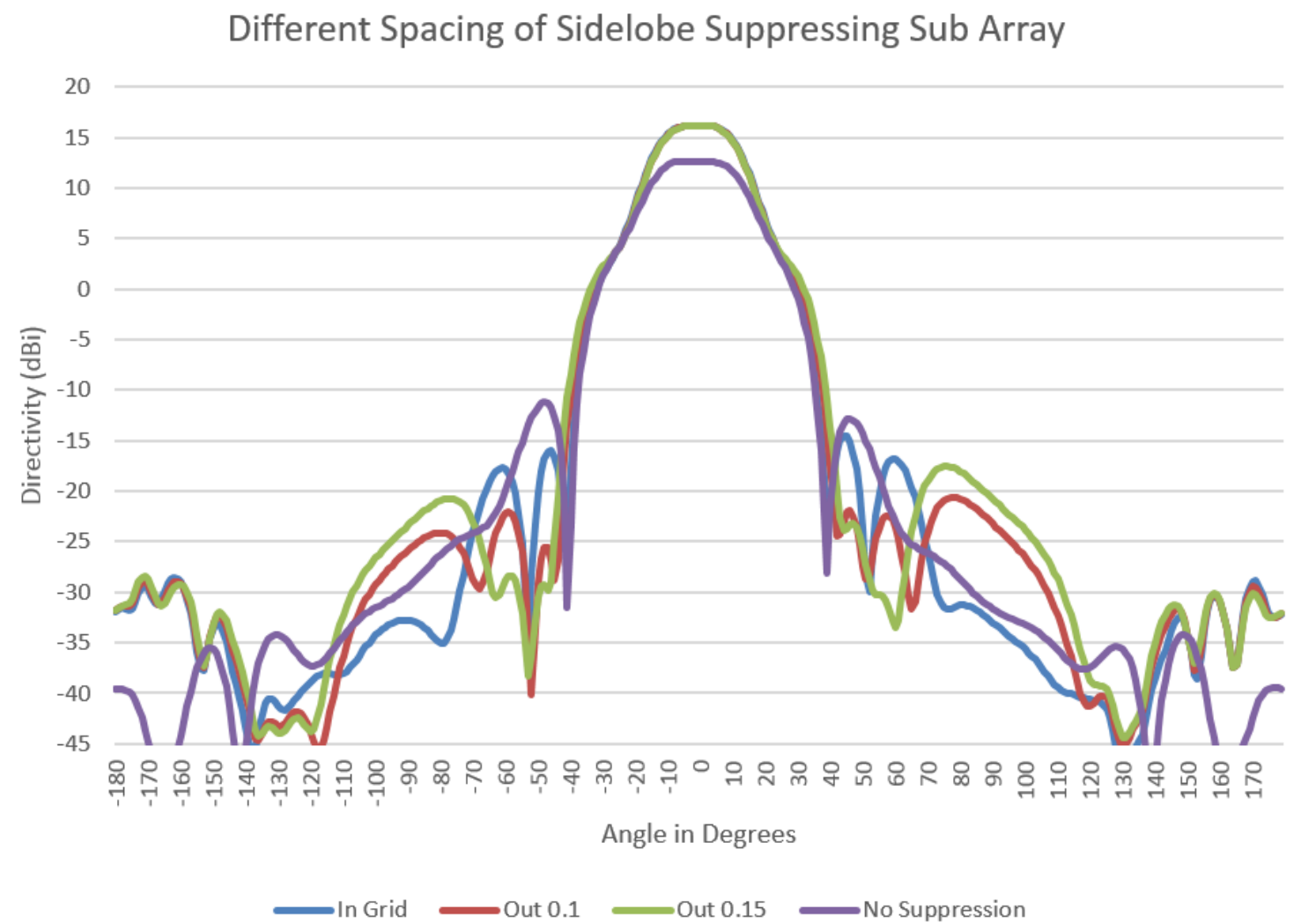

Figure 3.23: Radiation pattern of the full array with suppressors at different positions.

Even if the sidelobe suppressing elements are not in the right location, the presence of the sidelobe suppressing elements can reduce the sidelobe. The results show a drop in SLL from $-25 \mathrm{~dB}$ to $-32 \mathrm{~dB}$ with the sidelobes placed in the same grid as the array, and a further reduction to $-34 \mathrm{~dB}$ when optimized using a $0.15 \lambda$ shift, and $-37 \mathrm{~dB}$ using a $0.1 \lambda$ shift out. The third sidelobe of the suppressing element sub array is greater at $0.15 \lambda$ shifted, and therefore has a greater effect pulling the sidelobe higher than all the $0.1 \lambda$ shifted sidelobes.

The optimal location of the sidelobe suppressing subarray depends on application. If the lowest possible first sidelobe is desired to be beside the main lobe then the $0.15 \lambda$ shifted spacing is better. However, if lower overall sidelobes are more desirable then 
the $0.10 \lambda$ shifted spacing are used.

With the optimized location determined for this array, the weighting of the elements were optimized next. As before, it is difficult to determine the power at the sidelobe that would be doing the canceling without simulating or plotting the equations. The results of sweeping from a weighting of $0 \mathrm{~A}$ (no suppressing patch) to a weighting of $0.25 \mathrm{~A}$ with increments of $0.05 \mathrm{~A}$ are seen in Figure 3.24.

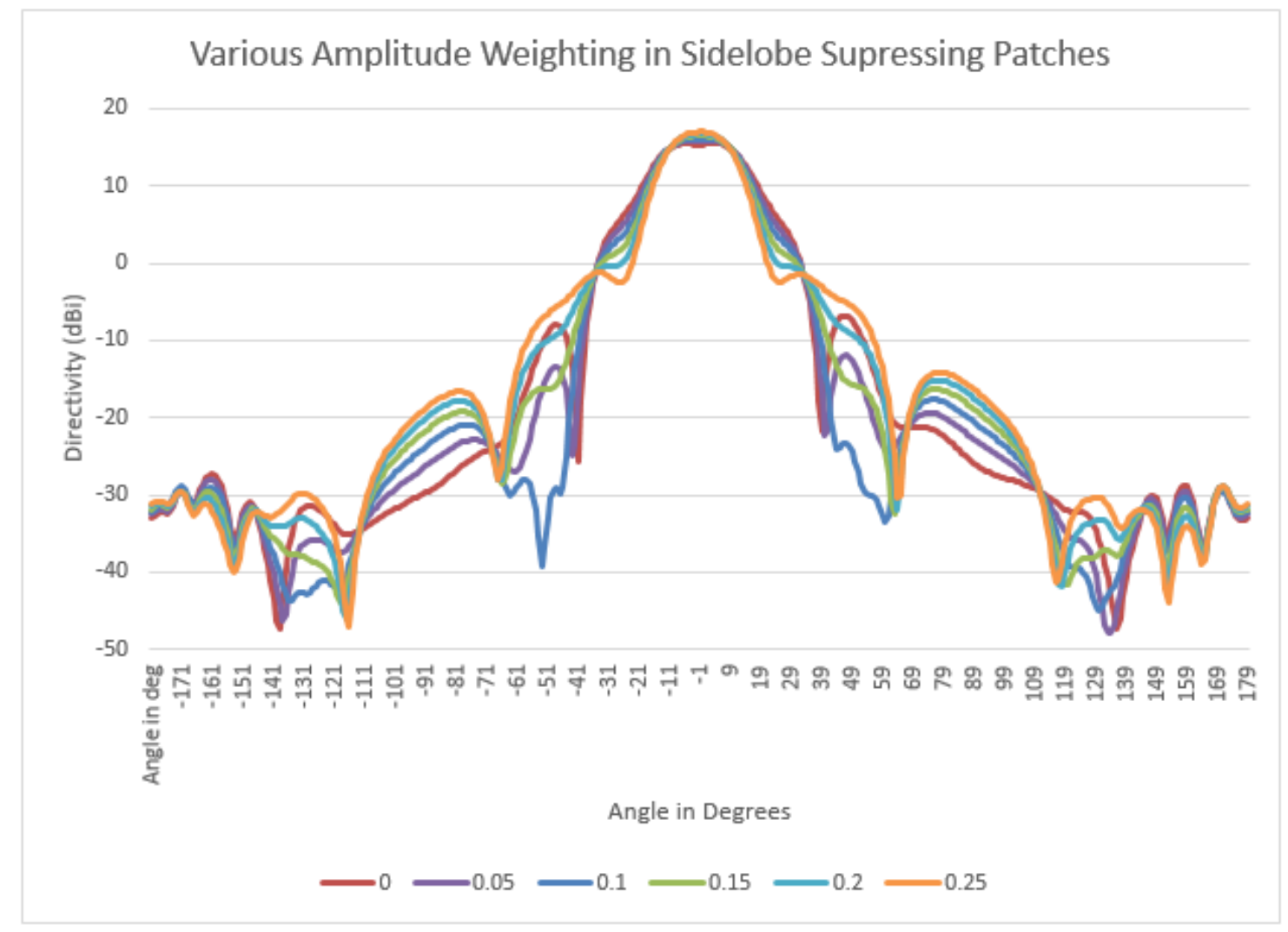

Figure 3.24: Radiation pattern of the full array with various weightings.

These results show that a weighting of $0.1 \mathrm{~A}$ does provide the optimal results. However all weightings between $0.5 \mathrm{~A}$ and $0.2 \mathrm{~A}$ do cause a SLL reduction when compared to the case with no patchs. When the power in the suppressing sidelobe is too large, the phase of the sidelobe from the beam forming array was switched from 
180 to 0 , because the positive component from the suppression would dominate and cause the sidelobe to rise. This is why the second sidelobe in Figure 3.26 rises; the power in the 4th sidelobe of the suppressing elements is larger than that of the beam forming arrays sidelobe level. The power level in that suppression sidelobe dominates the array's original second sidelobe.

The layout of the suppressors on the array are shown in Figure 3.25 and the far field results in Figure 3.26.

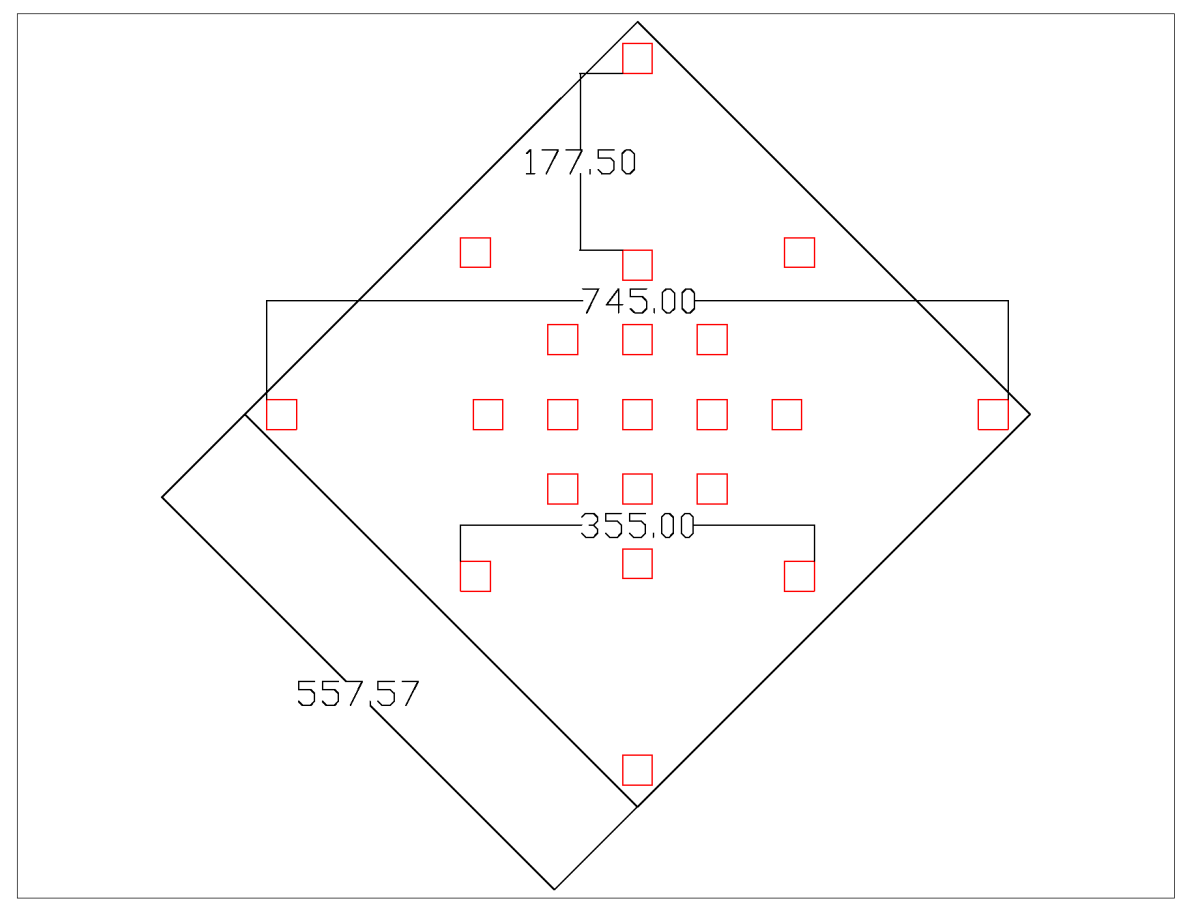

Figure 3.25: Dimensions of full array.

These sidelobe levels are now below the goal of $-30 \mathrm{~dB}$ and the dimensions of the array also fit as shown in the layout below. The specification was $600 \mathrm{~mm} \times 600 \mathrm{~mm}$. While it does not fit when oriented as a square with blank spaces, it does fit when 
rotated as a diamond with a size of $557.57 \mathrm{~mm} \times 557.5 \mathrm{~mm}$.

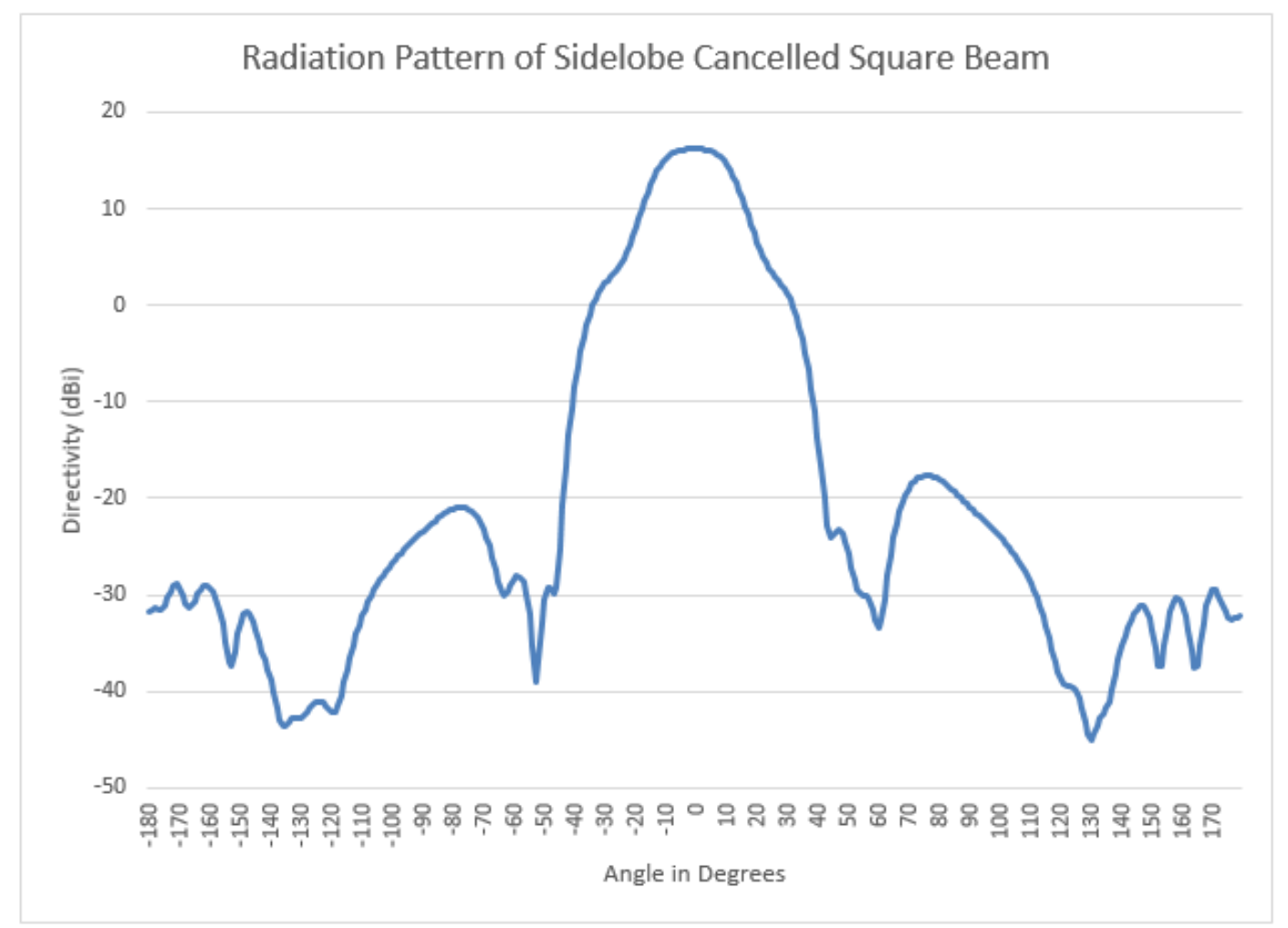

Figure 3.26: Radiation pattern of full array.

This figure shows azimuth, elevation, and the 45 degree cut. The azimuth and elevation cuts are identical; however, the 45 degree cut is different. The half power beam width is wider then the azimuth and elevation. The 45 degree axis of a square is longer then any side by a factor of $\sqrt{(2)}$.

\subsection{Ultra Low Sidelobe}

A Gaussian weighting was tested without the outer inverted weighted patches to determine the performance of a smaller form factor antenna. The layout is simply the inner diamond being fed with the exact same weighting as above, without using 
the outer patches.
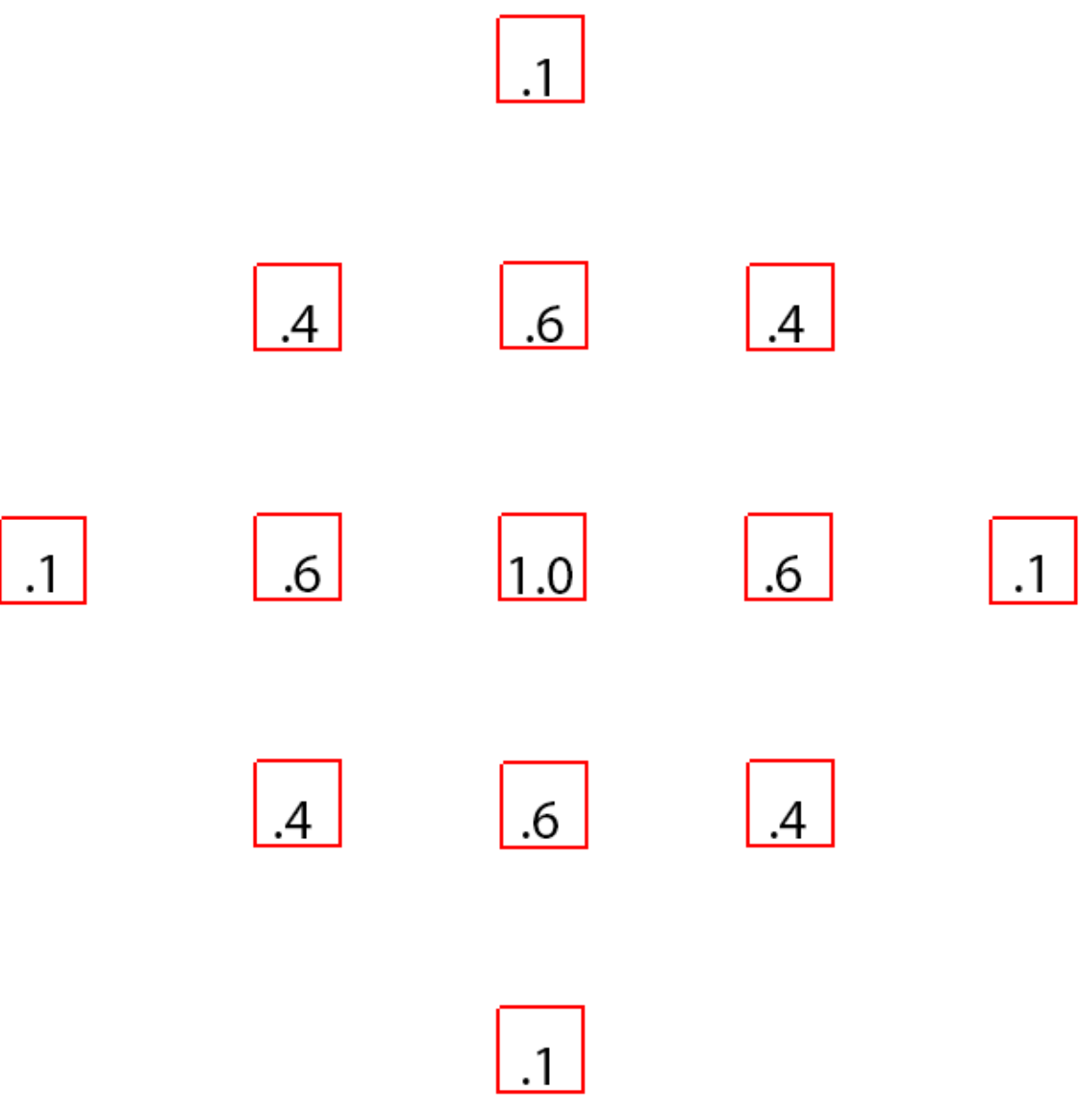

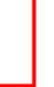

.

$$
\text { a }
$$




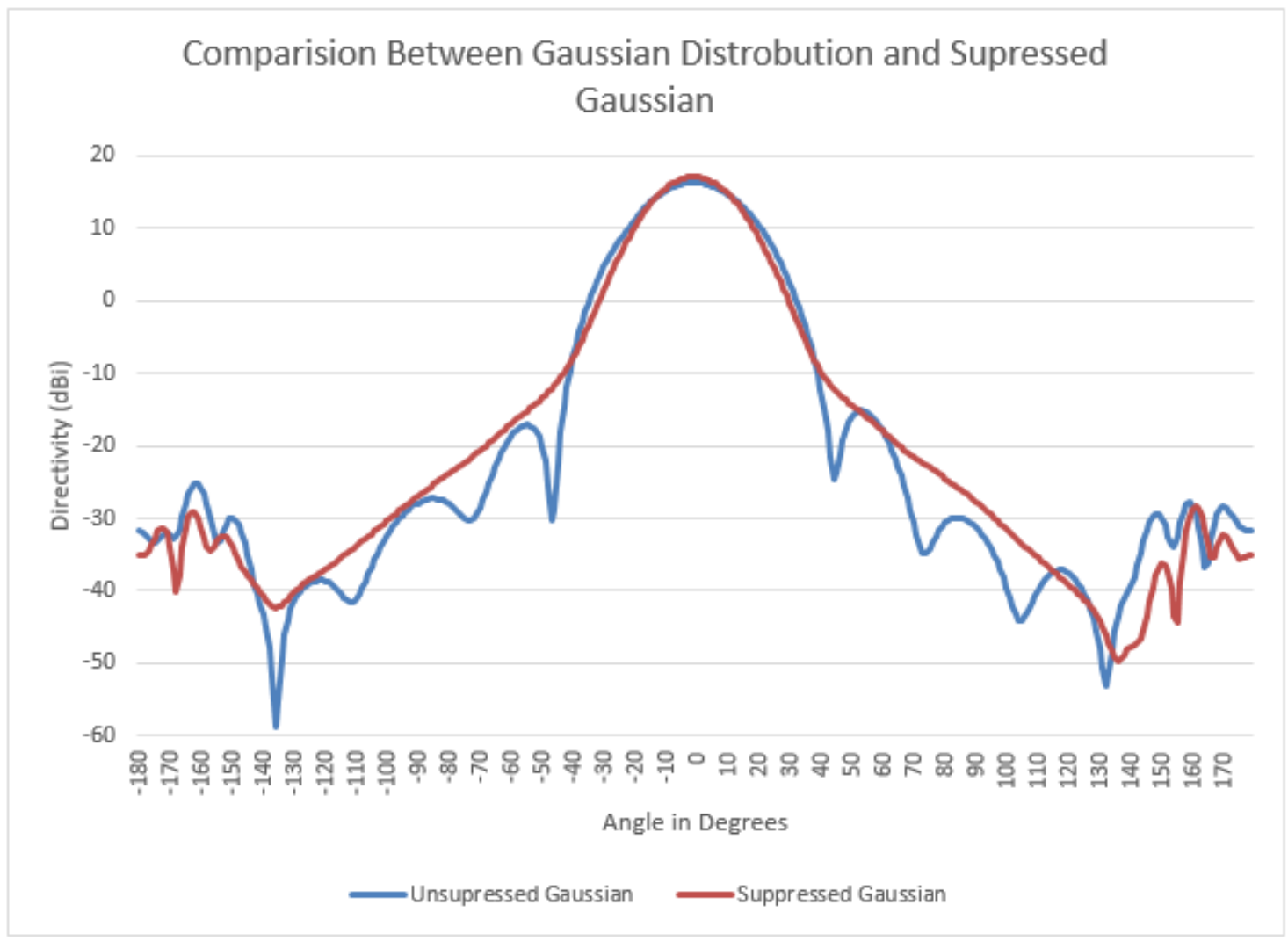

Figure 3.28: Comparison between Gaussian distributed with and without suppressing sub-array.

The sidelobe suppressing elements were placed in the same location as the square beam as the sidelobes of the Gaussian beam forming array were in the same position as the square beam. The results of a simulation with sidelobe suppressing patches added in the same position as in Section 3.6, was a radiation pattern with practically no sidelobes at all. The roll off also coincides with the peaks of the sidelobes, however there are no direct sidelobes. In this case, the first side lobe is in the back lobe, and has a value of $-46 \mathrm{~dB}$.

Following the same methodology as above, the sidelobe suppressing elements were shifted along the 45 degree axis from the center. The optimal location was the same as in Section 3.6 given the sidelobes are in the same position. This had the effect of 
lowering the energy at about 45 degrees by $10 \mathrm{~dB}$ to below $-40 \mathrm{~dB}$ from the main lobe. While simulation results of an array with sidelobe levels of -40dB are not impossible, they are rare - especially with a wide beamwidth in comparison to the results in literature [18-20].

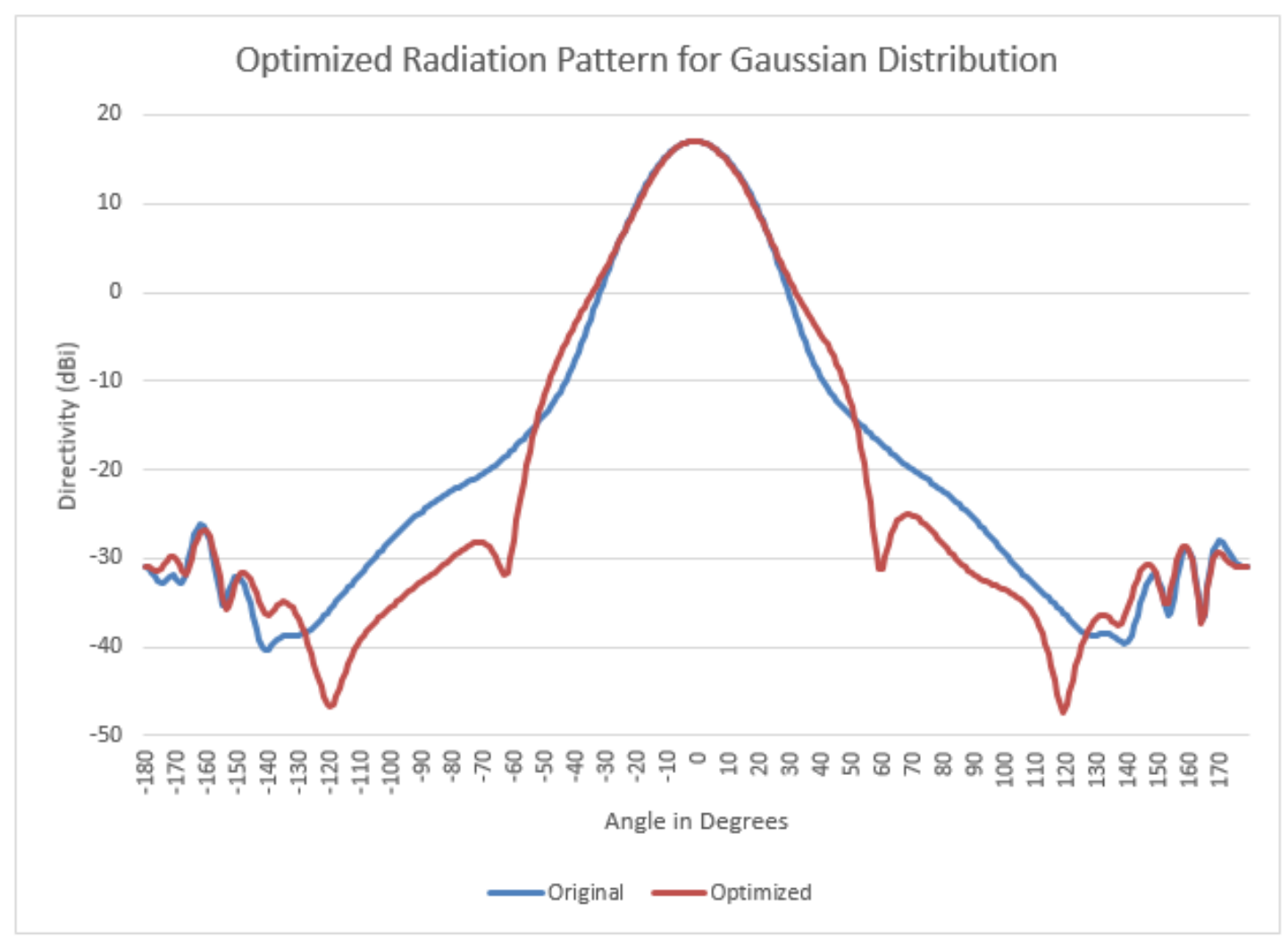

Figure 3.29: Comparison between optimized position and non-optimized. 


\subsection{Summary of Results}

Table 3.1: Summary of Results.

\begin{tabular}{ccccc}
\hline Layout & $\begin{array}{c}\text { Directivity } \\
(\mathrm{dBi})\end{array}$ & $\begin{array}{c}\text { Max Sll } \\
(\mathrm{dB})\end{array}$ & $\begin{array}{c}\text { Beam Width } \\
(\text { Degrees })\end{array}$ & $\begin{array}{c}\text { Size } \\
(\mathrm{mmXmm})\end{array}$ \\
\hline \hline 9x9 Full Array & 19.4 & 19.1 & 21.5 & $730 \times 730$ \\
Equal Weighted & 16.4 & 19.2 & 25 & $557.57 \times 557.57$ \\
Gaussian Weighted (Square) & 16.8 & 17 & 27.3 & $557.57 \times 557.57$ \\
Rotated Feed & 15.6 & 26.1 & 30.4 & $557.57 \times 557.57$ \\
With Sidelobe Suppression & 16.2 & 30.8 & 27.4 & $557.57 \times 557.57$ \\
Optimized Location (1) & 16.2 & 33.8 & 26.8 & $557.57 \times 557.57$ \\
Optimized Location (2) & 16.2 & 36.8 & 26.9 & $557.57 \times 557.57$ \\
Unsuppressed Gaussian & 16.3 & 33.4 & 30 & $330 \times 330$ \\
Optimized Gaussian & 17 & 42.1 & 24.6 & $367.5 \times 367.5$ \\
\hline
\end{tabular}

\subsection{Conclusions}

These initial results indicate that the sidelobe goal was, in theory, possible to achieve and to exceed. The main specifications that were explored in the design, were the sidelobe levels, beamwidth, shape, and size of the array. The results above show the compromise between the beam width and size of the antenna. The size of the board is more important. The sidelobe levels surpassed expectations and the shape of the radiation pattern was square. The initial target gain of $18 \mathrm{dBi}$ was not attainable, except in the 9x9 planar array.

The next section covers the design of the feed network used to deliver the correct power and phase to all patches. The weighting and layout used in the following section is the square array with sidelobe suppression. 


\section{Chapter 4}

\section{Feed Network and System Design}

\subsection{Introduction}

A feed network was designed in order to provide the correct power and phase to each patch of the array. With the exception of the outer squaring patches that needed to be 180 degrees out of phase, the phases going to all of the patches needed to be the same. A number of power dividers needed to be designed in order to split the power correctly. The Wilkinson divider is used in the final design. While the TJunction was used in several earlier iterations.

\subsection{Patch Design}

The simulations completed in Chapter 3 were used as first order approximations for the array factor. A new patch had to be used to move towards a manufacturable design. The patch shown in Figure 4.1 was designed with four equal feeds in a push/pull configuration: the feeds opposite of each other are fed 180 degrees out of phase. The patch layer is separated by an air gap and is on 20 thou of FR4. The feed layer substrate is Rogers RO4003 with a sub array on top followed by 20 thou of 
RO4003 below. A copper ground plane with slots is followed by 20 thou of RO4003 with 4 thou of RO4450F PREPREG, and finally the feed layer at the bottom. This stack up can be seen in Figures 4.2.

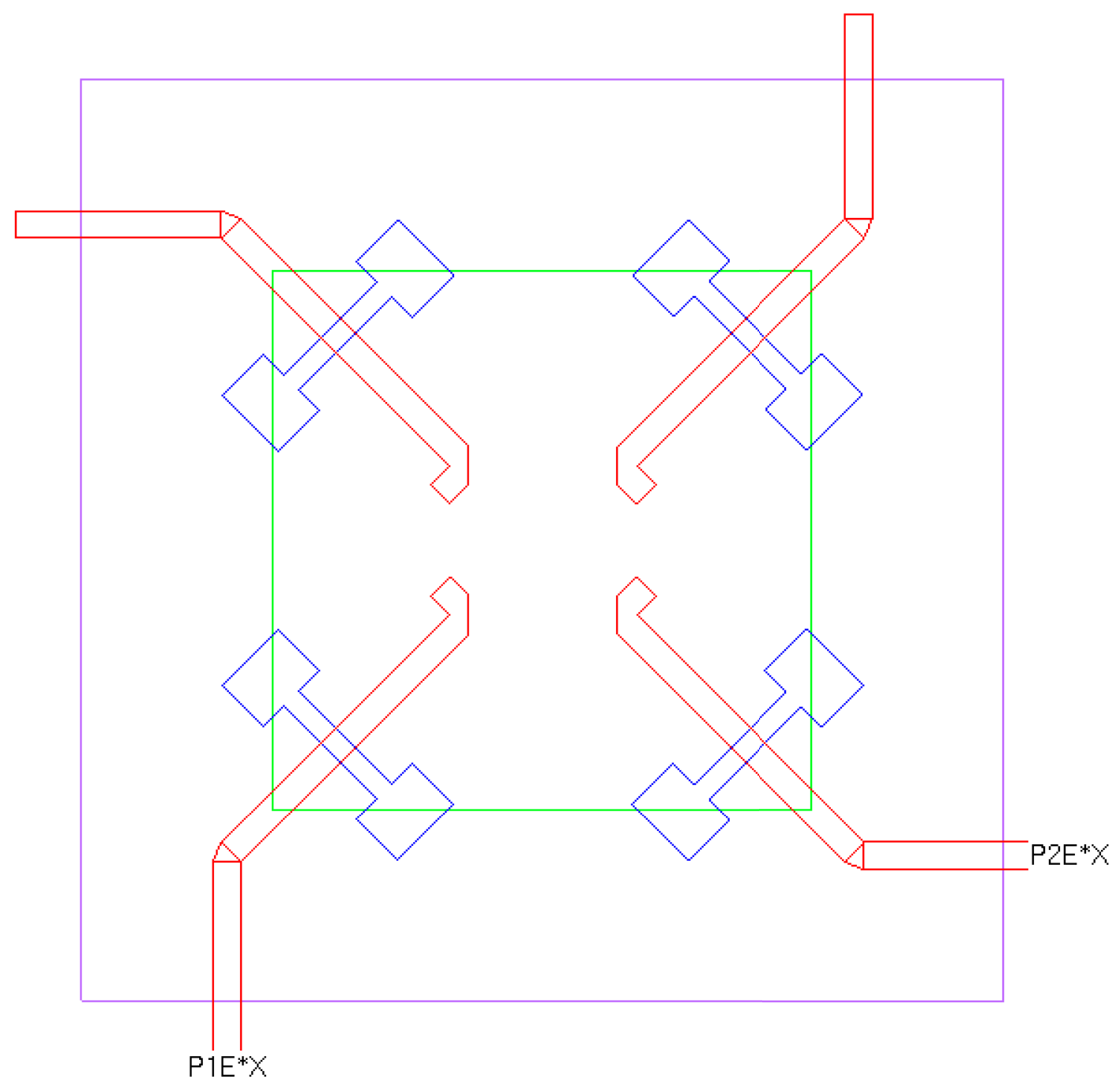

Figure 4.1: Layout of the microstrip patch. 


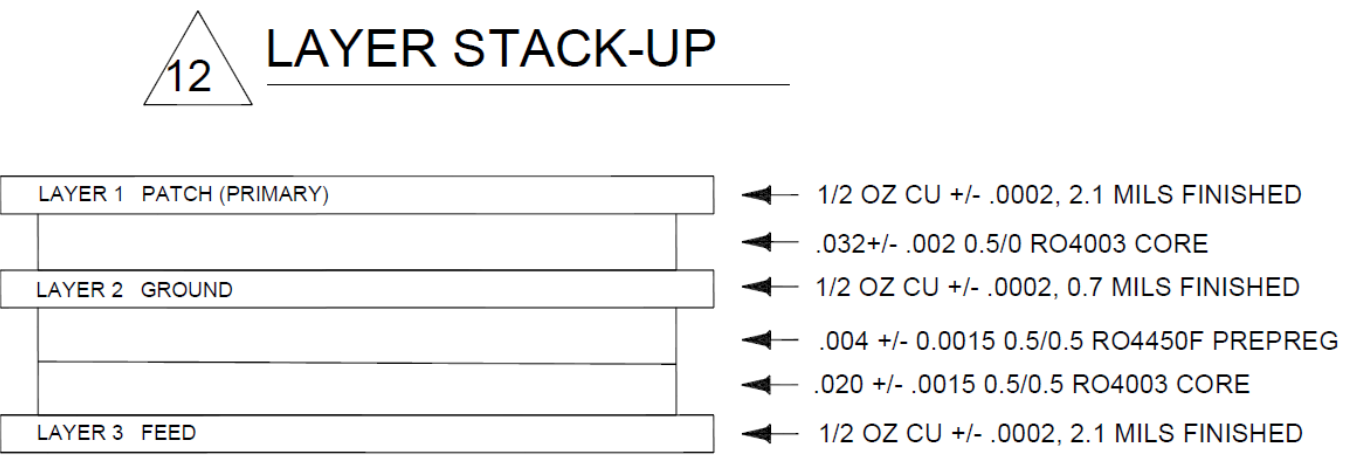

Figure 4.2: Stack up of array.

The isolation of the feed and the patch layers help to reduce coupling with the feed network and patches, which in turn, improves the return loss of the array. The gap between the FR4 and RO4003 helps to improve the bandwidth of the array because of the change in dielectrics.

Using two adjacent feeds with different inputs would allow the use of MIMO with the two polarizations as shown in the diagram in Figure 4.1. The red lines indicate the feed on the bottom layer and the slots in the ground plane are in blue. The green lines are the sub array on the top layer of the RO4003 and the purple layer is the top patch on FR4. One half the feed network is fed to the port labeled P1E*X, while $\mathrm{P}^{2} \mathrm{E}^{*} \mathrm{X}$ is fed to allow MIMO.

While the patches were initially designed for the push/pull configuration, it was not implemented due to the added complexity of the feed network and the minimal performance difference as illustrated in Figure 4.3 


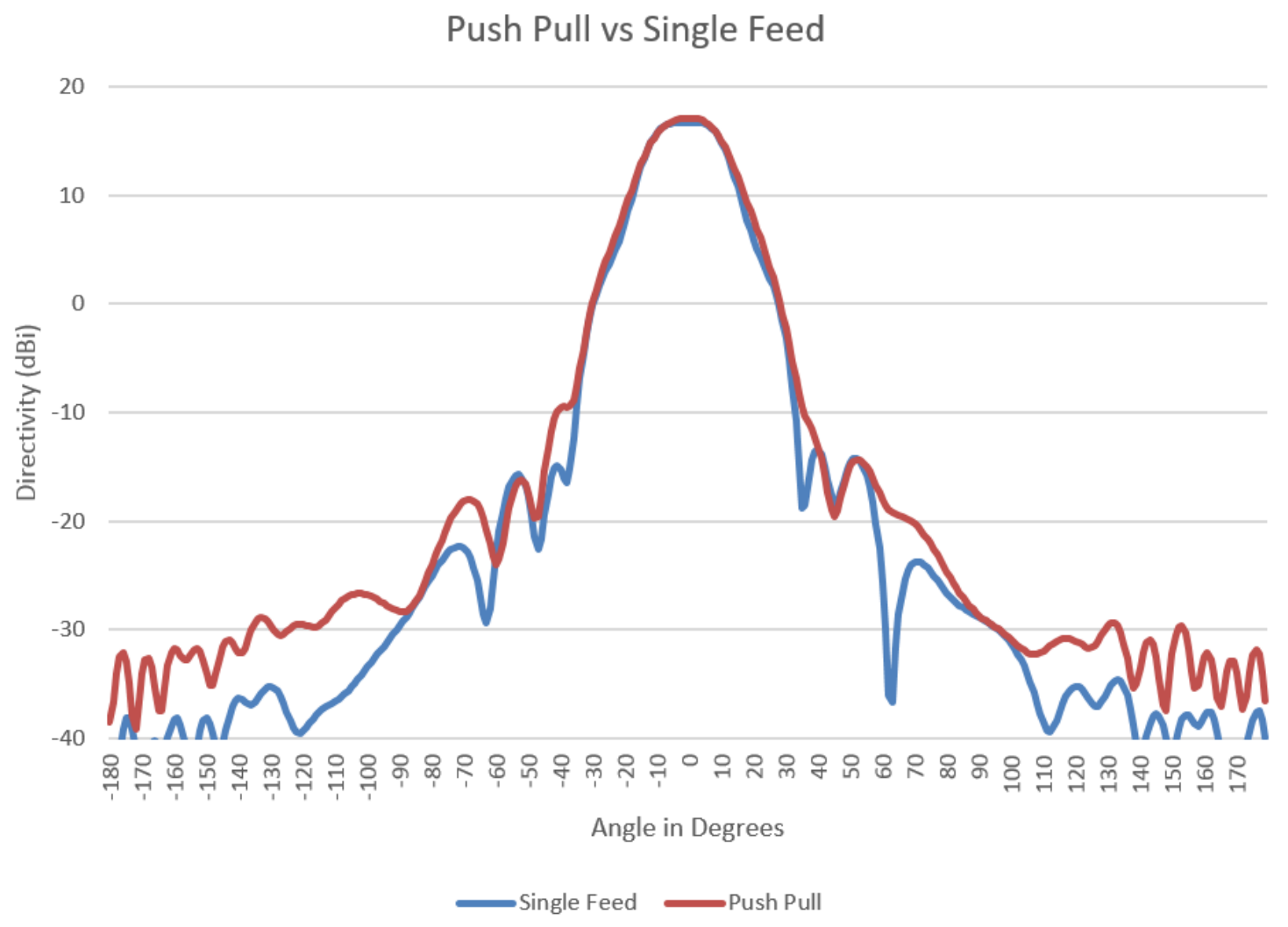

Figure 4.3: Difference between single feed and push/pull feed.

The patches were laid out in the same configuration in Figure 3.25 and the far field results shown in Figure 4.4 are overlaid with the original patch results. The results vary slightly around the sidelobes; however the peaks are the same. 


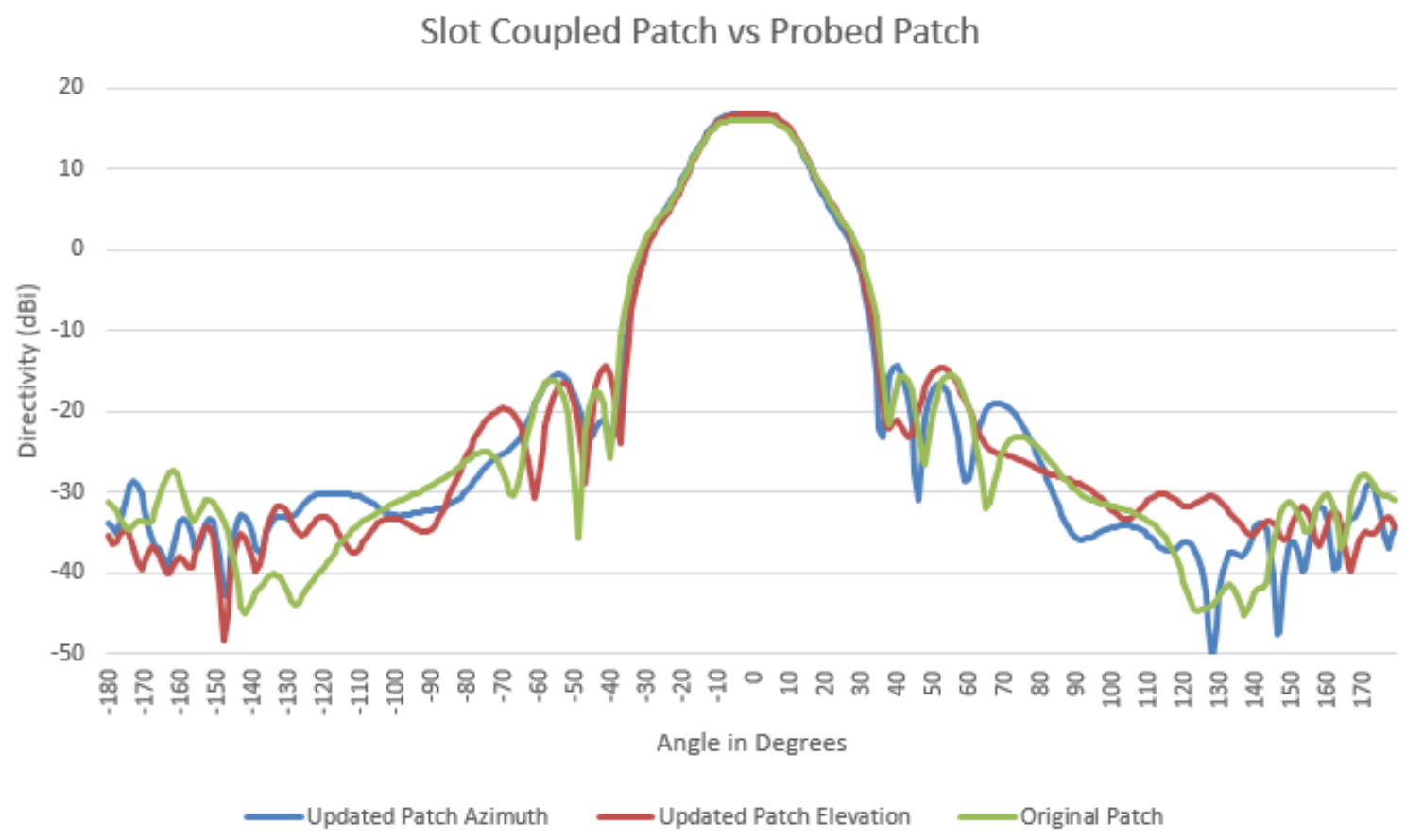

Figure 4.4: Comparison of slot fed patch and probed patch from Section 3.

\subsection{Feed Network Design}

The primary concern of the feed network itself is to ensure the delivery of the correct power and phases to excite each patch. One method of reducing the complexity is to locate a plane of symmetry as that would allow one half of the network to be identical as the other. The nature of the unequal weighting and the center patch being the only patch receiving a weighting of $1.0 \mathrm{~A}$, resulted in the symmetry shown below in Figure 4.5. The weightings in this section are power weightings expressed as a ratio between the center patch and the weighting being examined. 


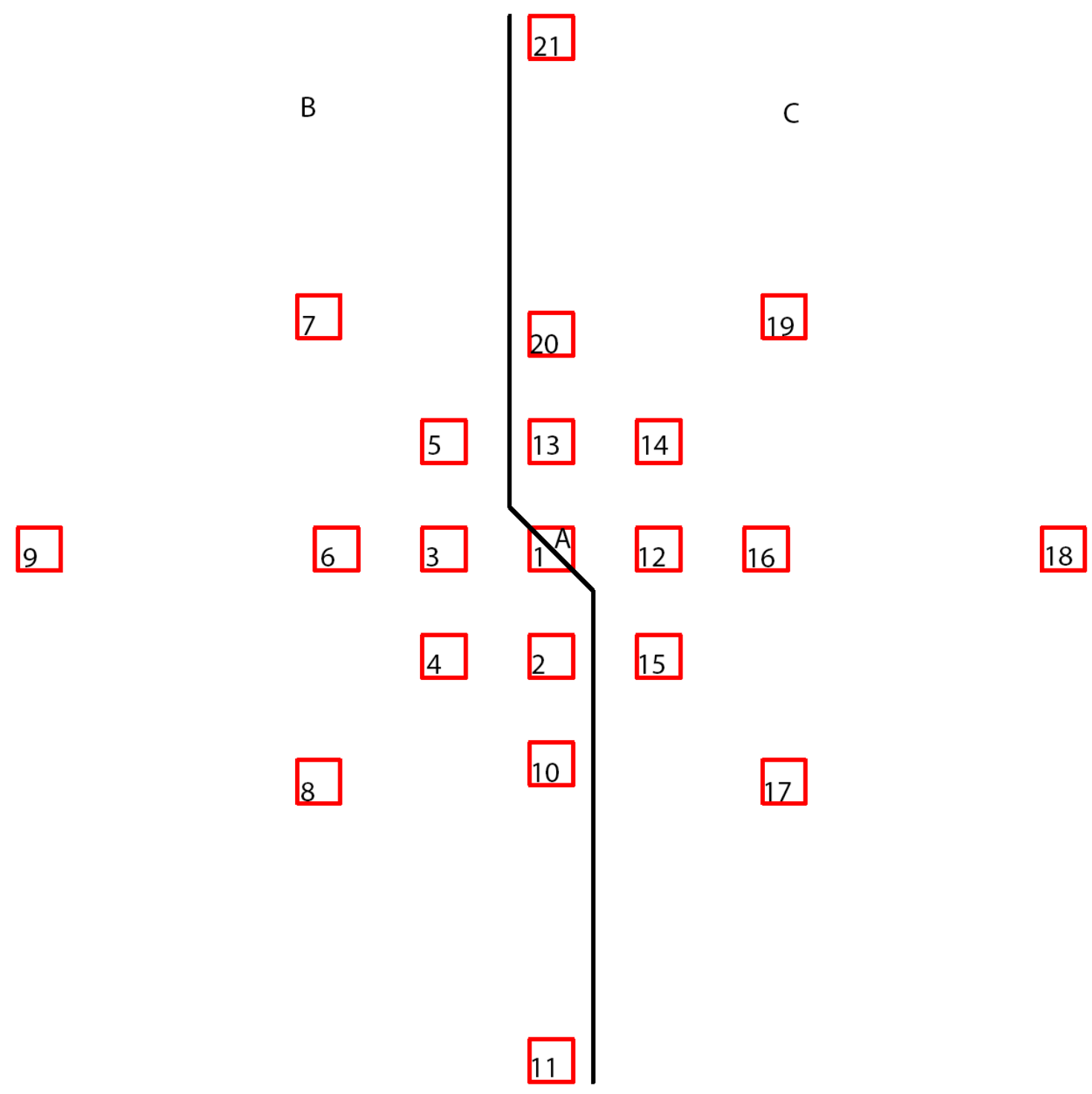

Figure 4.5: Symmetry in patch layout.

Besides the symmetry, the major advantages to dividing the feed network is that all three sections require a weighting of approximately 1, with the two other sections requiring $10 \%$ more power. The table of weightings is shown below with the numbers corresponding to patches in Table 4.1. 
Table 4.1: Weighting of patches.

\begin{tabular}{cccc}
\hline Patch Number & Section & Power Weighting & Sum of Powers \\
\hline \hline 1 & A & 1 & 1 \\
\hline 2,3 & B & 0.36 & \\
4,5 & B & 0.16 & 1.1 \\
$6,7,8,9,10,11$ & B & 0.01 & \\
12,13 & C & 0.36 & 1.1 \\
14,15 & C & 0.16 & \\
$16,17,18,19,20,21$ & C & 0.01 & \\
\hline
\end{tabular}

Since this creates an even number of patches with each weighting, in either section, half the patches could be on one branch of the feed network and half on the other. A second option could have been to use a three way split that fed each different weighting. This alternative method would not have had any symmetry and would have required a splitter that divided the power in a way that would be impossible to manufacture. Since each branch had an even number of patches with each different weighting, the feed could be considered in quarters ensuring each quarter receives the same phase and power.

The length of the line determines the electrical length or phase being delivered to each patch. The type and thickness of the substrate also plays a role in determining the electrical length through the following equation:

$$
\phi=\beta \mathcal{L}=\sqrt{\epsilon_{e}} k_{0} \mathcal{L}
$$

Where $\epsilon_{e}$ is:

$$
\epsilon_{e}=\frac{\epsilon_{r}+1}{2}+\frac{\epsilon_{r}-1}{2} \frac{1}{\sqrt{1+12 d / W}}
$$


The dielectric constant is a property of the material, but the effective dielectric constant shown above in equation 4.2 illustrates that the thickness of the material and the width of the microstrip line also influence the electrical length of the line. In order to ensure correct phases, the lines on the feed network were measured using Autocad and then were input to the ADS circuit in Figure 4.6 to approximate the phases at each port. 


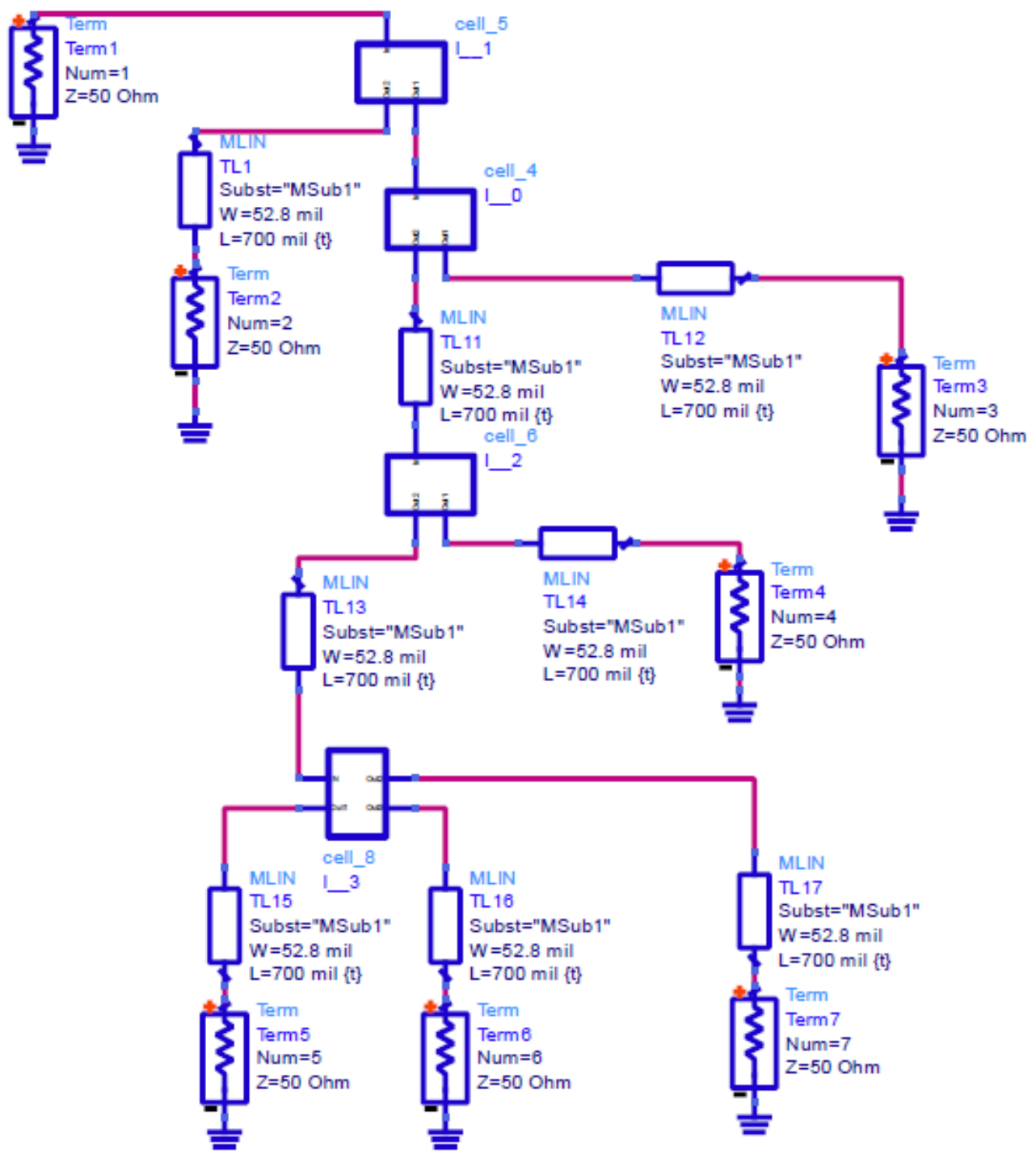

Figure 4.6: Section of the feed network designed in ADS.

This procedure provided an estimate of what phases could be expected. However, given that the ADS model does not account for the corners and bends in the layout, the phases are not exact. The other cells in the diagram are power dividers that will be discussed in Section 4.4. The feed network was developed in branches: once one branch was complete, the other was designed to be identical in length (although the 
layout would be slightly different due to lack of symmetry). After the network was designed in ADS, the feed was simulated in Empire to determine if the powers and phases delivered to each port were correct. As noted above, given the bends were not modeled in ADS it required, some adjustments in order to match phases at all ports.

Figure 4.7 shows the full layout of the feed network in Empire, with the exception that the outer patches will be fed with a cable because of limitations on the board size from the manufacturer. To ensure symmetry, half the feed network was designed as mentioned above, and the other half was a rotated 180 degree copy. The patches on the right half are fed in their opposite port compared to those on the left half. In order to account for the phase difference going into the patches, a 180 degree electrical length line was placed after the first three way divider. The U shaped patterns used throughout are used to adjust the phase to certain sections of the network.

During the design process amplitudes and phases would be tested by placing ports at the ends where patches would go. In an iterative process the phases and amplitudes of the feed network were determined. The outer patches however, were fed by cables. The electrical lengths of the cables were determined by measuring the phase of the open ends (that would eventually connect to the outer patches), to ensure the phase is 180 degrees off the main network. 


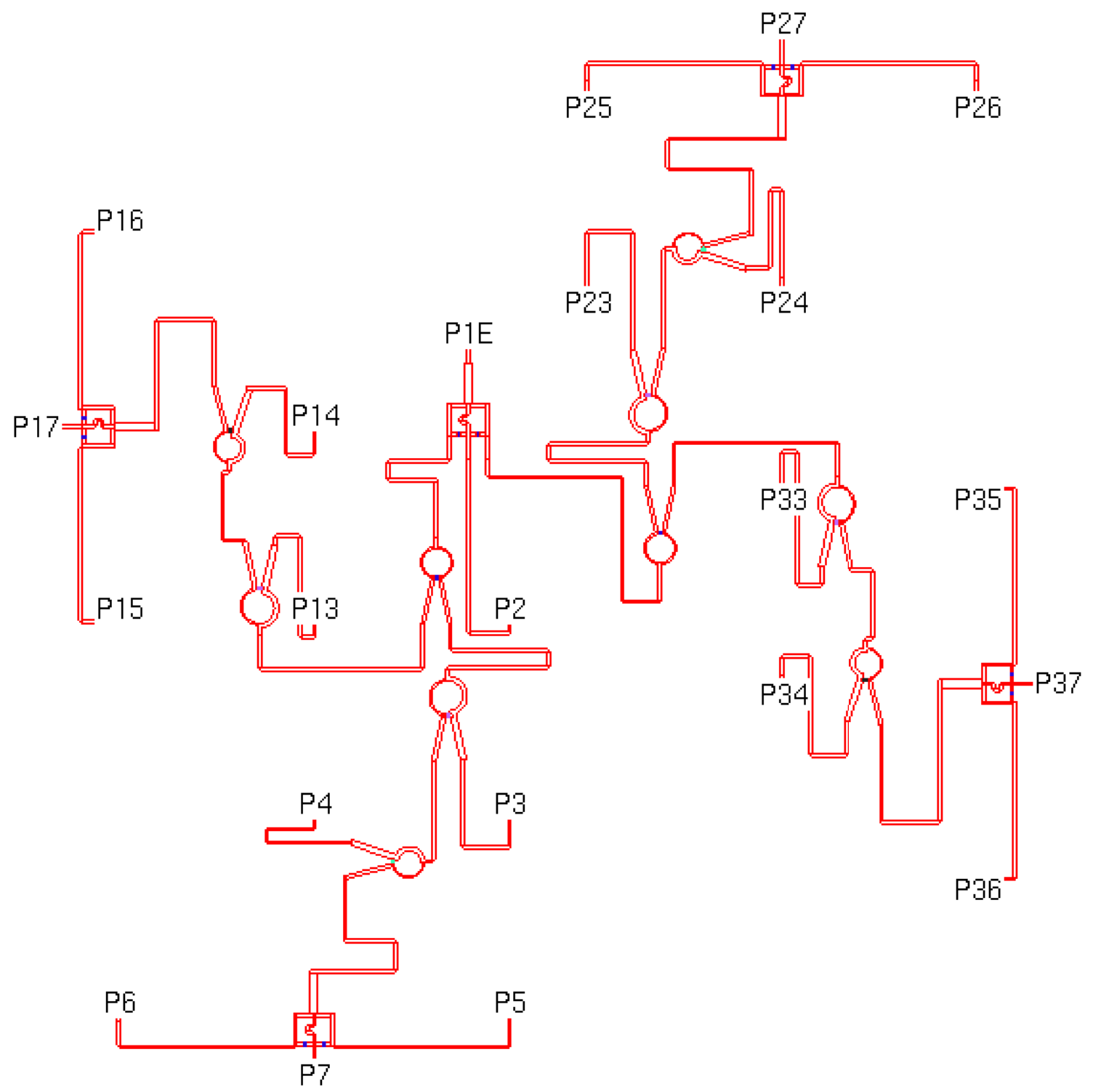

Figure 4.7: Full layout of feed network.

\subsection{Power Dividers}

The power dividers primary role is to split the power correctly to meet the power requirements as determined in Chapter 3 and Table 4.2 above. The power required at each split was determined by a bottom up approach: an equal 3-way split was 
required at the very bottom. The total sum of power at the input needed to be 0.03 . At the next junction, a patch was a power of 0.16 needed to be split with 0.03 , however this divider would require a microstrip line that was too thin, therefore the power going to the three way divider was doubled to 0.06 . This required a $-3.52 \mathrm{~dB}$ power divider. Further up, the power needed to be split between 0.22 and 0.36 , required a $-2.15 \mathrm{~dB}$ splitter. The final splitter is an equal power split providing power to both branches with the same values as above. This requires a $-3 \mathrm{~dB}$ power splitter.

The four different splits are outlined in Table 4.2 with the required impedances to achieve the splits in a Wilkinson Divider.

Table 4.2: Power splitter impedances.

\begin{tabular}{cccccc}
\hline Divider & Split & Upper Arm 1 & Upper Arm 2 & Lower Arm 1 & Lower Arm 2 \\
& $(\mathrm{dB})$ & $(\Omega)$ & $(\Omega)$ & $(\Omega)$ & $(\Omega)$ \\
\hline \hline A & -3 & 70.7 & 70.7 & 50 & 50 \\
B & -3.52 & 42 & 94.5 & 34.9 & 52.4 \\
C & -2.15 & 42 & 68.9 & 33.1 & 42.4 \\
D (Three Way) & -4.78 & 28.8 & 50 & 50 & 50 \\
\hline
\end{tabular}

The Wilkinson power dividers were used to provide isolation between all ports - an advantage over T-Junction splitters. The ring design was selected because it allowed for the ends to be connected via a surface mount resistor instead of a through-hole resistor. This configuration reduces the potential for coupling because the microstrip lines are further spaced then if they were straight. The lengths and widths were determined using the LineCalc tool in ADS. The four different dividers are shown in Figure 4.8 below. 


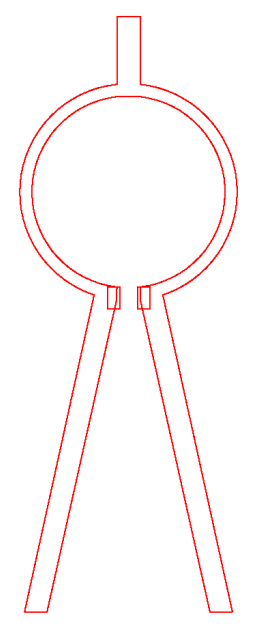

Divider A

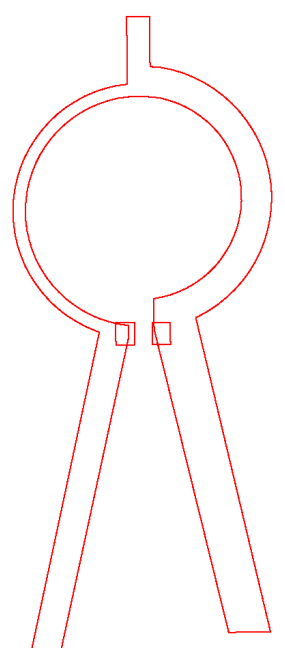

Divider B

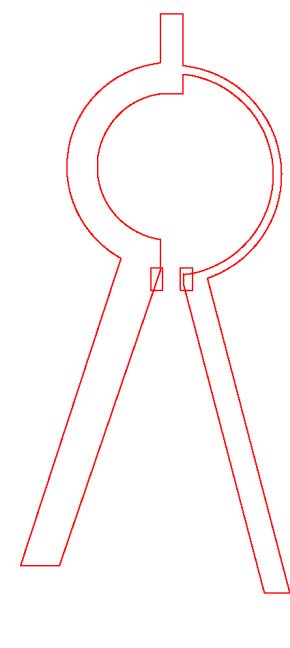

Divider C

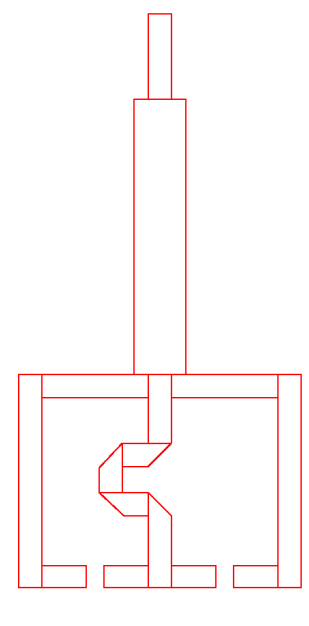

Divider D

Figure 4.8: Layout of power dividers.

The first divider is the equal split: both upper arms are the same widths with the lower arms being $50 \mathrm{ohms}$. The second and third dividers are the unequal dividers. The dimensions of these dividers are in Tables 4.3 - 4.6.

Table 4.3: Divider A Dimensions.

\begin{tabular}{ccc}
\hline & Width (mil) & Length (mil) \\
\hline \hline Left Upper Arm & 28 & 723 \\
Right Upper Arm & 28 & 723 \\
Left Lower Arm & 52 & 699.4 \\
Right Lower Arm & 52 & 699.4 \\
\hline
\end{tabular}


Table 4.4: Divider B Dimensions.

\begin{tabular}{ccc}
\hline & Width (mil) & Length (mil) \\
\hline \hline Left Upper Arm & 69.4 & 715 \\
Right Upper Arm & 29.9 & 740.7 \\
Left Lower Arm & 97.8 & 704.1 \\
Right Lower Arm & 68.4 & 715.5 \\
\hline
\end{tabular}

Table 4.5: Divider C Dimensions.

\begin{tabular}{ccc}
\hline & Width (mil) & Length (mil) \\
\hline \hline Left Upper Arm & 69.4 & 715 \\
Right Upper Arm & 19.6 & 751.7 \\
Left Lower Arm & 93 & 705.7 \\
Right Lower Arm & 55.5 & 722.1 \\
\hline
\end{tabular}

Table 4.6: Divider D Dimensions.

\begin{tabular}{ccc}
\hline & Width (mil) & Total Length (mil) \\
\hline \hline Upper Arm & 118 & 698 \\
Left Lower Arm & 52 & 699.4 \\
Center Lower Arm & 52 & 699.4 \\
Right Lower Arm & 52 & 699.4 \\
\hline
\end{tabular}

The three way divider design resemble Wilkinson power dividers; although, because the two outside arms of the divider are not connected with a resistor they are not Wilkinson power dividers. This divider was designed using ADS with the layout in Figure 4.9. The impedance of the first section of the divider was determined using:

$$
Z=\frac{Z o}{\sqrt{N}}
$$


Where $\mathrm{N}$ is the number of splits with the remaining lines being 50 ohms. A sweep of resistor values identified the optimal value for both isolation and costs. The results are shown in Figure 4.10: with the resistor the same as Divider A (100 ohm), the number of different components is reduced.

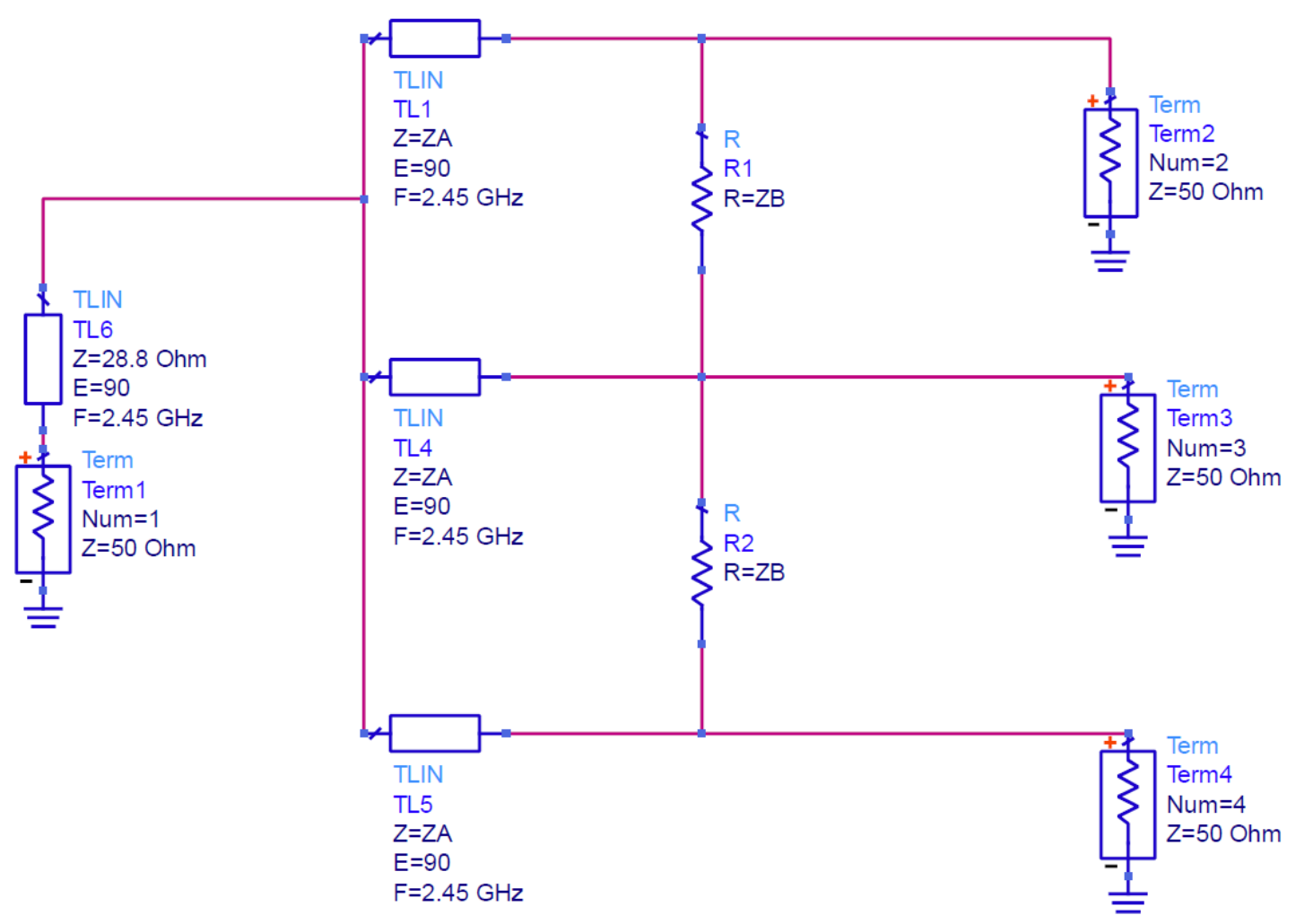

Figure 4.9: ADS Layout for Modified Wilkinson. 


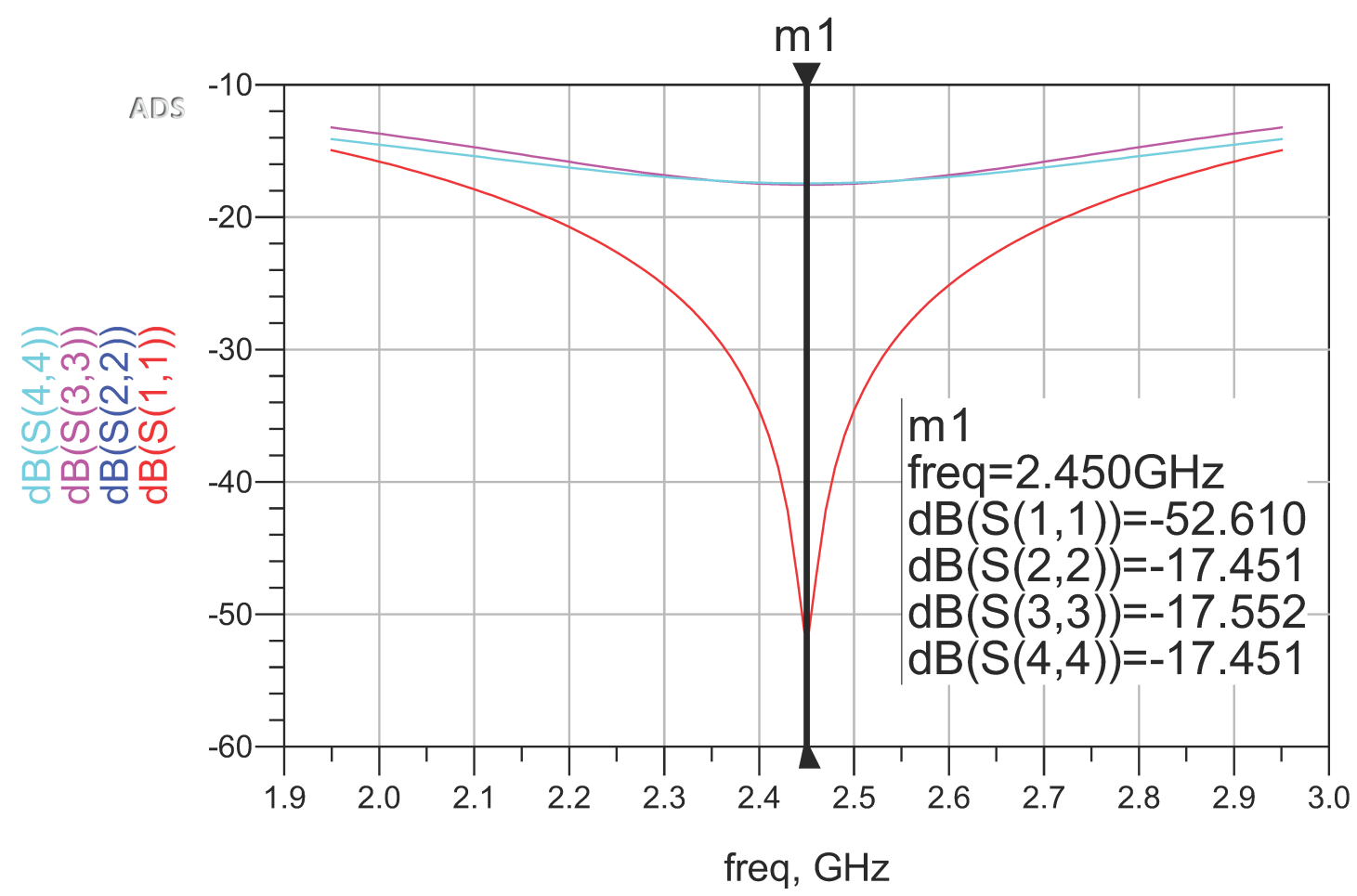

Figure 4.10: Reflection Coefficient of Modified Wilkinson.

These results demonstrate an excellent match at the input of the divider with a return loss of $-52.6 \mathrm{~dB}$. As less than $2 \%$ of the power is reflected back, it also provides a balanced match at all the output ports. The simulated results from Empire for all dividers are shown in Table 4.7.

Table 4.7: Simulated scattering parameters of dividers.

\begin{tabular}{ccccc}
\hline Divider & S11 & S12 & S13 & S14 \\
\hline \hline Divider A & $-18.6 \angle 44.3$ & $-3.0 \angle 124.8$ & $-3.06 \angle 124.9$ & \\
Divider B & $-25.0 \angle-159.1$ & $-2.04 \angle 111.8$ & $-4.29 \angle 113.1$ & \\
Divider C & $-16.32 \angle 55.6$ & $-4.66 \angle 133.3$ & $-1.99 \angle 141.5$ & \\
Divider D & $-23.2 \angle-60.1$ & $-4.86 \angle 157.2$ & $-4.8 \angle 157.2$ & $-4.86 \angle 156.4$ \\
\hline
\end{tabular}




\subsubsection{Switch to modified Wilkinson}

During the design process a T-junction three way splitter was originally used for all the three way splitters. This produced the radiation pattern illustrated in Figure 4.11. These poor results with the T-junction is likely attributed to the lack of isolation between ports and the feed networks reflections.

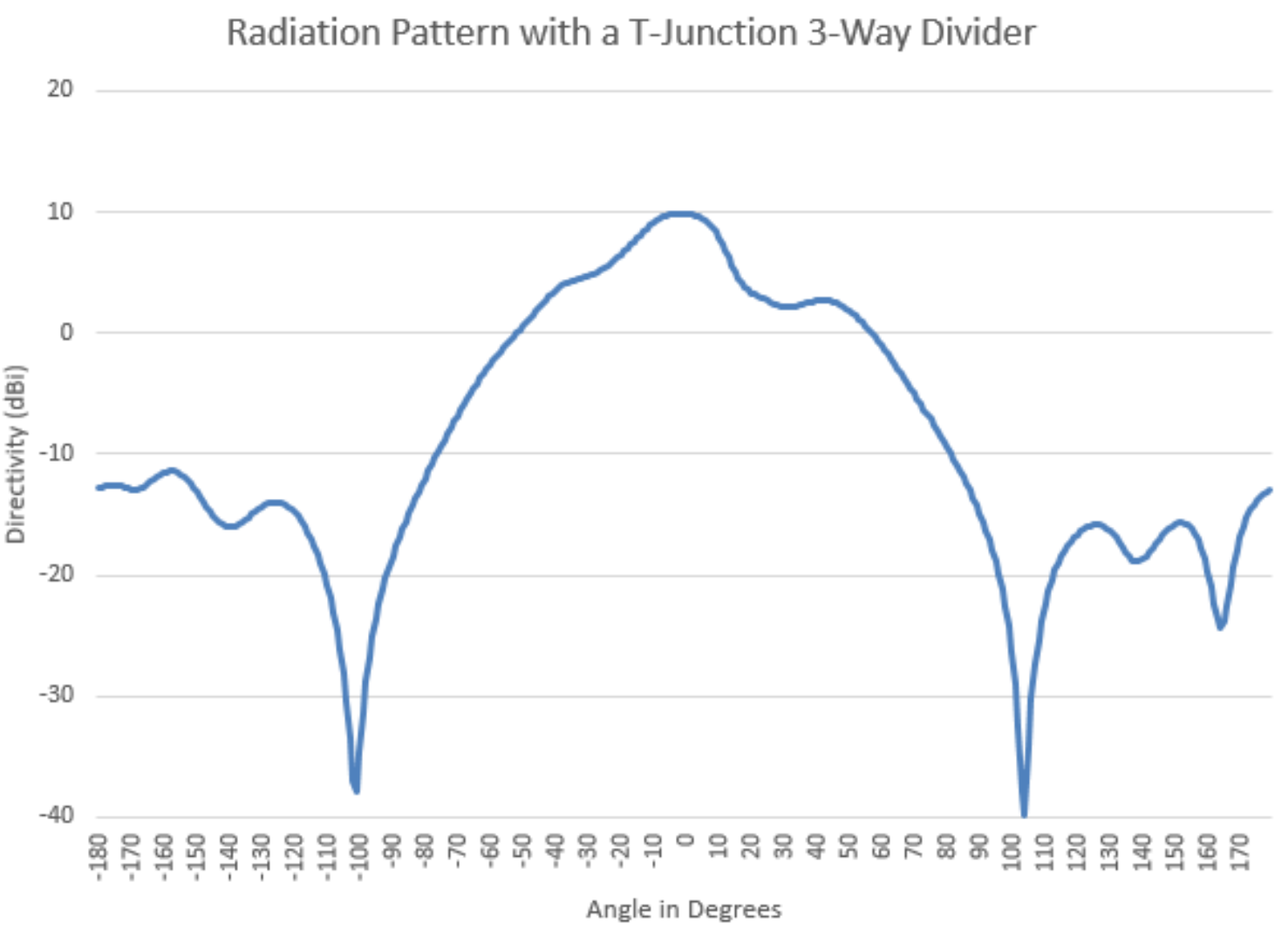

Figure 4.11: Radiation pattern with a T-Junction Divider.

Substituting the T-junction with the modified Wilkinson, produced more desirable results as shown in Figure 4.12. It is modified given there are only two resisters as opposed to having the third bridging the two outer arms. This results in all 3 ports not being perfectly matched [21], however the results in Figure 4.12 indicate that this is negligible. 


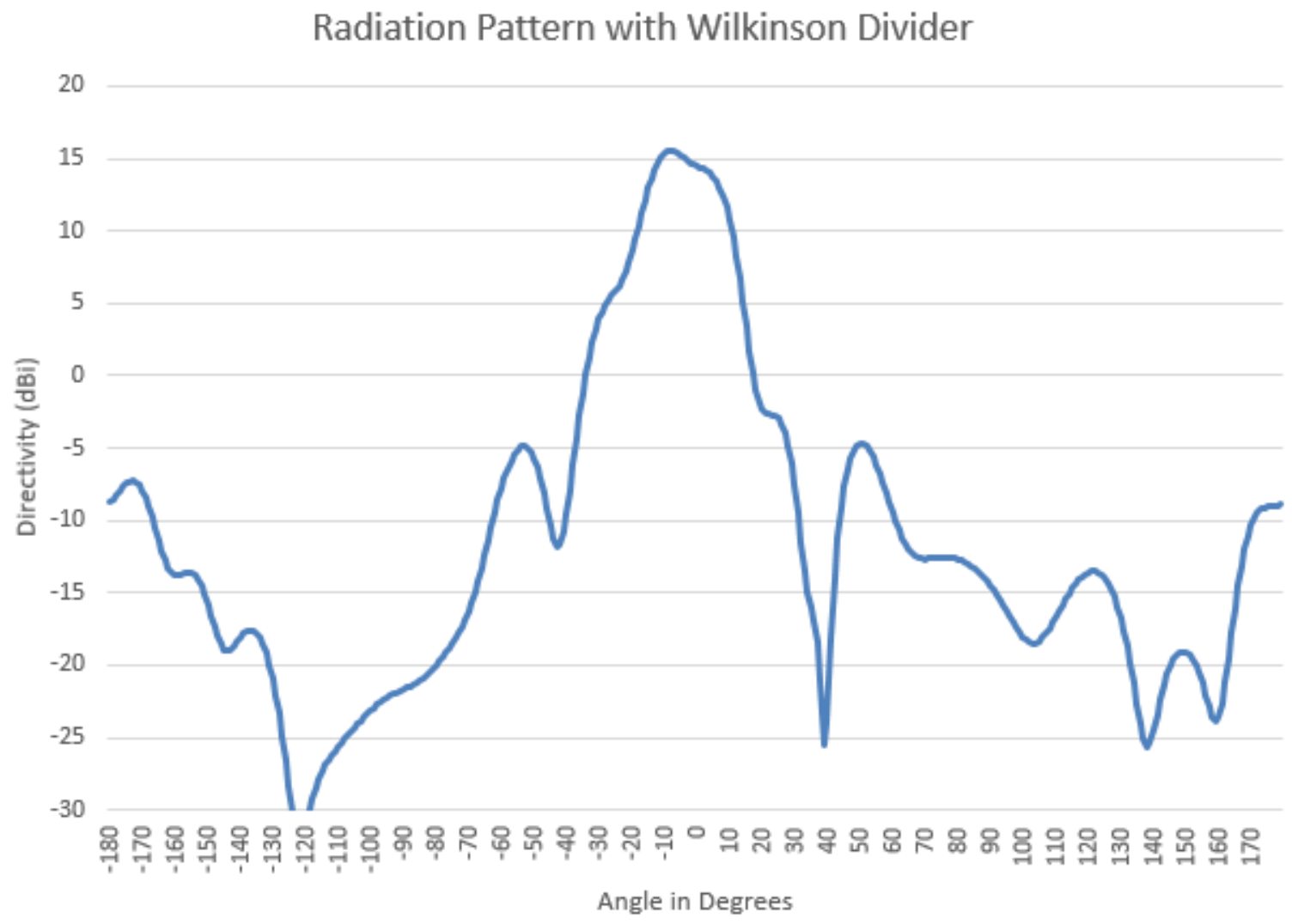

Figure 4.12: Radiation pattern with a Modified Wilkinson Divider.

The pattern in Figure 4.12 was formed by the same array as above with the exception that the first divider is now the modified Wilkinson. While this pattern is un-optimized compared to that of Section 4.7 it is a vast improvement over the T-Junction. In this layout, the outside dividers are still basic T-Junctions, however they were later replaced by the same three way modified Wilkinson dividers in order to reduce the number of different circuit blocks.

\subsection{Tuning Stubs}

Patches were simulated with ideal phases and weighting also taking into account the effects of mutual coupling [22]. This resulted in a range of return losses. On average, 
the return loss at each patch was $-3 \mathrm{~dB}$. The mismatch from the patches caused the return loss of the whole system to be effected.

Tuning stubs at each of the main patches are required because the mutual coupling between the patches in the array caused non-ideal impedances and produced a system return loss of $-4.9 \mathrm{~dB}$.

Impedance matching techniques were calculated using a Smith chart [23]. It was determined that a stub size of 1049 thou at a distance of 132 thou from the patch should be used. This stub was used on the inner nine patches. It was not necessary to stub match the outer patches as the power delivered was minimal and therefore, the reflections would not cause a significant problem. The improvement to individual patches with the use of stub matching is shown in Figure 4.13.

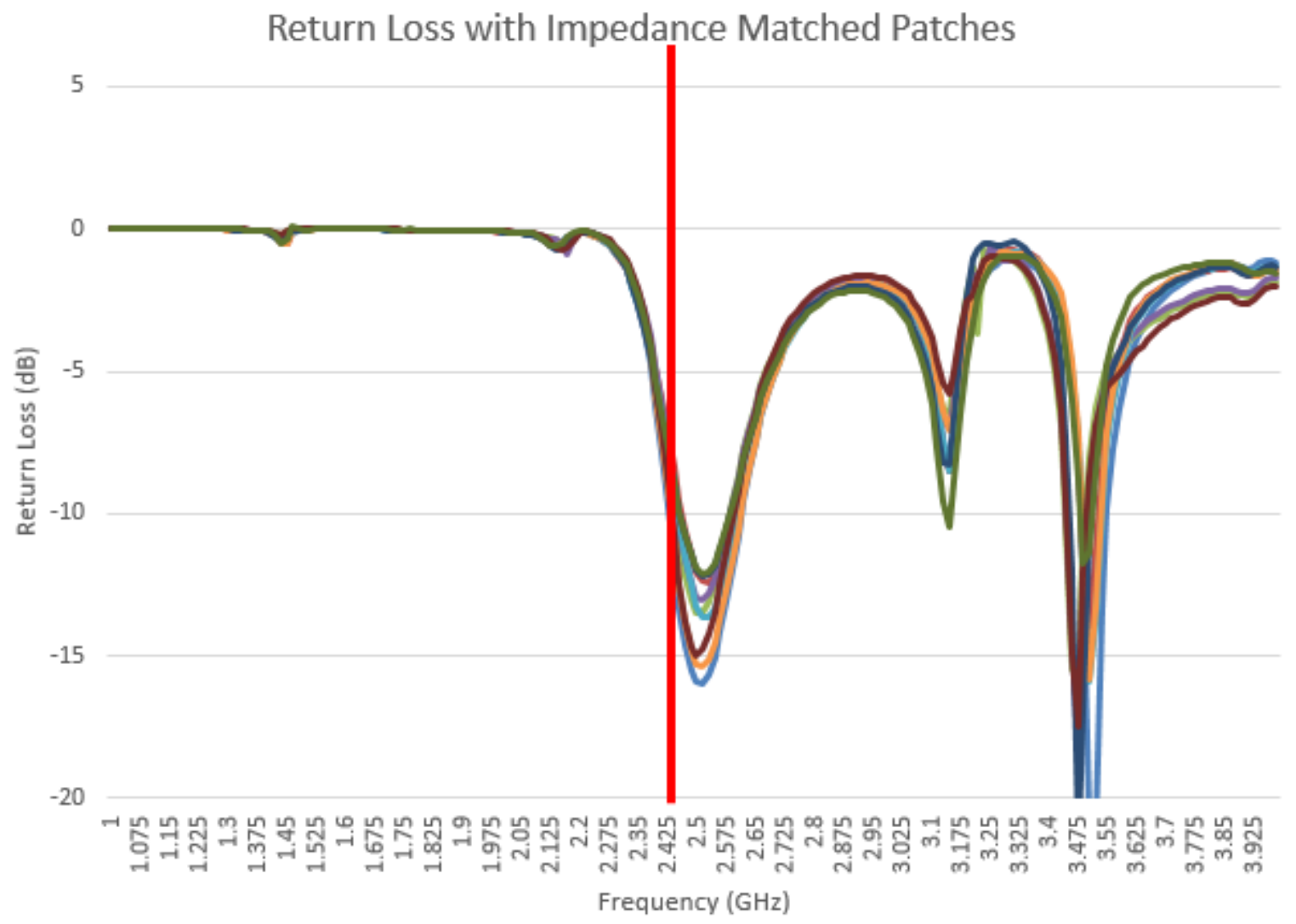

Figure 4.13: Return loss of the patches with tuning stubs. 
The performance improved from an average of $-3 \mathrm{~dB}$ to between $-7.93 \mathrm{~dB}$ and $10.97 \mathrm{~dB}$ with the use of the stubs. Even with the tuning stubs, the results of the whole feed network are still undesirable: as a total system, it still produced a return loss of $-8.66 \mathrm{~dB}$.

\subsection{Return Loss Methodology}

The return loss levels were still below the goals because the reflections in a feed network add coherently. Any reflection from a mismatch adds throughout the network.

The three way modified Wilkinson is at the beginning of the feed network. The left and right sections at the exact same except the right arm is rotated 180 degrees. The center arm of the divider feeds the middle patch. The two outer arms cancel each other because of the 180 degree phase shift on the right side so the remaining reflection will be from the center patch. This works if all reflections are perfectly matched; however not all the reflections would be cancelled. The resultant is only decreased with respect to the overall reflection. The center patch can be used to cancel out the resultant reflection. The center reflection needed to be larger than the resultant, so the center patch was left mismatched, while the remaining eight patches (matched in Section 4.5) were left the same.

In order to cancel the reflections, lengths of line needed to be added to three way divider in order to facilitate the cancelation (otherwise the phase might not have been correct to cancel the reflections). The limiting factor was the length of time to run a simulation (they took over a day to run). In order to determine the optimal length changes, many simulations were required. To reduce simulation time, the left side of the array was fed with the feed network, while the right side was fed with ideal patches so the impedance for each side could be determined. These results were then 
put into a simplified simulation that only included the three way divider (shown in Figure 4.14) where the lengths of each arm are varied to determine their optimal position.

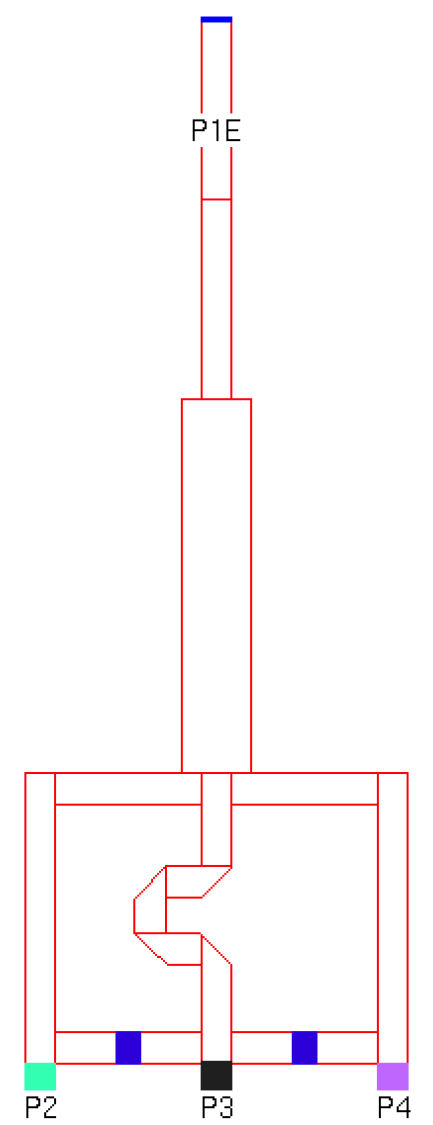

Figure 4.14: Schematic used to determine optimal length of divider arms.

After a series of simulations, results showed that adding 360 thou of line to each feed allowed the phases to be matched in such a way that the return loss dropped from $-12.68 \mathrm{~dB}$ in the initial simulation to $-25.83 \mathrm{~dB}$ with the added length. The 360 thou line was added to the full feed network with patches and another simulation resulted in a return loss of $-11.85 \mathrm{~dB}$ at $2.45 \mathrm{GHz}$ with a $-10 \mathrm{~dB}$ bandwidth of $2.38-2.67 \mathrm{GHz}$. 


\subsection{Radiation Pattern}

The radiation pattern was examined after the return loss reductions were completed. The full simulation achieved the results illustrated in Figure 4.15. The sidelobe levels in Figure 4.15 are much higher than the sidelobes in Chapter 3's simulations.

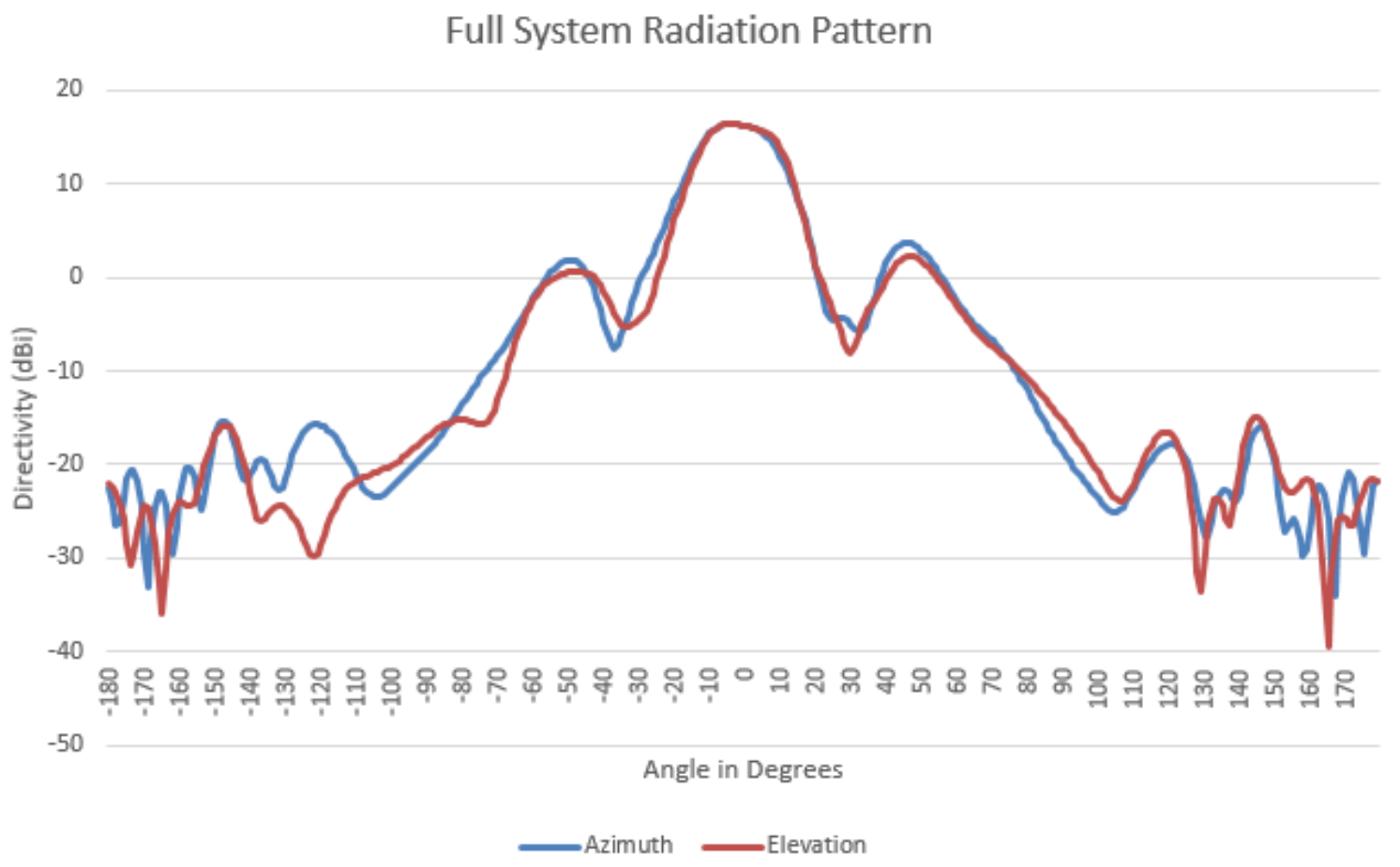

Figure 4.15: Radiation pattern of the full system in Azimuth and Elevation.

To investigate the phases and magnitudes being delivered to each patch, probes were inserted before every patch and other key locations as shown in Figure 4.16. This simulation provides insight to the E-Fields phase in the feed network to see how different the expected results from Chapter 3 and Section 4.3 are when introduced to reflections. This information is shown in Table 4.8 alongside the ideal network and the feed network's magnitudes and phases. The angles are normalized to the values obtained for the center patch (-7.5 for the feed network and 149.8 for the full system). Ideal in this section refers to if everything was matched and split perfectly without 
any losses.

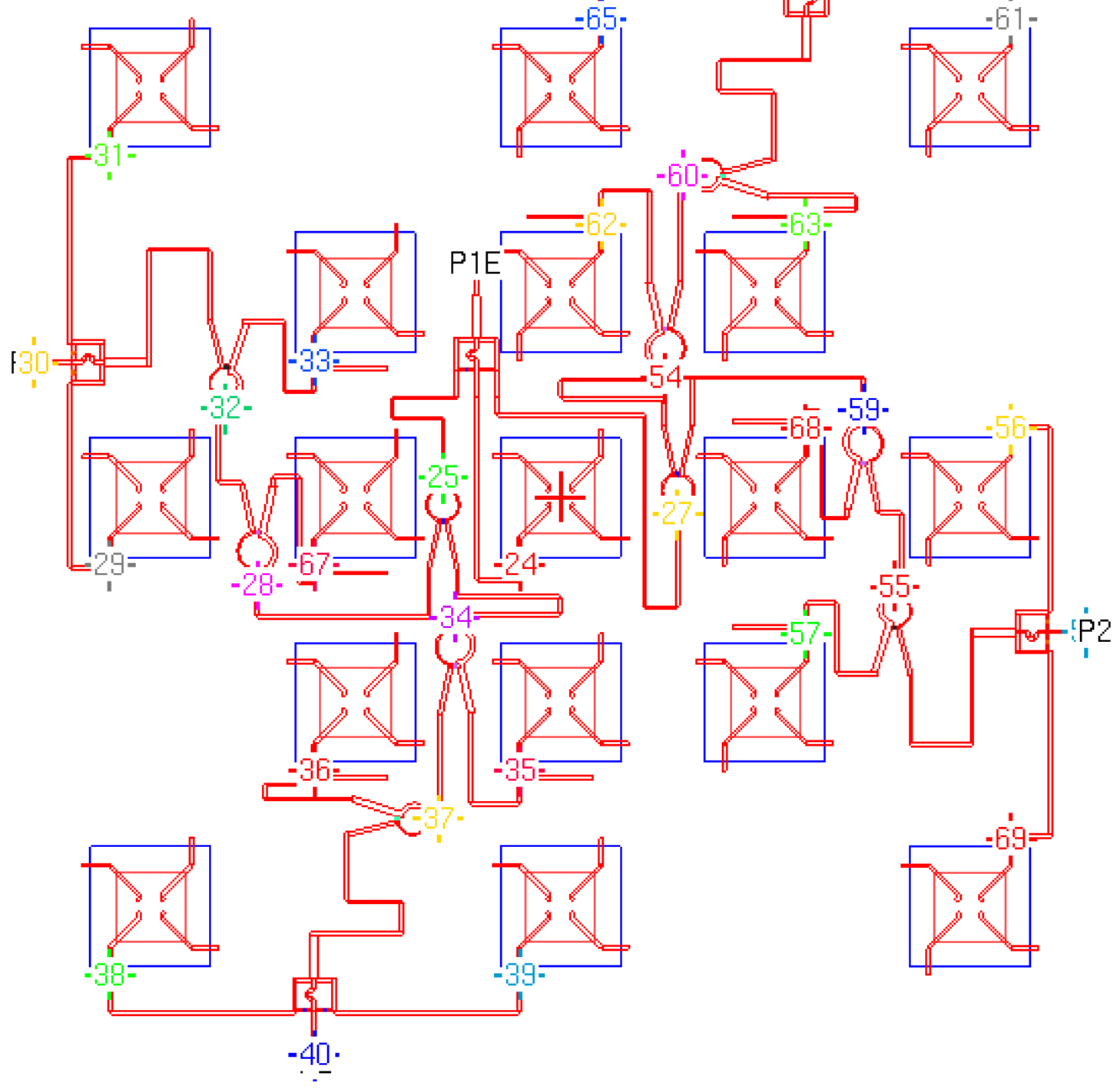

Figure 4.16: Layout of port field probes. 
Table 4.8: Comparison between ideal, feed network alone, and full system.

\begin{tabular}{cccc}
\hline Patch Number & Ideal & Feed Network & Full System (phase) \\
\hline \hline 2 & $0.577 \angle 0$ & $.597 \angle 0$ & 0 \\
3 & $0.319 \angle 0$ & $0.303 \angle-9.3$ & 44.4 \\
4 & $0.191 \angle 0$ & $0.185 \angle-10$ & 48.6 \\
5 & $0.098 \angle 0$ & $0.073 \angle-9.3$ & 7.8 \\
6 & $0.098 \angle 0$ & $0.073 \angle-10.8$ & 5.2 \\
7 & $0.098 \angle-155$ & $0.074 \angle-38$ & -4.5 \\
13 & $0.319 \angle 0$ & $0.307 \angle-2.6$ & 41.9 \\
14 & $0.191 \angle 0$ & $0.189 \angle-5.6$ & 46.2 \\
15 & $0.098 \angle 0$ & $0.074 \angle-2.6$ & 2 \\
16 & $0.098 \angle 0$ & $0.072 \angle-8.8$ & -2.2 \\
17 & $0.098 \angle-155$ & $0.072 \angle-21.8$ & -0.4 \\
23 & $0.319 \angle 180$ & $0.314 \angle 68.8$ & -128.9 \\
24 & $0.191 \angle 180$ & $0.195 \angle 65.6$ & -118.6 \\
25 & $0.098 \angle 180$ & $0.077 \angle 63.7$ & -159.8 \\
26 & $0.098 \angle 180$ & $0.078 \angle 67$ & -166.4 \\
27 & $0.098 \angle 25$ & $0.078 \angle 157.1$ & -177.2 \\
33 & $0.319 \angle 180$ & $0.314 \angle-162.9$ & -127.9 \\
34 & $0.191 \angle 180$ & $0.195 \angle 73$ & -123.8 \\
35 & $0.098 \angle 180$ & $0.075 \angle 73.5$ & -176 \\
36 & $0.098 \angle 180$ & $0.077 \angle 68.3$ & -177.4 \\
37 & $0.098 \angle 25$ & $0.075 \angle 55.3$ & -166.7 \\
\hline & & &
\end{tabular}

This demonstrates a difference in magnitude and phase between the ideal and the feed network. The full system has a number of patches' phase that are different from the feed network. In particular the phase to the center group of patches are about 45 degrees off of what is expected. One explanation is that the reflections from the mutual coupling causes the phase shifts as the reflected wave contribute to the overall 
phase. This makes it difficult to predict how the phase will react to changes in lengths of lines.

Using the layout from Chapter 3 an investigation into the phases that effected the pattern the most was undertaken. The test plan started with the highest powered patches and adjustments to the phases were made one at a time to observe the effects. This study illustrated that the patches receiving an amplitude of $0.4 \mathrm{~A}$ were the largest contributor to the pattern. A simulation was run to test the phases with the lines going to these patches adjusting up and down 100 thou. These results to the radiation pattern are shown in Figure 4.17. It produced a return loss of $-11.3 \mathrm{~dB}$ at $2.45 \mathrm{GHz}$ and a $-10 \mathrm{~dB}$ bandwidth of $2.38 \mathrm{GHz}$ to $2.466 \mathrm{GHz}$.

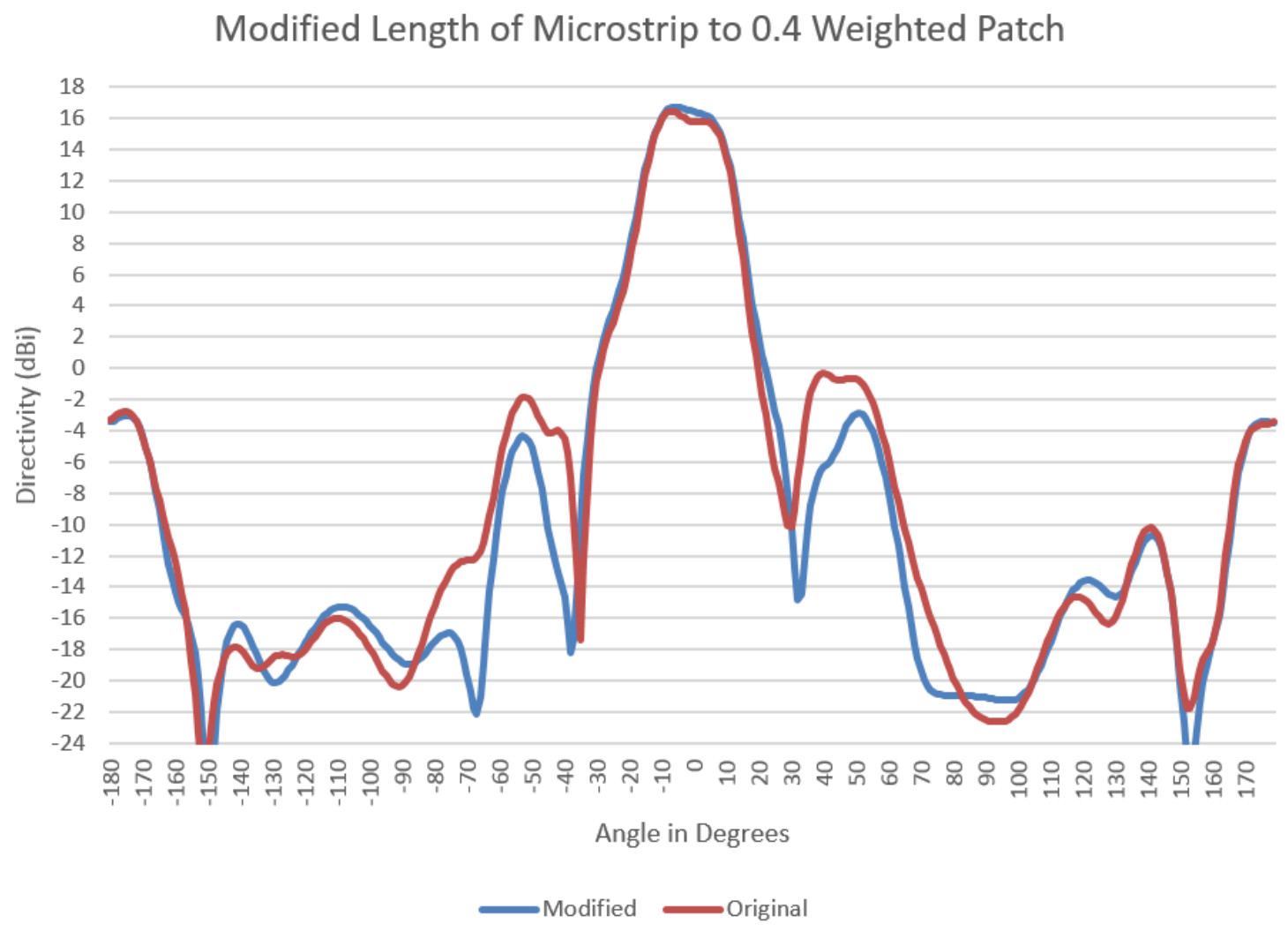

Figure 4.17: Modified microstrip line lengths effects on radiation pattern.

This change in length caused a change in reflections at the three way divider so 
the process in Section 4.6 needed to repeated. Given the improvements in return loss were still lower than the results in Section 4.6, it was decided that having good return loss across was more important than slightly lower sidelobes.

\subsection{Conclusions}

The final simulations of the complete network and patch array in Figure 4.15 show that the results have degraded with sidelobe levels of $12.8 \mathrm{~dB}$.

Since the results of the full simulation with the feed network were not as expected, an investigation was completed to determine the values of the phase and power at each port with the patches still in place. The values obtained were used to directly feed the patches in the array in order to determine if the feed network was the cause of poor performance. The results of this test can be seen in Figure 4.18. 


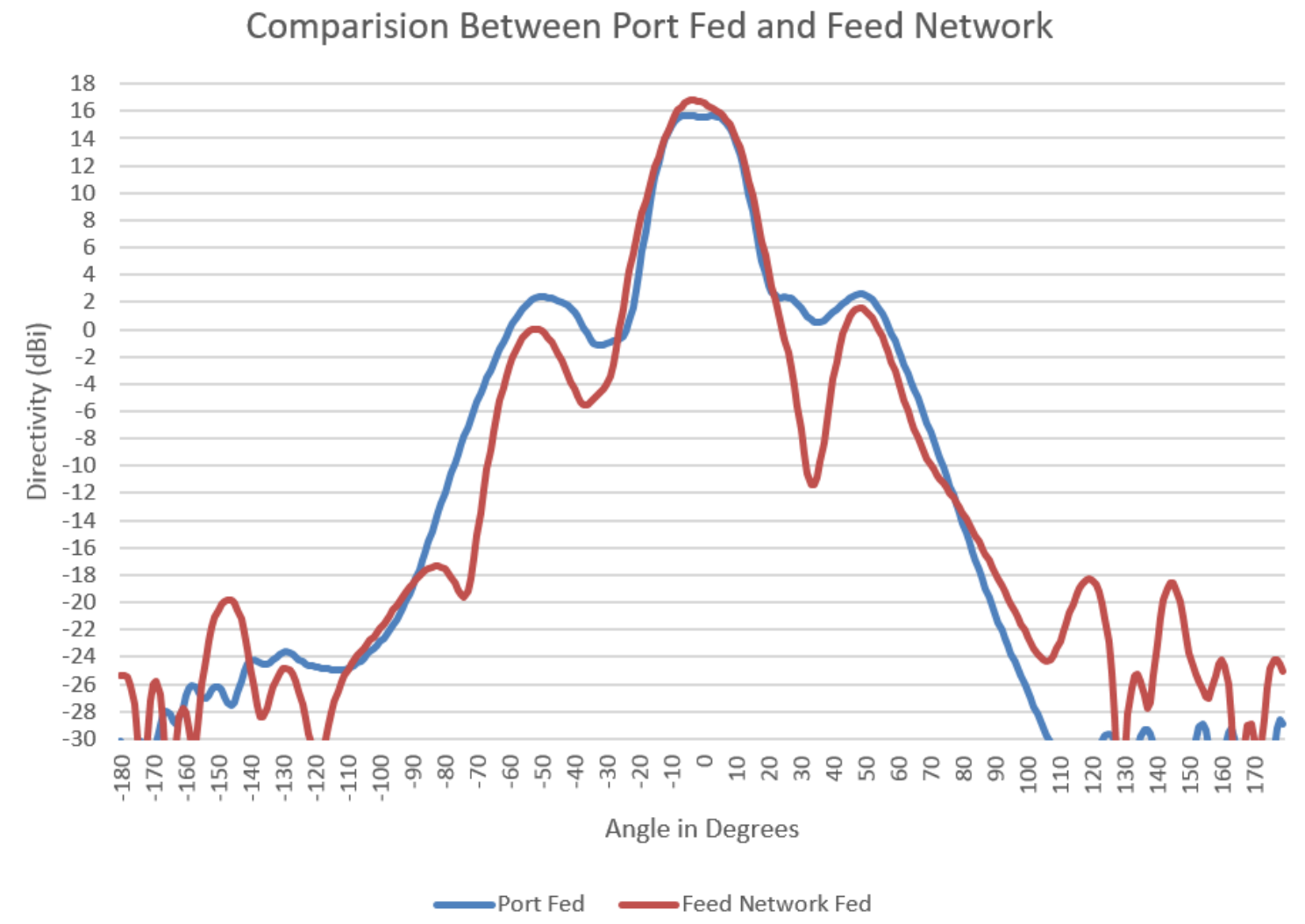

Figure 4.18: Comparison between port fed array and feed network array

As demonstrated in Figure 4.18, when the port fed array is fed with the values obtained from the feed network, the results are very similar, with a sidelobe difference of just $2 \mathrm{~dB}$. In conclusion, if the feed network was optimized to provide the correct power and phase to each patch the results would be similar to that of Figure 3.26. 


\section{Chapter 5}

\section{Testing Results}

\subsection{Introduction}

The antenna design was migrated to Autocad to be able to produce drawings required by VIA systems to manufacture the boards. There were three panels laid out: the feed and sub-array in RO4003, the patches in FR4, and a board with break outs for testing of individual components, in RO4003. The entire system was tested to verify that a square beam will be produced. The breakouts were to verify sections of the feed network matched the simulation results, and show required alterations to the components in enhancing the system level results. The five parameters tested in the overall system consist of: Gain, Sidelobe level, Radiation Pattern (Squareness), return loss, and beamwidth.

\subsection{Fabrication}

As previously mentioned, fabrication was carried out by VIA Systems in Toronto, Canada. The remaining components were purchased from various suppliers. Resistors for the power dividers were soldered on to the board at Carleton University and 
spacers were used to provide the necessary air gap between the RO4003 and FR4.

Cables were purchased from Superlink to provide approximately -170 degrees of phase difference. The cables were necessary because the original board was too large for VIA to manufacture. Spacers were designed to anchor the outer patches in the correct location and the cables connect the feed out to the inverting patches. The cables were RG-316, despite not having the best phase performance, due to cost and the outer patches not being sensitive to phase differences. In sensitivity studies, phase to outer patches did not show sensitivity up to 50 degrees (Figure 5.1). The major effect is the loss of squareness as the phase offset from 180 degrees becomes too large. Two sets of cable lengths were manufactured to ensure they were long enough to reach the outer patch but not be excessively long. One set of cables were 9.11 inches while the other was 12.41 inches long. Both sets were designed to have phases of approximately -170 degrees. 


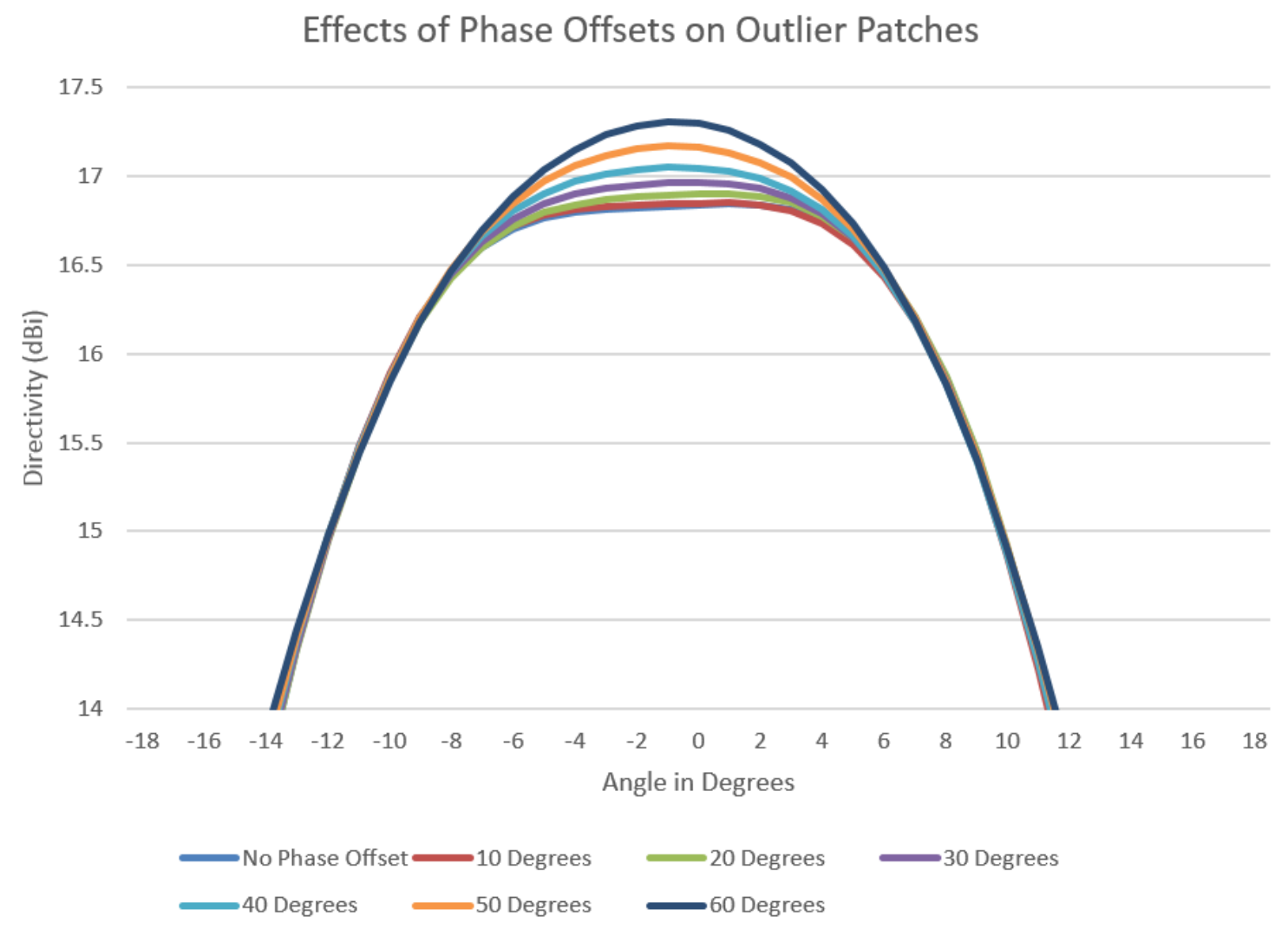

Figure 5.1: Effects on squareness with different phase offsets.

The phase length of the cable can be determined by the velocity factor of the cable, as well as the tolerance by the manufacture. The tolerance on length was $+/-$ $3.5 \mathrm{~mm}$ on the cable, which meant a phase tolerance of $+/-14.8$. This is well within the limits found in the sensitivity study.

Two sets of spacers were used to see the effect of the air gap on S11 and bandwidth. The results are shown in Section 5.4. Nylon screws were used to secure the spacers since they are non-conductive and will not interfere with the radiation pattern.

The final array can be seen in Figure 5.2 and the full feed network in Figure 5.3. The array was mounted to an aluminum ground plane to maintain rigidity while testing. 


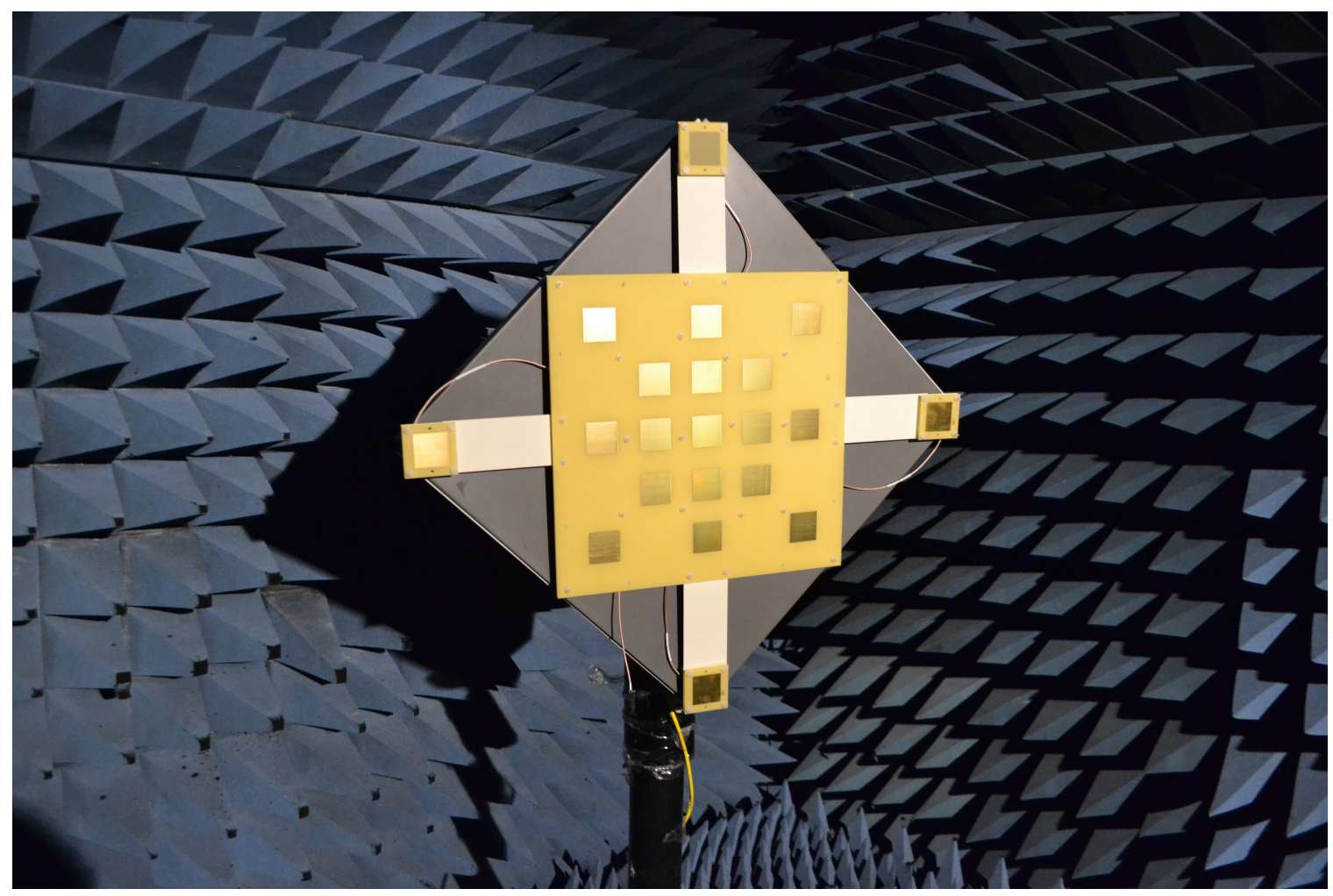

Figure 5.2: Photo of array inside the anechoic chamber.

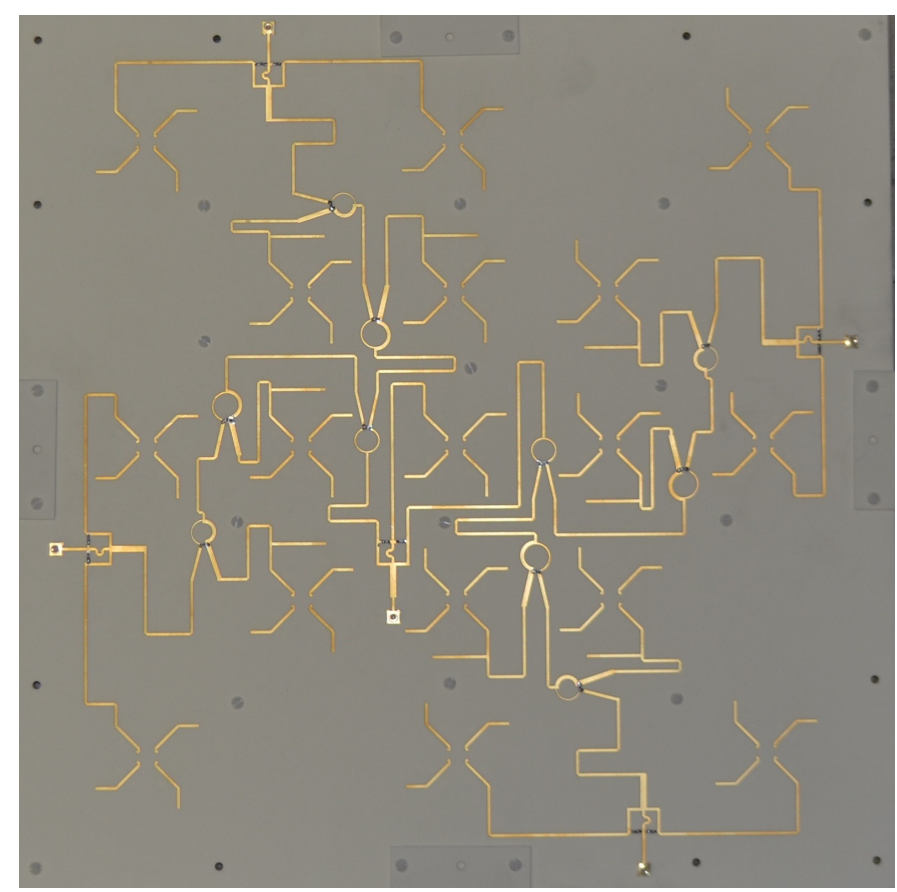

Figure 5.3: Photo of the feed network. 
A variety of breakouts were also manufactured in order to test the performance of each individual section of the feed network. Each unique card was tested and the results of each divider and section of feed are explored in Section 5.4.

\subsection{Test Methodology}

The scattering parameters were measured using a HP 8720A Network Analyser and MegaPhase cables. Measurements were taken after calibrating the network analyser. The S11s were measured with the first port cable plugged in and $50 \mathrm{ohm}$ loads attached at each remaining port. The second cable was used to measure the remaining scattering parameters: the remaining ports terminated in the same $50 \mathrm{ohm}$ load.

The radiation pattern testing was preformed in the anechoic chamber at Carleton University. The setup is shown in Figure 5.4.

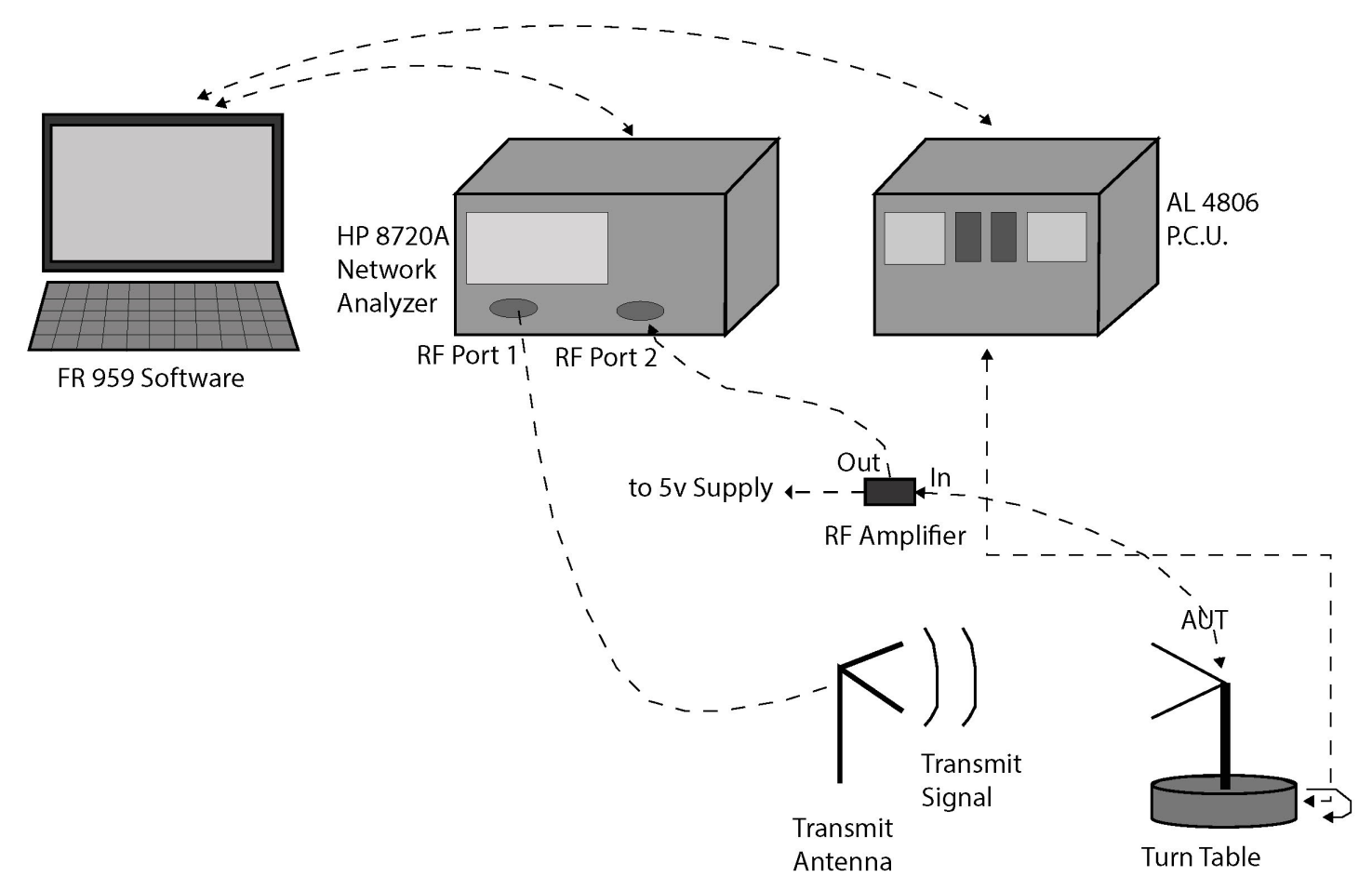

Figure 5.4: Setup of the radiation pattern testing. 


\subsection{Component Results}

The phase of each cable was measured to determine their relativity to the specifications. The results are shown in Table 5.1.

Table 5.1: Measured phases of cables.

\begin{tabular}{cc}
\hline Length (in) & Phase (Degrees) \\
\hline \hline 9.11 & 23.7 \\
12.41 & -18.4 \\
\hline
\end{tabular}

The cables phase range from being 151.6 degrees to 166.3 degrees off: drastically different then what was expected. This can be fixed in future revisions by changing the lengths of cables or the lengths of lines after the three-way divider feeding the outer patches.

Similar tests were carried out for the different power dividers in order to verify operation and determine if any changes were required. The layout of the dividers are shown in Figure 5.5 


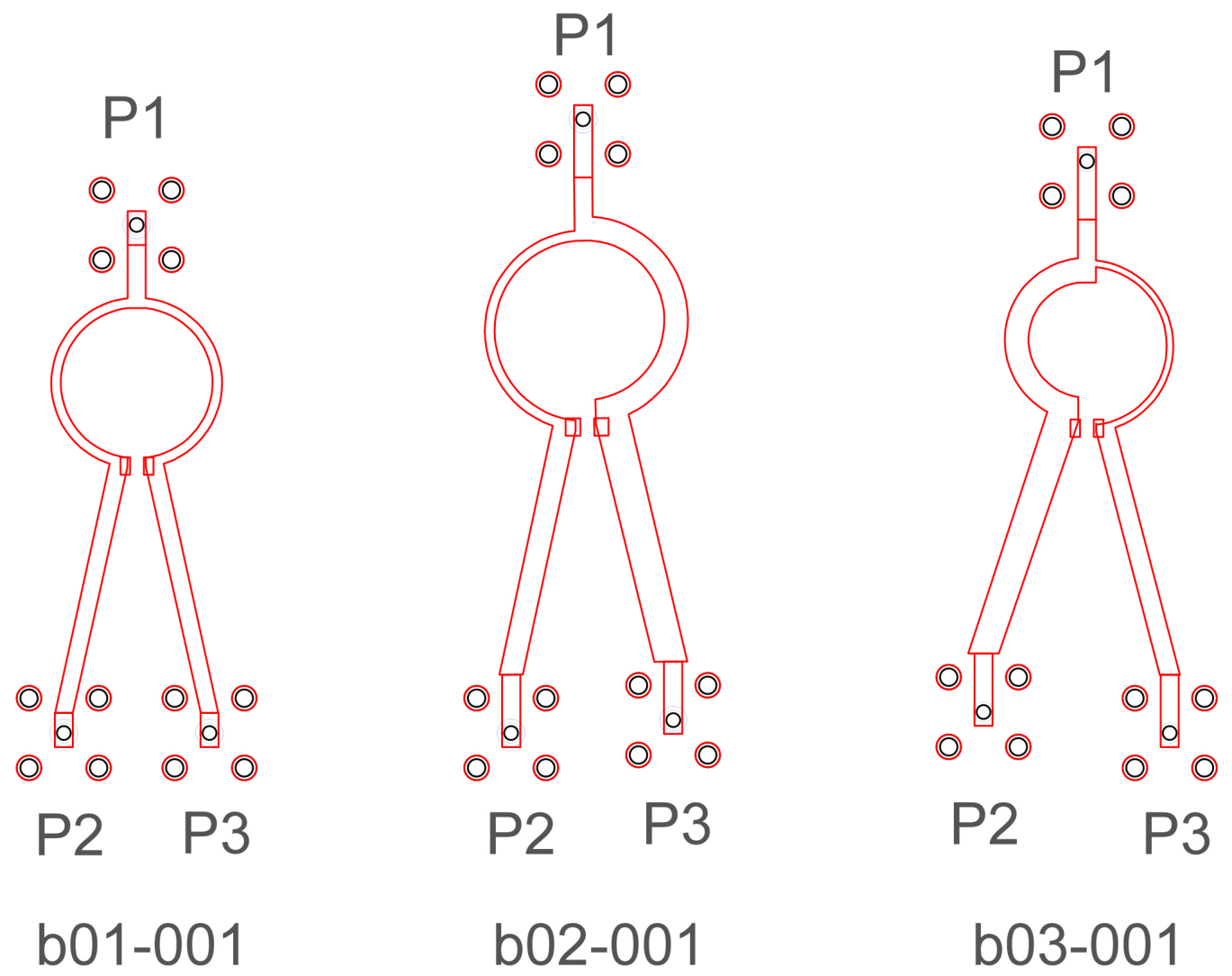

Figure 5.5: Layout of two-way dividers.

Table 5.2: Measured scattering parameters of dividers.

\begin{tabular}{ccccc}
\hline Divider & S11 & S12 & S13 & S23 \\
\hline \hline B01 (A) & -18 & -3.6 & -3.6 & -19.7 \\
B02 (B) & -21 & -2.66 & -4.8 & -20 \\
B03 (C) & -15.1 & -5.4 & -2.15 & -16 \\
\hline
\end{tabular}

The dividers could also be optimized based on the differences between manufactured and simulated. Most notably, Divider C had a nearly -2dB lower S12 from expected once manufactured. Results of three different configurations of three-way dividers in Figure 5.6 are shown in Table 5.3. 

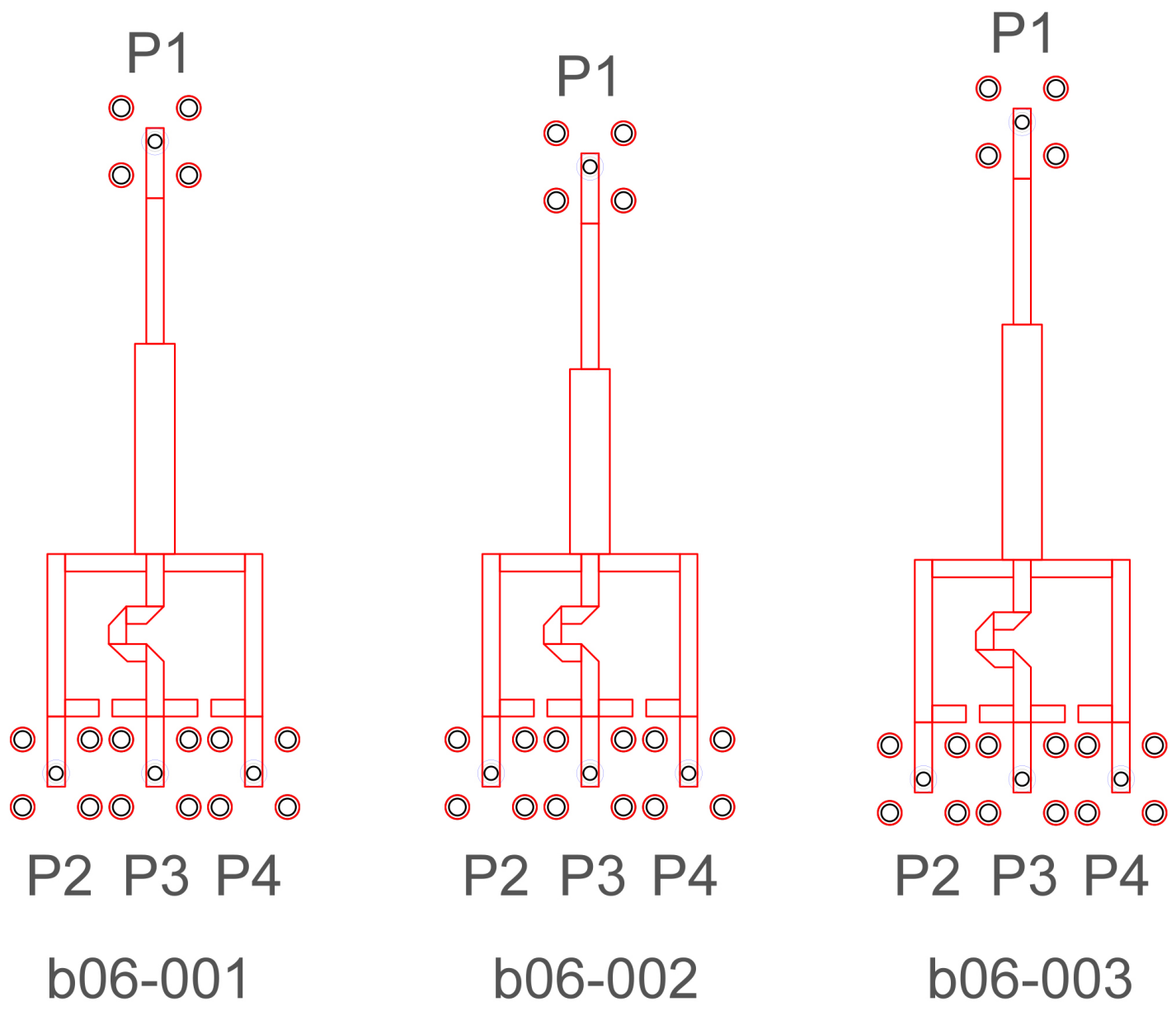

Figure 5.6: Layout of three-way dividers.

Table 5.3: Measured scattering parameters of three-way dividers.

\begin{tabular}{cccccc}
\hline Divider & S11 & S12 & S13 & S14 & S24 \\
\hline \hline B06-001 (Standard) & -19.1 & -5.15 & -5.2 & -5.55 & -12.9 \\
B06-002 (75 thou shorter $)$ & -17.8 & -5.16 & -5.12 & -5.37 & -12.1 \\
B06-003 (75 thou longer) & -18.75 & -5.26 & -5.02 & -5.1 & -15.5 \\
\hline
\end{tabular}

For the three way divider changing the length of the wider top arm had little impact on the scattering parameters of the divider. All values were at a maximum $0.35 \mathrm{~dB}$ apart. The feed network was split into smaller sections to measure the phase 
and amplitude of different sub sections. Figures 5.7 and 5.8 indicates the different sections of feed network and the port numbers assigned to them.

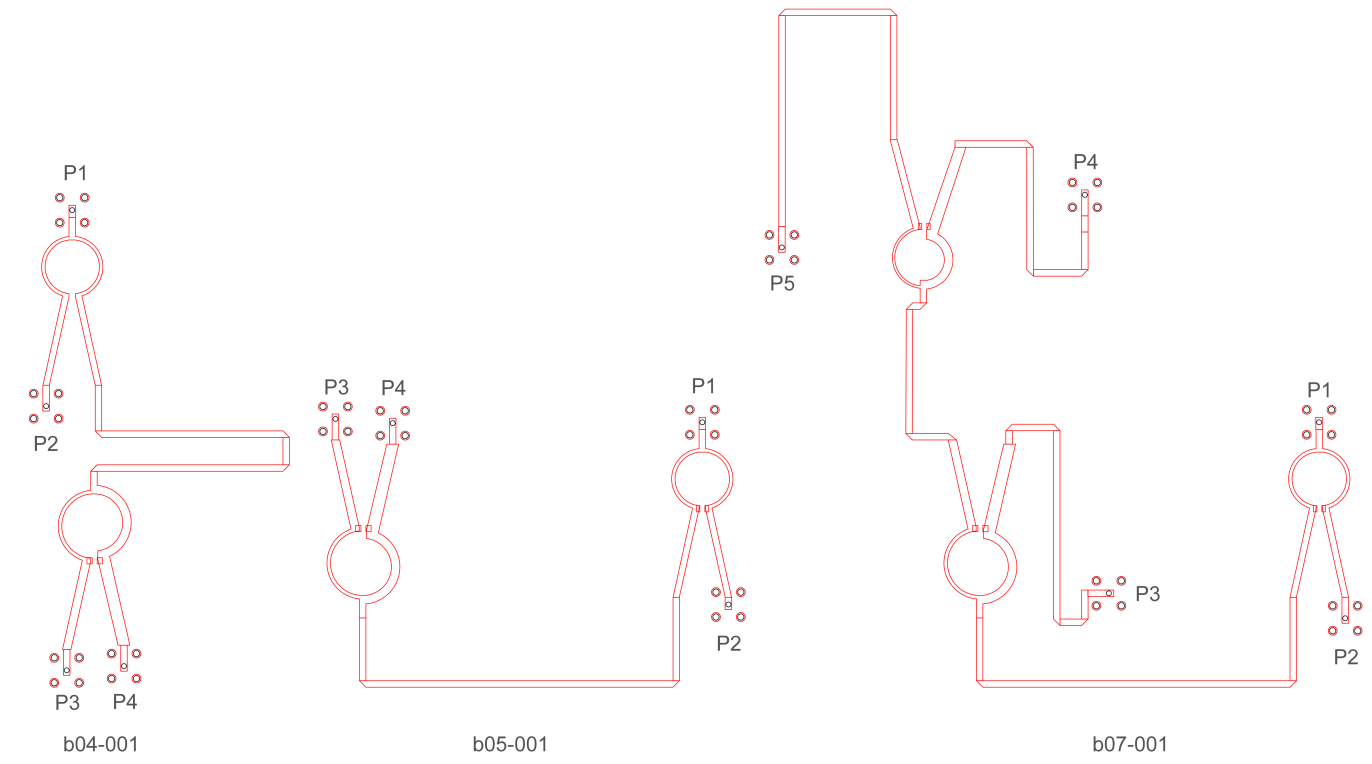

Figure 5.7: Layout of different sections of feed network. 


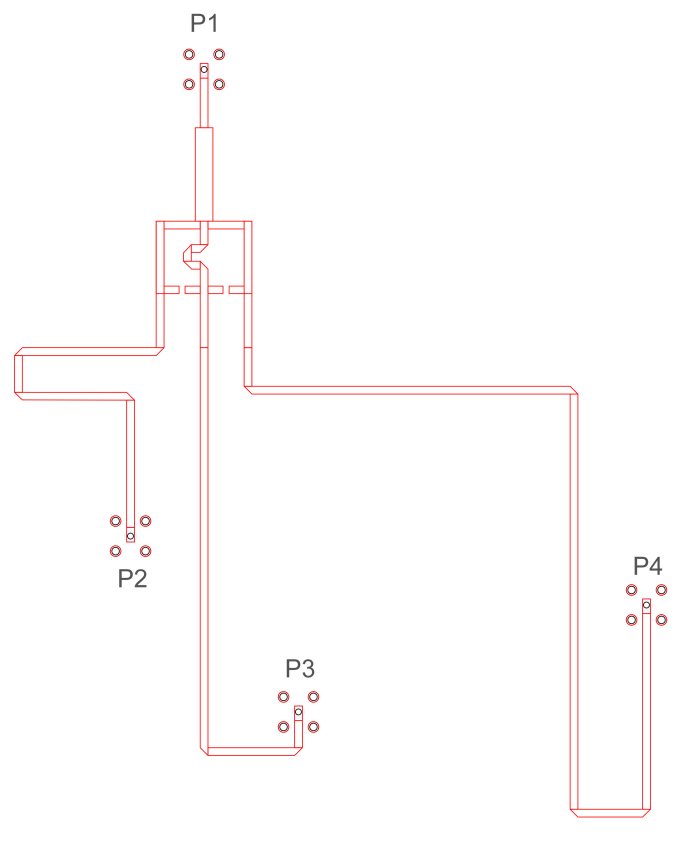

b08-001

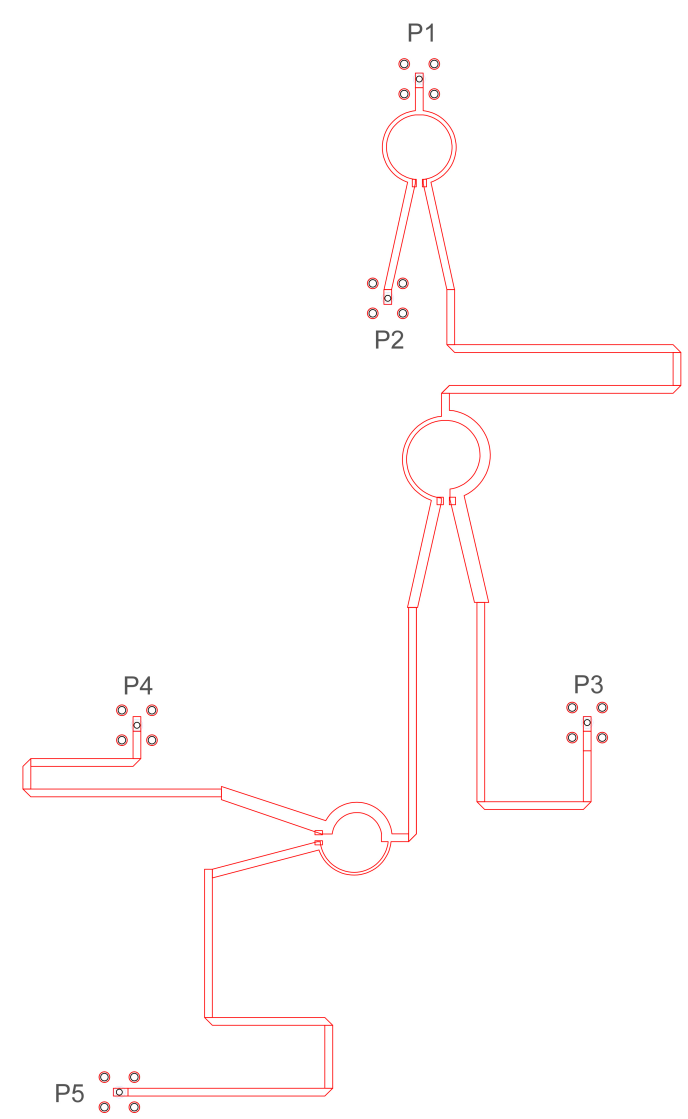

b09-001

Figure 5.8: Layout of different sections of feed network.

Table 5.4: Measured scattering parameters of feed network sections.

\begin{tabular}{cccccc}
\hline Divider & S11 & S12 & S13 & S14 & S15 \\
\hline \hline B04 & -20.3 & $-3.8 \angle 67$ & $-6.14 \angle 129$ & $-8.34 \angle 127$ & \\
B05 & -15.1 & $-3.8 \angle 81$ & $-6.13 \angle 121$ & $-8.39 \angle 120$ & \\
B07 & -23.6 & $-3.73 \angle 65$ & $-6.22 \angle 177$ & $-10.4 \angle 179$ & $-13.5 \angle 26.7$ \\
B08 & -15 & $-6.4 \angle-142$ & $-5.77<-60$ & $-5.9 \angle 25$ & \\
B09 & -38.5 & $-3.73 \angle 78$ & $-6.32 \angle-170$ & $-10.7 \angle-163$ & $-13.7 \angle 45$ \\
\hline
\end{tabular}

These results show that the first sub-section of both quarters of the feed network produce magnitudes and phases that are very similar. In sections 007 and 009 the 
magnitude and phases delivered to the patches in ports 3 and 4 - as expected. This proves that the feed network provides the correct magnitude and phase to each of the patches.

The radiation pattern of the full array with a 344 thou air gap was tested with the results in Figure 5.9, and a return loss of -15dB. A 250 thou air gap was tested with its azimuth, elevation, and the 45 degree cut radiation pattern shown in Figure 5.10. The Gaussian distribution was also tested by removing the four outer antennas and loading the ports with $50 \mathrm{ohm}$ loads (results illustrated in Figure 5.11). The return loss, bandwidth, and antenna specifications of these configurations are in Table 5.5.

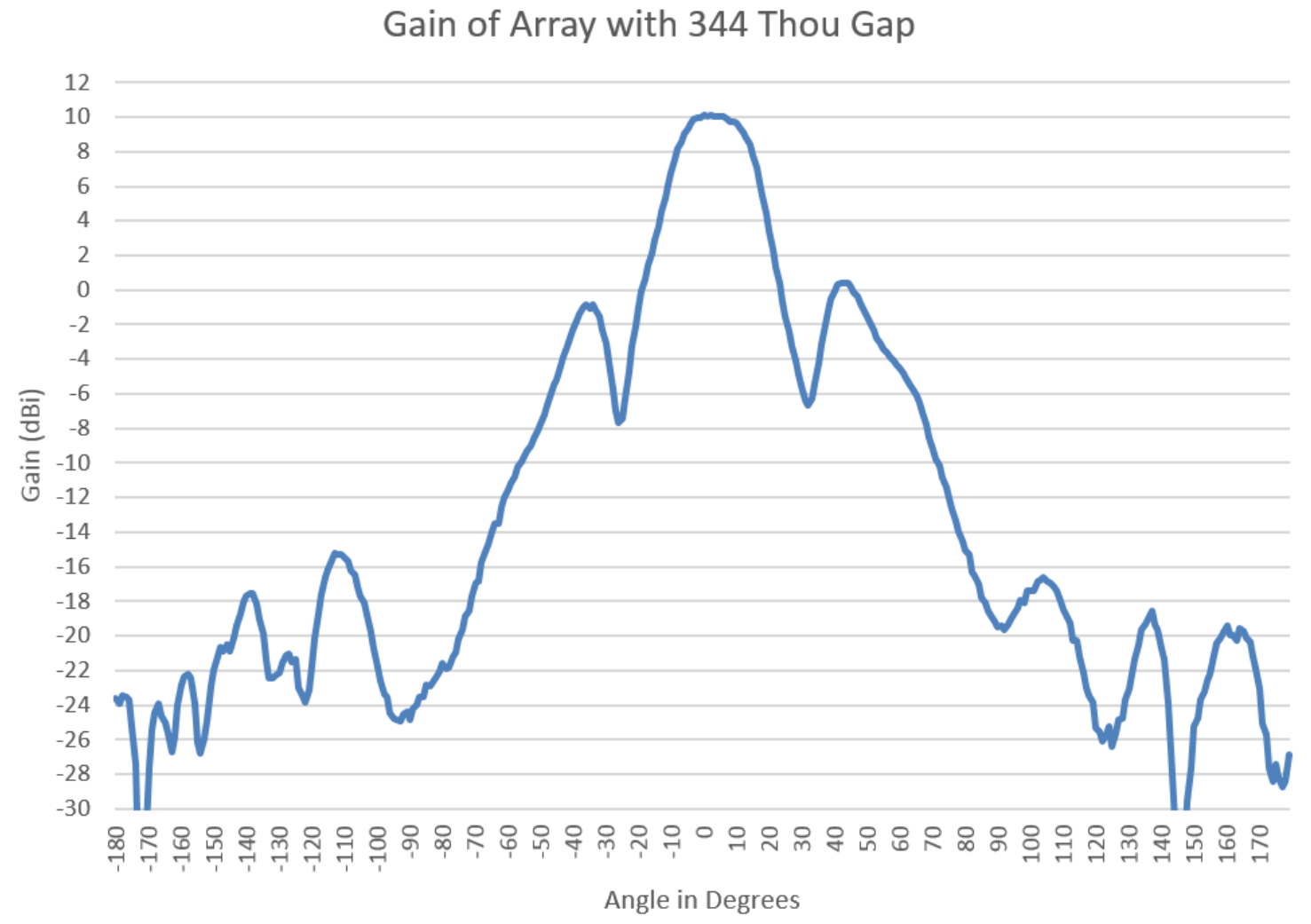

Figure 5.9: Radiation pattern of array with 344 thou air gap. 


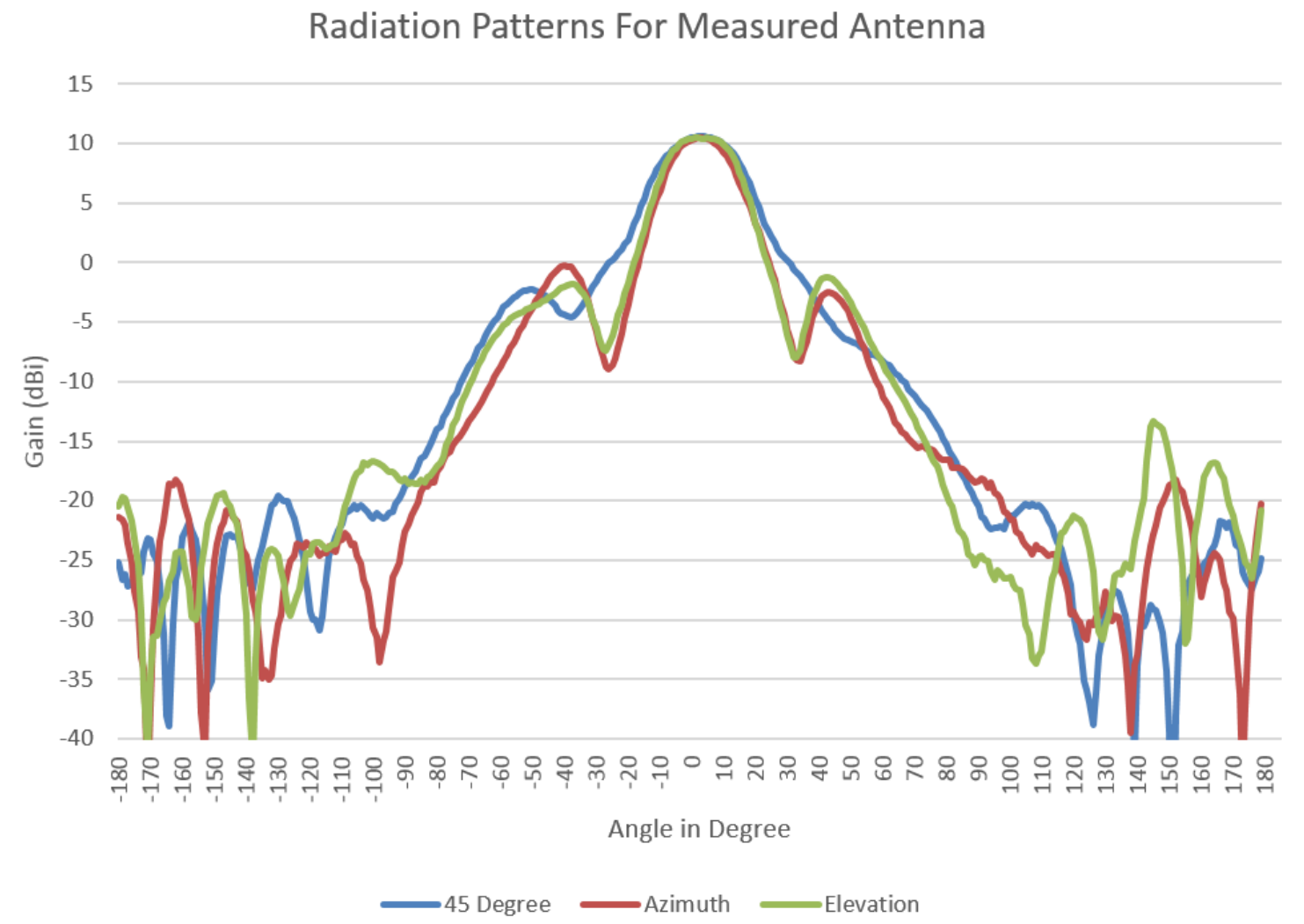

Figure 5.10: Radiation pattern of array with 250 thou air gap. 


\section{Gain of Gaussian Distribution with 344 Thou Gap}

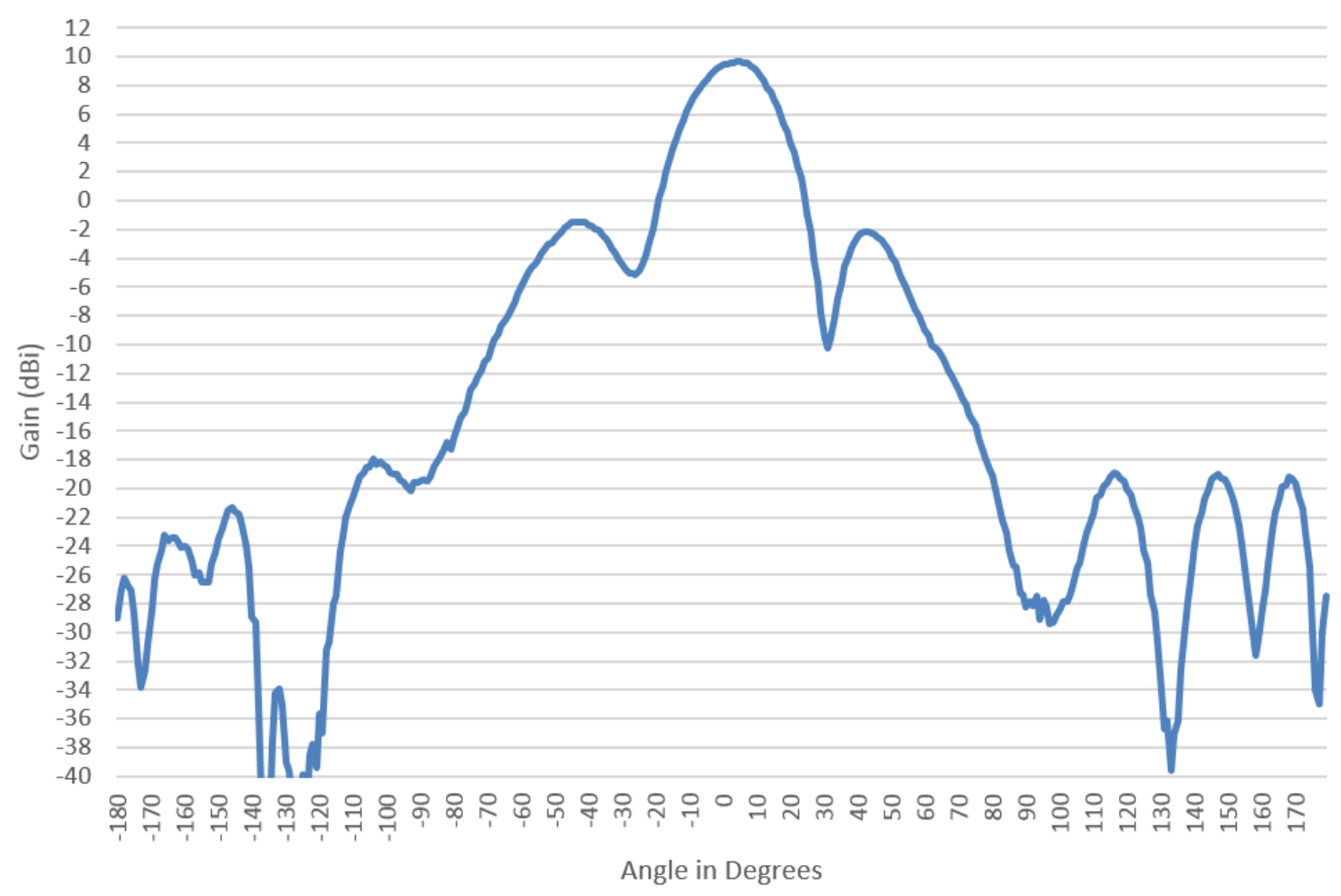

Figure 5.11: Radiation pattern of Gaussian array with 344 thou air gap.

Table 5.5: Results of full antenna configurations.

\begin{tabular}{cccccc}
\hline Antenna & S11 @ 2.45GHz & $\begin{array}{c}\text {-10dB Bandwidth } \\
\text { GHz-GHz }\end{array}$ & $\begin{array}{c}\text { Gain } \\
\text { dBi }\end{array}$ & $\begin{array}{c}\text { HPBW } \\
\text { Degree }\end{array}$ & $\begin{array}{c}\text { Max SLL } \\
\text { dB }\end{array}$ \\
\hline \hline 250 Thou Air Gap & -15 & $2.386-2.485$ & 10.5 & 24 & 11.8 \\
Gaussian & -15.4 & $2.382-2.483$ & 9.7 & 26 & 12.2 \\
344 Thou Air Gap & -15.4 & $2.382-2.483$ & 10.1 & 24 & 10.1 \\
\hline
\end{tabular}

The return losses are better then simulated. This is likely because the losses associated with the cables, as the reflected power will be less due to the losses. The air gap however did not contribute to the return loss or bandwidth. The smaller air gap has a slightly larger bandwidth as well as higher gain and lower sidelobe levels, in comparison to the larger air gap. The gain is lower then the target and directivity in 
the previous chapters simulations. These can be explained by the fact the directivity does not take into account the efficiency of the antenna. The mismatches in the feed network result in a drop in overall gain, however if the impedance mismatches were corrected, the gain would improve. As expected the Gaussian distribution has lower sidelobe levels. 


\section{Chapter 6}

\section{Conclusions}

This thesis began with the objective of improving the performance of stadium Wi-Fi installations. A square beam with low sidelobe levels would allow for less interference between adjacent sections in the stadium. First a square beam was produced using a full array. A method to develop a sparse array through the reduction of a full array, is highlighted in Section 3.4. This method uses the duality between DSP and array theory to transform a sinc weighting in the near-field to a square beam in the far-field. The elimination of low powered elements allowed for the creation of a square beam with just twenty-one patches. The sparse array allows for additional patches to be filled in. Secondly, a sub array to reactively reduce sidelobe levels of this sparse array was added with steps detailed in Section 3.6.

The third component designed was the feed network. This included the design of equal and unequal split Wilkinson power dividers. Reflections caused by mutual coupling are additive throughout the feed network making it difficult to impedance match using standard techniques. A unique method to reduce return loss and simulation time was developed in Section 4.6. Simulations were run simultaneously with the design process.

The feed network simulated in Section 4.6 shows that the phases differed with and 
without patches present. The reflections from the patches caused phases to be altered that affected matching and the radiation pattern. These mismatches are difficult to overcome because any random error in the length or thickness of the microstrip line would cause a problem in matching.

The manufactured antenna was then assembled by hand, and tested in anechoic chamber. Even though not all the original specifications were met, many were. These specifications include a square beam and appropriate return loss. The feed network did not however, provide the correct amplitude and phase to all the patches - although if more processing power was available, the network could be optimized.

Results from testing the manufactured prototype are a gain of $10.5 \mathrm{~dB}$ and a max SLL of $-11.8 \mathrm{~dB}$. A HPBW of 24 degrees and a S11 of $-15 \mathrm{~dB}$ at $2.45 \mathrm{GHz}$ with a -10dB bandwidth between $2.386 \mathrm{GHz}$ and $2.485 \mathrm{GHz}$. These specifications are shown in Figure 6.1. 


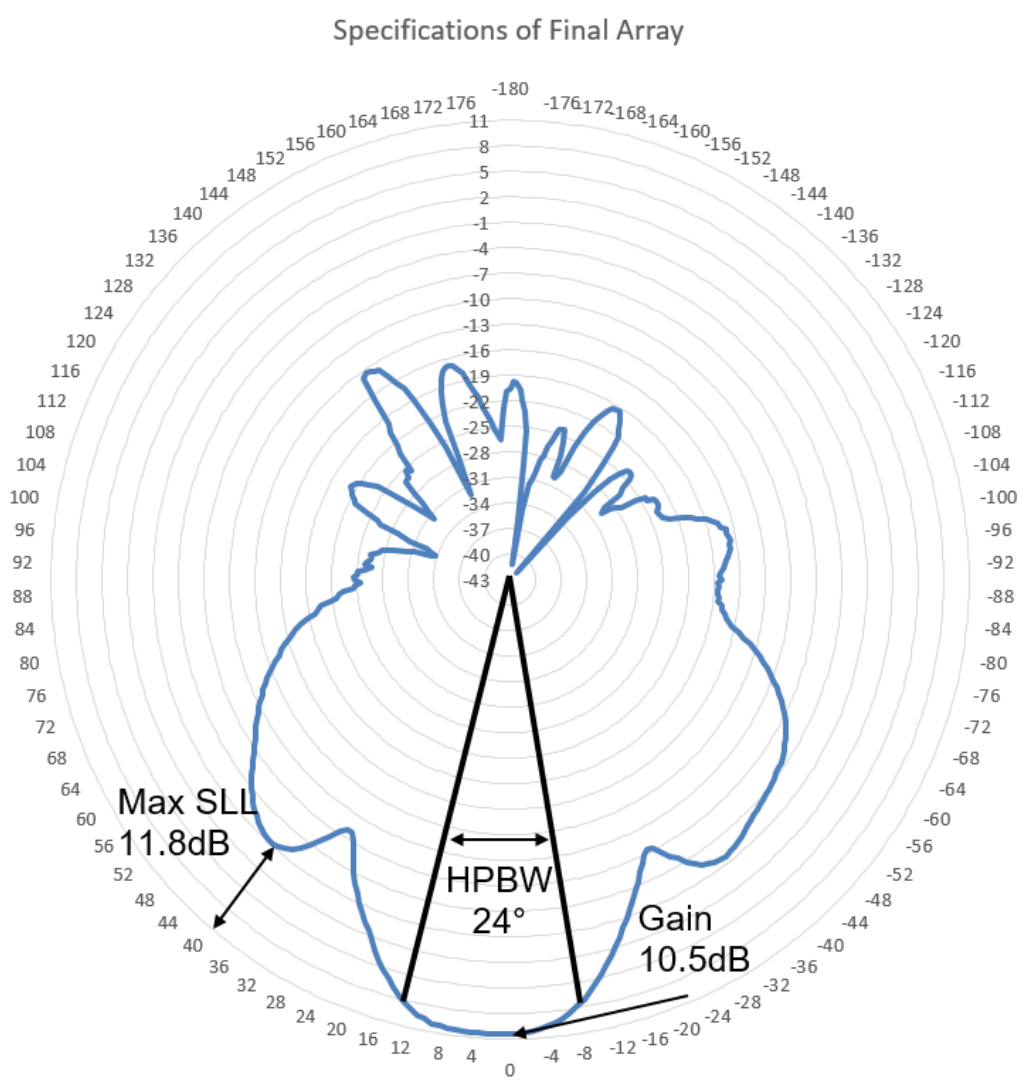

Figure 6.1: Radiation pattern with relevant specifications labeled.

The full antenna was tested and compared with the simulation results. These results are overlaid in Figure 6.2. Given the results for both the measured and simulated tests are similar, it is concluded that when simulated results were improved, the measured results would follow. In addition, this result coupled with the correlation between the feed network fed and port fed array in Section 4.8, shows that if the magnitude and phases of the feed network were optimized, the sidelobe levels would further improve. 


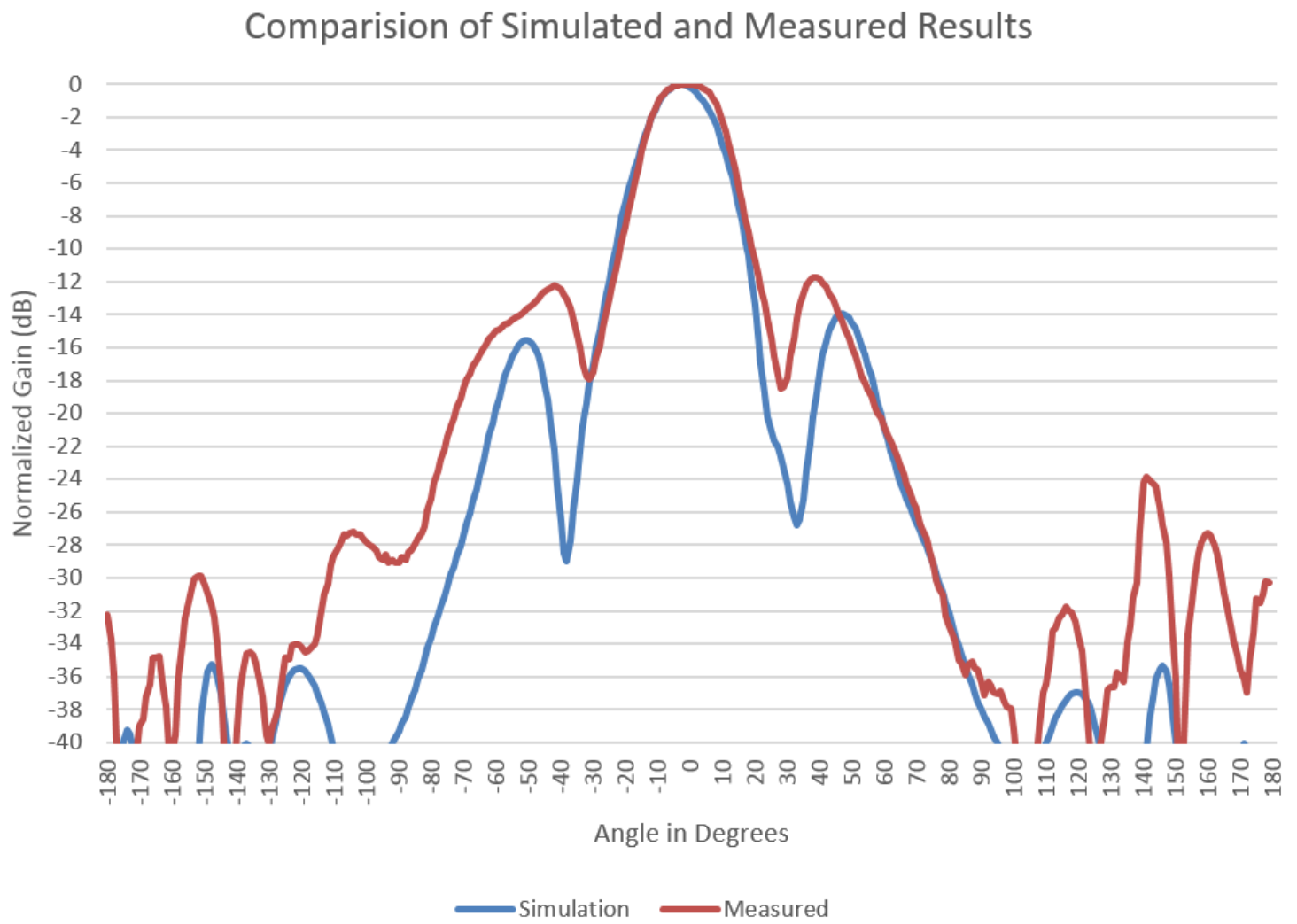

Figure 6.2: Radiation pattern of measured and simulated array.

The targets and measured values are compared in Table 6.1

Table 6.1: Target Specifications vs Measured Values.

\begin{tabular}{ccc}
\hline Specification & Target & Measured \\
\hline \hline Gain (dBi) & 18 & 10.5 \\
HPBW (Degrees) & 20 & 24 \\
Max SLL (dB) & -30 & -11.8 \\
Bandwidth (GHz-GHz) & $2.4-2.483$ & $2.386-2.485$ \\
Return Loss $(\mathrm{dB})$ & -12 & -15 \\
Size $(\mathrm{mm} \times \mathrm{mm})$ & $600 \times 600$ & $557.57 \times 557.57$ \\
\hline
\end{tabular}




\subsection{Future Work}

The primary focus of future research would be to optimize the feed network, continuing to reduce sidelobe levels. This involves adjusting the line lengths to each patch to optimize the phase delivered.

Another improvement would be to implement the optimized location of the sidelobe suppressing sub-array allowing for even lower sidelobe levels. It is also pertinent to test an array without the suppressing patches to determine the optimal location for them using the reactive method described in Section 3.6. This would account for any discrepancies between simulation and measured results. 


\section{List of References}

[1] S. Orfandidis, Electromagnetic Waves and Antennas. New Jersey: Rutgers University, 2014.

[2] A.Petosa, "Course notes: Elec 5607 - antenna engineering fall 2013."

[3] D. Pozar, Microwave Engineering, 4th ed. New York: John Wiley and Sons, 2012.

[4] Microwaves101, "Unequal-split wilkinsons - the rest of the story." http://www.microwaves101.com/encyclopedias/unequal-split-wilkinsons-therest-of-the-story, 2011. Accessed: 2015-07-14.

[5] L. B. R. Garg, P. Bhartia and A. Ittipiboon, Micrstrip Antenna Design Handbook. MA: Artech House, 2001.

[6] F. K. C. Tsao, Y. Hwang and F. Dietrich, "Aperture-coupled patch antennas with wide-bandwidth and dual-polarization capabilities," in Antennas and Propagation Society International Symposium, 1988. AP-S. Digest, pp. 936-939, 1988.

[7] S. Wentworth, Applied Electromagnetics: Early Transmission Lines Approach. New York: John Wiley and Sons, 2008.

[8] "Standard dictionary of electrical and electronics terms," 100-1988.

[9] C. Balanis, Antenna Theory, 3rd edition. New York: John Wiley and Sons, 2005.

[10] E. Weisstein, "' fourier transfrom" from mathworld - a wolfram web resource."

[11] L. Parad and R. Moynihan, "Split-tee power divider," Microwave Theory and Techniques, IEEE Transactions on, vol. 13, pp. 91-95, 1965. 
[12] S. M. G. M. A. Ahmad, A. Behera and R. Ghatak, "Synthesis of flat top power pattern in linear antenna arrays using differential evolution algorithm," International Journal of Electronics and Communication Technology, vol. 4, pp. 197-199, 2013.

[13] S. Z. Y. L. G. F. Z. Zhang, N. Liu, "Wideband circularly polarised array antenna with flat-top beam pattern," IET Microwaves, Antennas and Propagation, vol. 9, pp. 755-761, 2015.

[14] B. S. H. Zhou, Y. Huang and Q. Liu, "Design and realization of a flat-top shapedbeam antenna array," Progress in Electromagnetics Research Letters, vol. 5, pp. 159-166, 2008.

[15] A. C. A. Ahmad, T. Sinhamahapatra and G. Mahanti, "Synthesis of flat-top beam pattern with a multiple concentric circular ring array antenna," Industrial and Information Systems, 2008. ICIIS 2008. IEEE Region 10 and the Third international Conference on, pp. 1-4, 2008.

[16] P. Priyadarshini and O. Vishnu, "Sidelobe canceller system for phased array radar," Radar Symposium (IRS), 2011 Proceedings International, IEEE, pp. 855$862,2011$.

[17] R. Bracewell, The Fourier Transform and Its Applications, 3rd ed. New York: McGraw-Hill, 1999.

[18] A. M. A. Aboul-Seoud and A. Hafez, "A sidelobe level reduction (sll) for planar array antennas," in 26th NATIONAL RADIO SCIENCE CONFERENCE (NRSC2009), pp. 1-8, 2009.

[19] J. Mohammed and K. Sayidmarie, "Sidelobe cancellation for uniformly excited planar array antennas by controlling the side elements," IEEE ANTENNAS AND WIRELESS PROPAGATION LETTERS, vol. 13, pp. 987-990, 2014.

[20] D. Pozar and B. Kaufan, "Design considerations for low sidelobe microstrip arrays," IEEE Transactions on Antennas and Propagation, vol. 38, pp. 1176$1185,1990$.

[21] Microwaves101, "Three-way planar wilkinsons." http://www.microwaves101.com/encyclopedias/three-way-planar-wilkinsons, 2010. Accessed: 2015-07-14. 
[22] L. Allen and L. Diamond, "A simple model for mutual coupling effects on patterns of unequally spaced arrays," Antennas and Propagation, IEEE Transactions on, vol. 15, pp. 530-533, 1967.

[23] J. Carr, Practical Antenna Handbook, 4th edition. New York: McGraw-Hill, 2001. 


\section{Appendix A}

\section{Matlab Code}

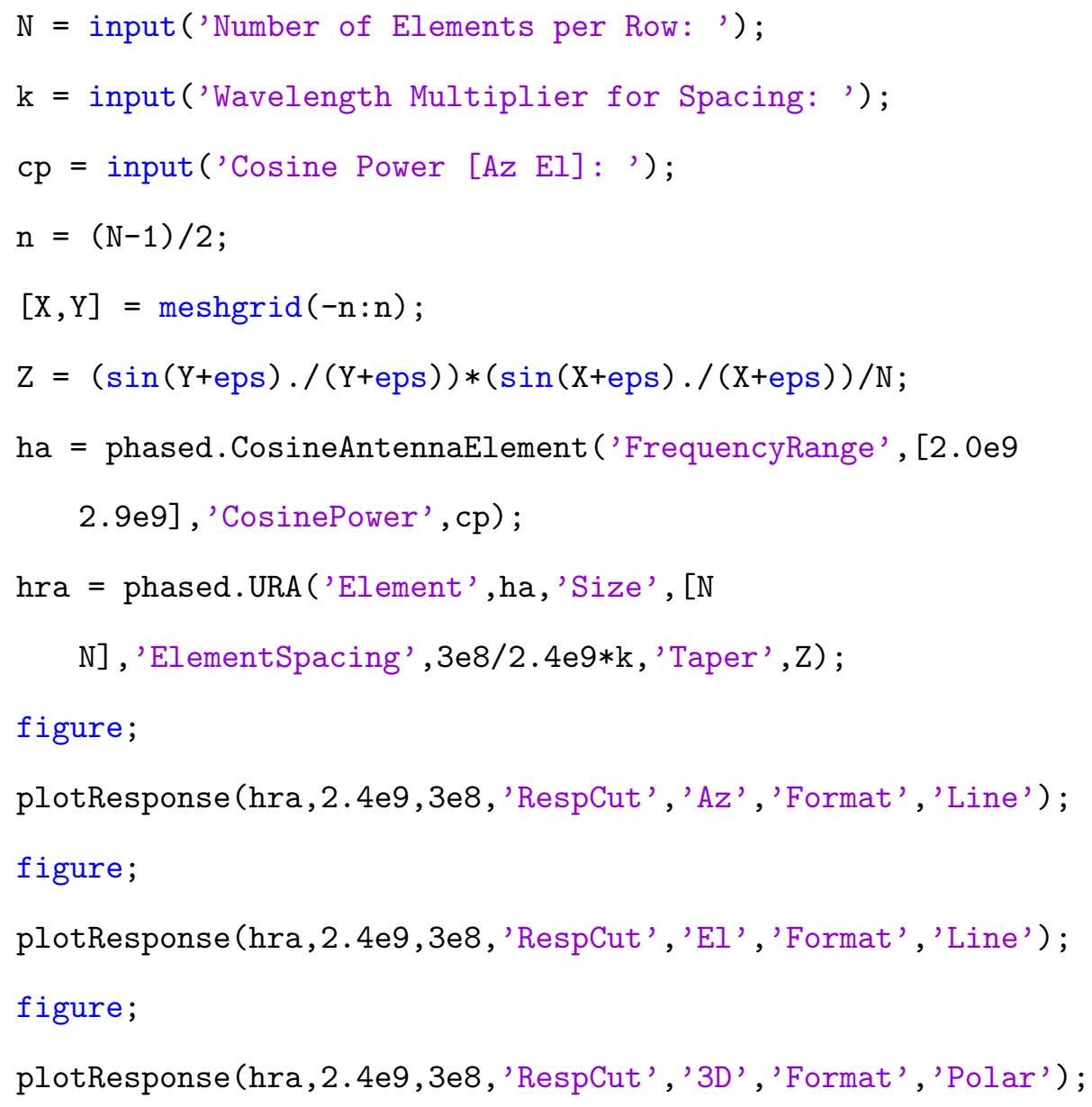

Using the phased array toolkit the above code generates the azimuth, elevation, 
and 3D plot of an array is generated. The code prompts for the number of elements per row. This will produce a square array with equal elements in the rows and columns. The next prompt is the spacing between elements in wavelengths. By default the code is written for $2.4 \mathrm{GHz}$, but the frequency can be changed by modifying any instance of ' $2.4 \mathrm{e} 9$ '. The next prompt for the Cosine power to adjust for differences in the patches radiation pattern. Typical value used was $\left[\begin{array}{ll}1 & 1\end{array}\right]$.

The variable $\mathrm{Z}$ is used for weighting the elements, in this case it is a sinc function. However, it can be any function desired. 


\section{Appendix B}

\section{Empire Setup}

Once starting Empire XPU, a prompt to start a new project will appear as shown in

Figure B.1. Selecting templates/phased array will provide the selections shown.

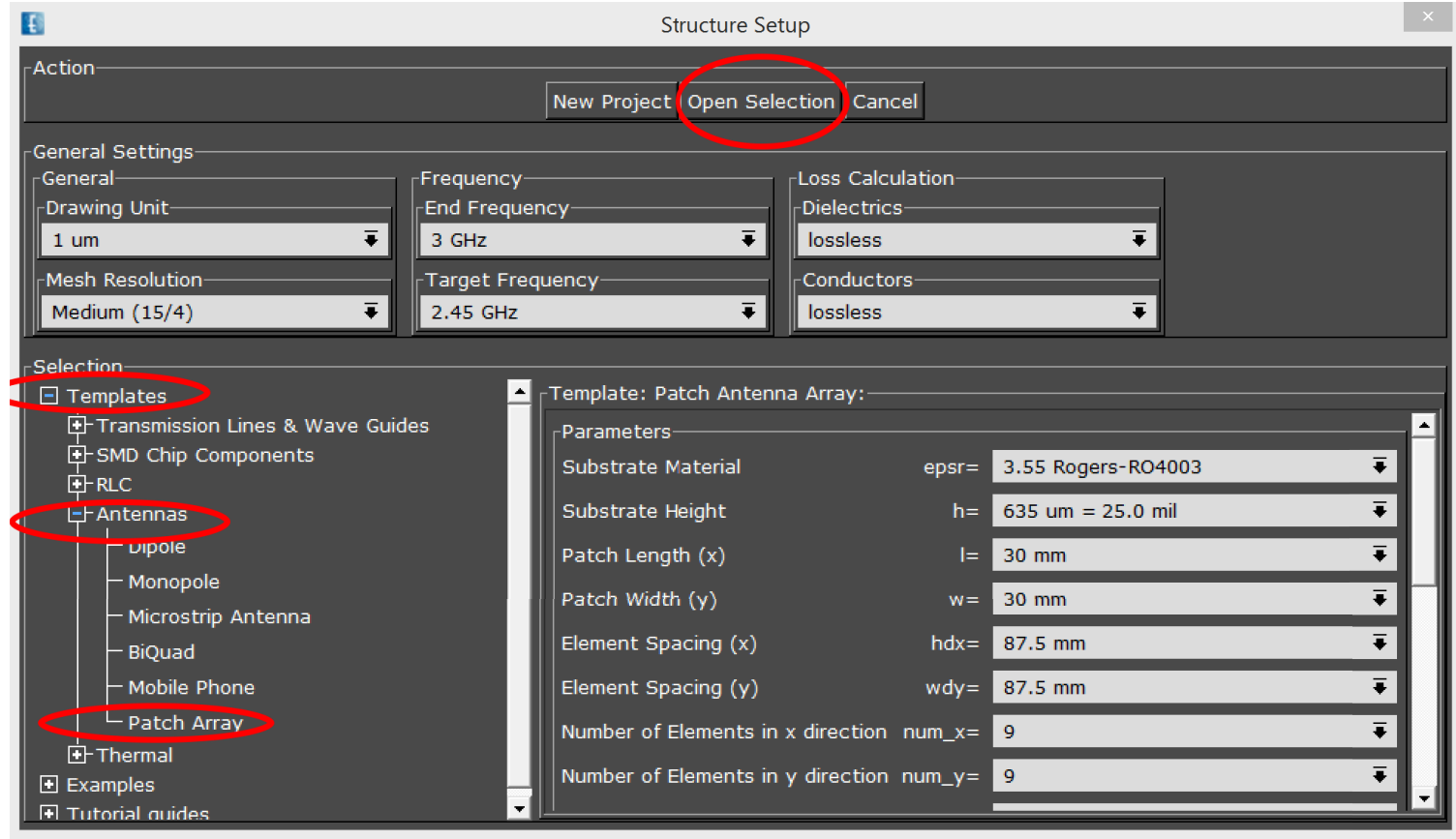

Figure B.1: Structure setup in Empire XPU.

From here, there are a number of different parameters to configure including: centre frequency, patch dimensions, element spacing, and number of elements. Once values are imputed the array will be generated by clicking "Open Selection". The 
screen in Figure B.2 is the main work screen and shows the groups. More groups can be added or removed if need be. A farfield monitor will be added by default more monitors can be added from here.

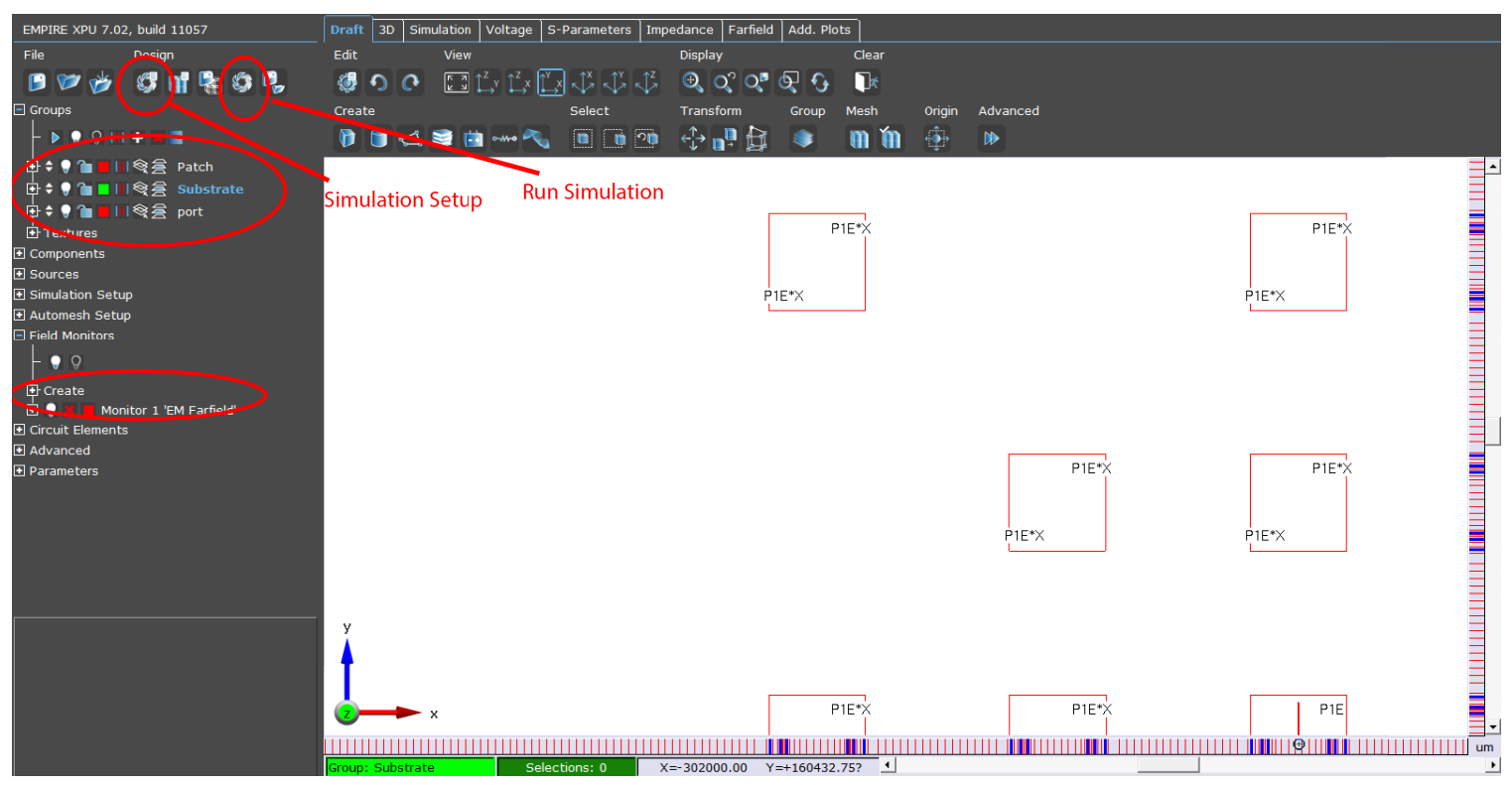

Figure B.2: Main work screen in Empire XPU.

Simulation parameters can be adjusted. By clicking the simulation settings button shown in Figure B.2, the screen in Figure B.3 appears. 


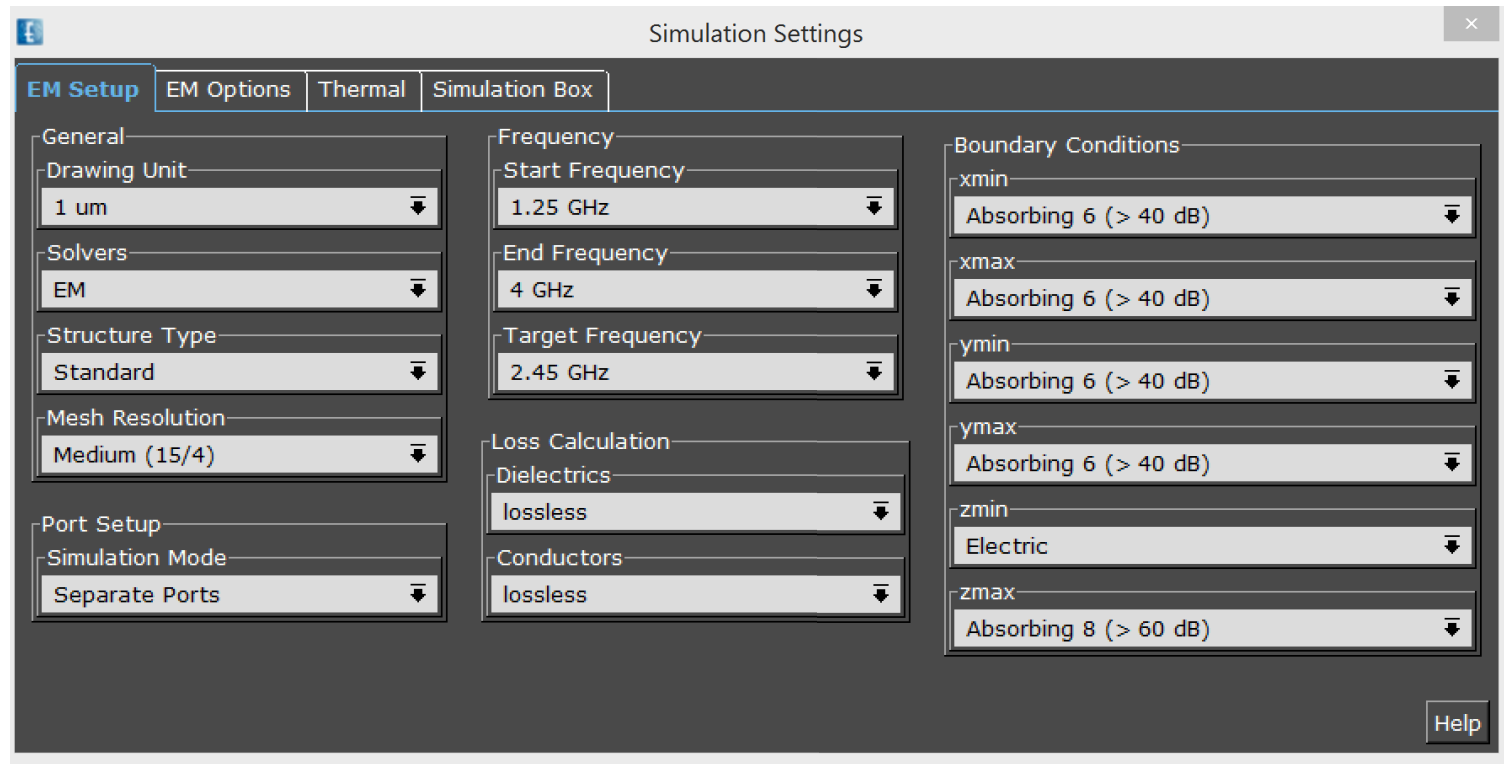

Figure B.3: Simulation Set up in Empire XPU.

Some of the settings include the mesh resolution. The finer details in the layout, the finer the mesh is required. The thin rings in the power dividers required a finer mesh. Simulation mode changes how each port is excited - one at a time or all at once. The setting for one at a time is called separate ports, and was used for the majority of the simulations. Active impedance simulates all different ports at the same time. This setting is useful to determine mutual coupling between patches or to separate sections of the array and simulate them at the same time. Different levels of loss can be used depending on the order of accuracy required. Boundary conditions define the box in which the simulation takes place. The higher the absorbing level, the longer the simulation and the more similar it is to an anechoic chamber. For zmin, electric is used because an infinite ground plan can be used for these low order simulations.

Weightings can be edited by right clicking the port on the patch and selecting Advanced Parameters, opening the menu in Figure B.4. The Excitation Current $(A)$ is what is used to set the weighting. Excitation Delay sets the phase offset, which 
is done in the form of Angle@Frequency, ie 180@2.45e9 is a 180 degree phase at 2.45 $\mathrm{GHz}$.

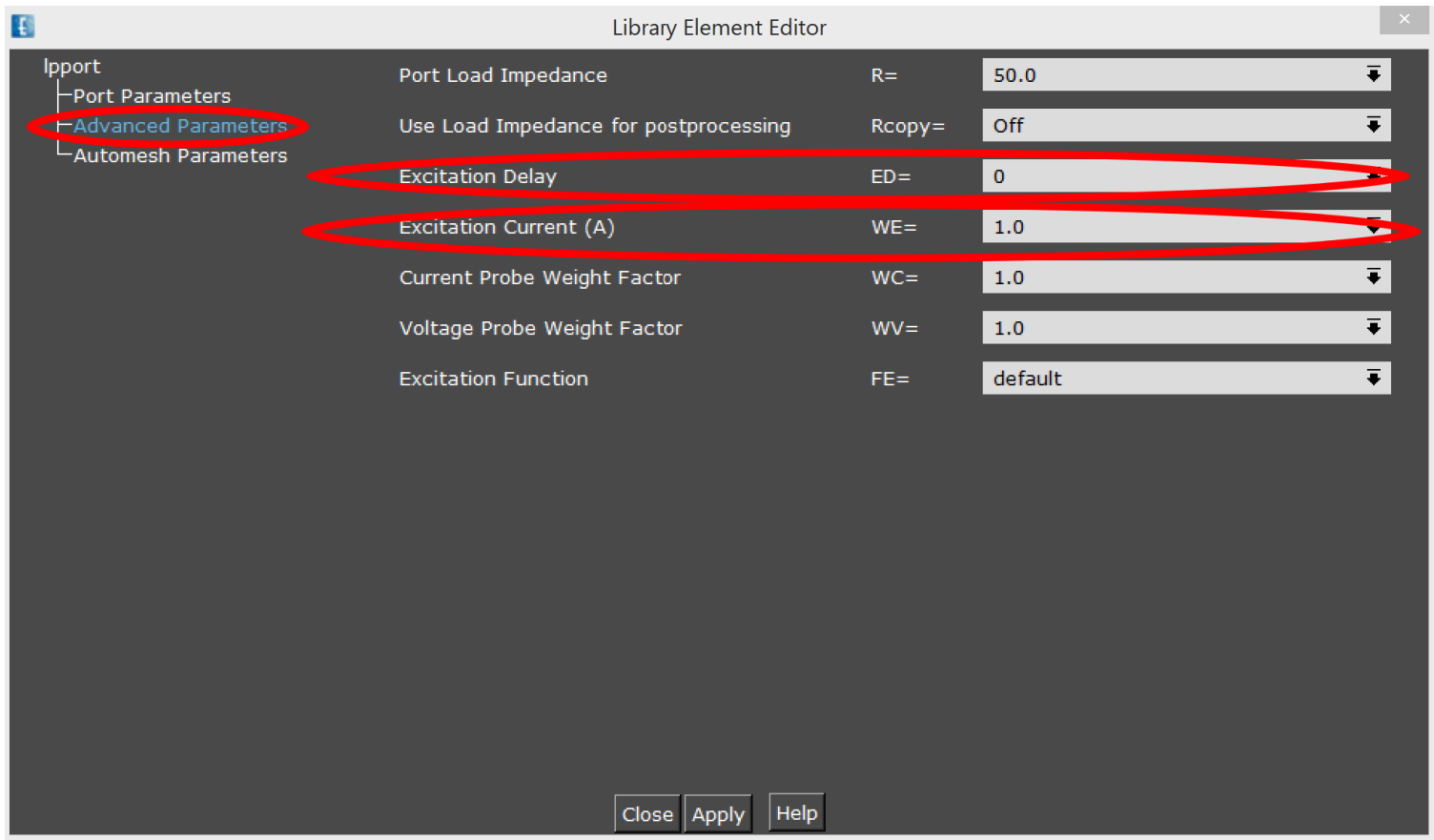

Figure B.4: Port set up in Empire XPU.

Once the weighting is set, the simulation can begin by pressing the run simulation button. After the simulation is complete the return loss is displayed and radiation pattern can be viewed by selecting the Farfield tab.

The setting initial settings used in 3 are displayed in the above figures. 